\title{
Investigation of Plasma Exhaust Profile Manipulation Using Magnetic Fields
}

\author{
Bryan A. Shambaugh
}

Follow this and additional works at: https://researchrepository.wvu.edu/etd

\section{Recommended Citation}

Shambaugh, Bryan A., "Investigation of Plasma Exhaust Profile Manipulation Using Magnetic Fields" (2017). Graduate Theses, Dissertations, and Problem Reports. 6612.

https://researchrepository.wvu.edu/etd/6612

This Thesis is protected by copyright and/or related rights. It has been brought to you by the The Research Repository @ WVU with permission from the rights-holder(s). You are free to use this Thesis in any way that is permitted by the copyright and related rights legislation that applies to your use. For other uses you must obtain permission from the rights-holder(s) directly, unless additional rights are indicated by a Creative Commons license in the record and/ or on the work itself. This Thesis has been accepted for inclusion in WVU Graduate Theses, Dissertations, and Problem Reports collection by an authorized administrator of The Research Repository @ WVU. For more information, please contact researchrepository@mail.wvu.edu. 


\title{
Investigation of Plasma Exhaust Profile Manipulation Using Magnetic Fields
}

\author{
Bryan A. Shambaugh \\ Thesis submitted to the \\ Benjamin Statler College of Engineering and Mineral Resources \\ at West Virginia University \\ in Partial Fulfillment of the Requirements \\ for the degree of
}

Master of Science

in

Aerospace Engineering

Patrick Browning, Ph.D.

V'yacheslav Akkerman, Ph.D.

Gregory Thompson, Ph.D.

Department of Mechanical and Aerospace Engineering

Morgantown, West Virginia

2017

Keywords: Magnetoplasmadynamics, Exhaust Profile Manipulation, Hypersonic Arc Jet Wind Tunnel

Copyright 2017 Bryan A. Shambaugh 


\title{
ABSTRACT \\ Investigation of Plasma Exhaust Profile Manipulation Using Magnetic Fields
}

\author{
Bryan A. Shambaugh
}

Electric propulsion systems are known for having a high specific impulse but very low thrust. In the case of a hypersonic, compressible flow, altering the exhaust profile has the potential to alter the exhaust velocity thus changing the total thrust output. In this research, the magnetoplasmadynamic (MPD) effects of applying a toroidal magnetic field to an ionized exhaust plume were investigated to manipulate the exhaust profile of the plasma jet under near vacuum conditions. The project also endeavored to determine the efficacy of permanent magnets as a replacement for electromagnetic systems in rocket propulsion.

Tests for this experiment were conducted using the West Virginia University (WVU) Hypersonic Arc Jet Wind Tunnel, in which a series of N52 grade neodymium magnets were placed in different orientations around a steel toroid mounted around the arc jet plume. Four different magnet orientations which produced different magnetic fields around the plume were tested in this experiment: two of the configurations held the magnetic poles aligned with the flow direction with north or south facing the nozzle; two configurations held the magnetic poles in a tangential orientation to the flow with north or south facing in a clockwise direction. All magnets in each of the magnetized cases were positioned equidistant to one another and equidistant to the toroid's central axis. Two additional configurations were run as control tests without any imposed magnetic fields surrounding the plume. Each test was documented using 12 sets of photographs taken from a fixed position with respect to the flow, and the photographic data was analyzed by comparing images of the exhaust plume taken 10,20, and 30 seconds after the plasma jet was activated.

Analysis of the collected images showed that the tangential configurations where the magnets' north poles were oriented clockwise held the most influence in decreasing the size of the exhaust profile across all time steps. In contrast, the axial configurations showed the highest propensity of expanding the exhaust profile across all time steps. A weight and cost analysis between permanent magnets and an electro magnet of equivalent size and field strength revealed that the additional power source required to support the electromagnet would make it heavier and less cost effective for long term missions. This study has shown that a toroidal magnetic field produced by permanent magnets can indeed produce a significant alteration in the exhaust profile of a hypersonic, compressible plasma flow. 


\section{Dedication}

I would like to dedicate this thesis to my grandfather, Walter Burd. This man, who is partly responsible for my love of space, inspires me every day to follow my dreams and become a better engineer. It gives me great pride knowing that, as an engineer, I am following in his footsteps. 


\section{Acknowledgements}

I would first like to gratefully acknowledge:

The NASA West Virginia Space Grand Consortium for their financial support in the form of a graduate research fellowship.

My thesis advisor and committee chair, Dr. Patrick Browning of the Statler College of Engineering and Mineral Resources at West Virginia University, for his continuous guidance and for providing opportunities necessary for my success.

My committee members, Dr. Gregory Thompson and Dr. Vyacheslav Akkerman of the Statler College of Engineering and Mineral Resources at West Virginia University, for their input and guidance.

Mr. Clayton Clouse for his assistance in wind tunnel diagnostics and support collecting experimental data.

Ms. Jaya Karlapati for her assistance during equipment and sensor calibrations.

Finally, I must express my profound gratitude toward my friends and family. Without their unfailing support and continuous encouragement throughout my years of study this accomplishment would not have been possible. 


\section{Table of Contents}

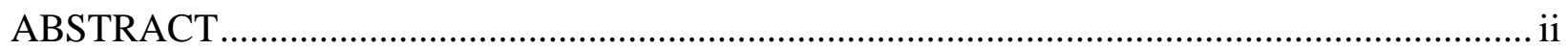

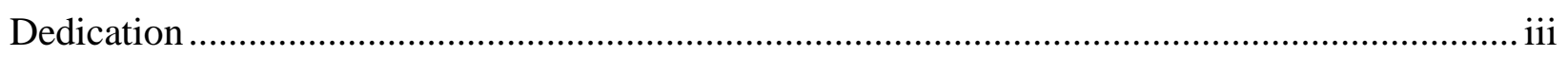

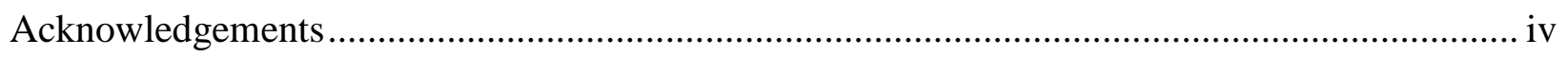

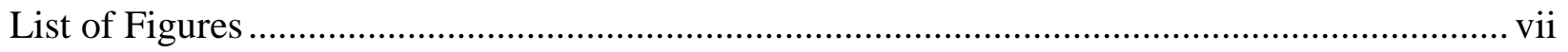

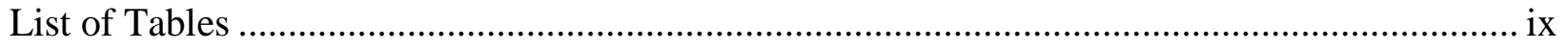

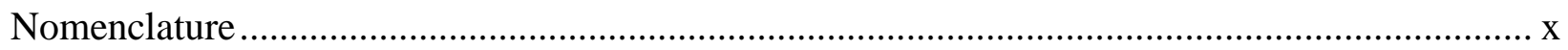

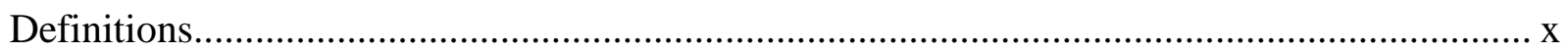

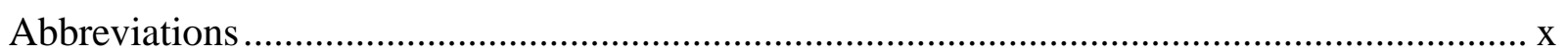

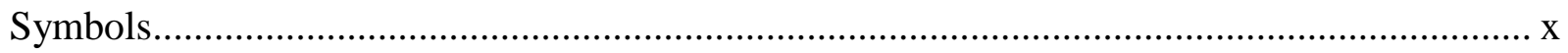

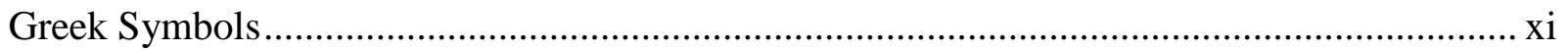

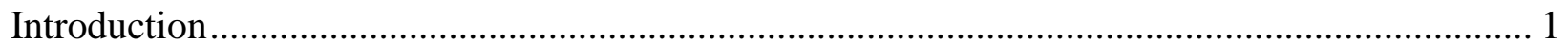

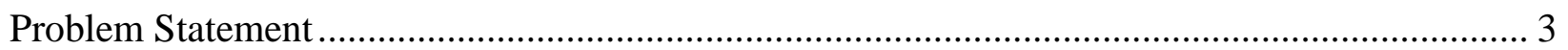

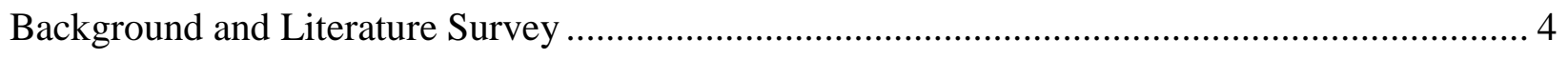

History of Electric Propulsion .................................................................................. 4

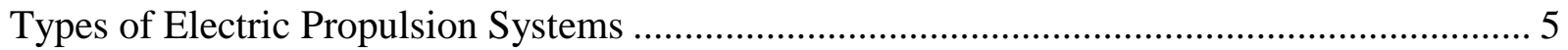

Magnetoplasmadynamics and Magnetic Nozzles ....................................................... 6

WVU Hypersonic Arc Jet Wind Tunnel ....................................................................... 8

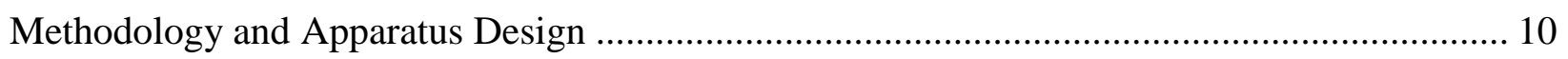

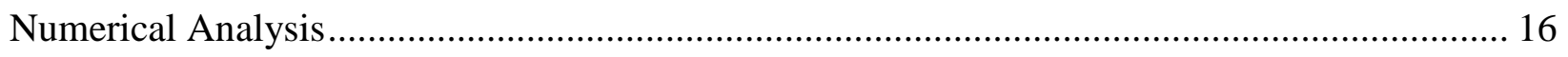

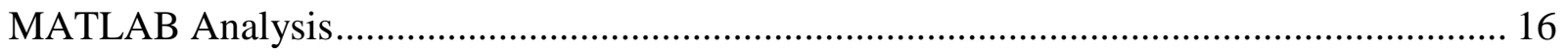

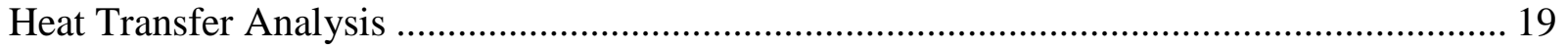

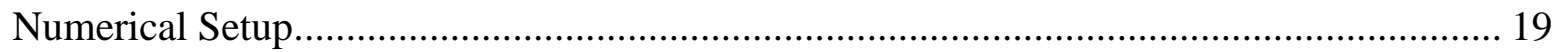

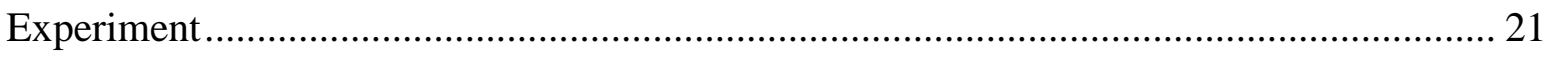

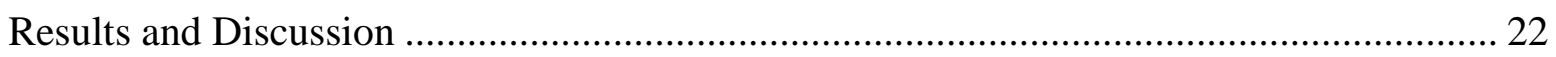

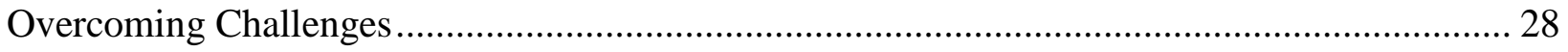

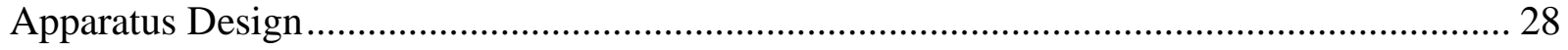

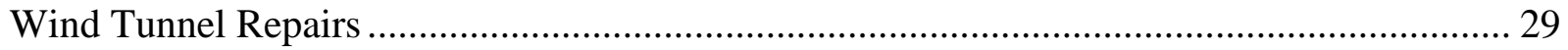

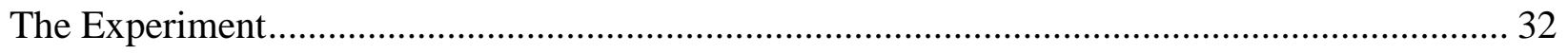

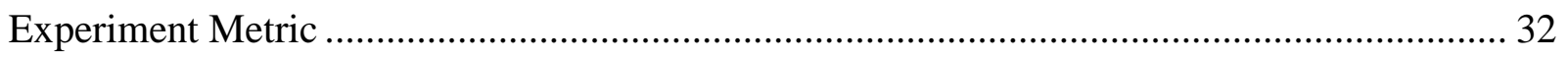

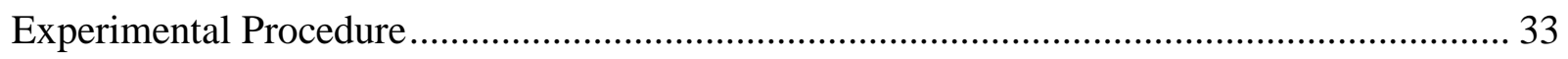




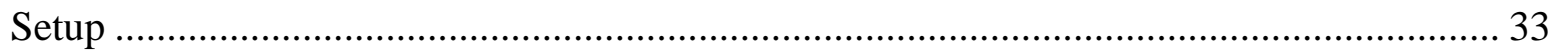

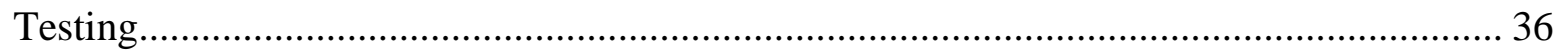

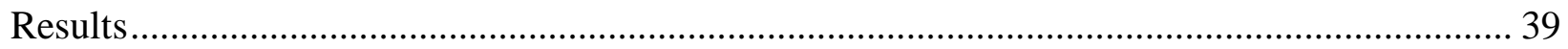

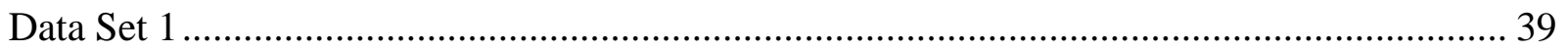

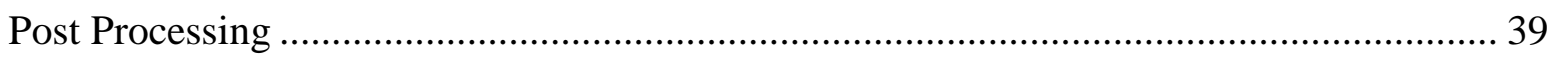

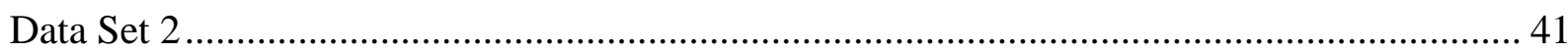

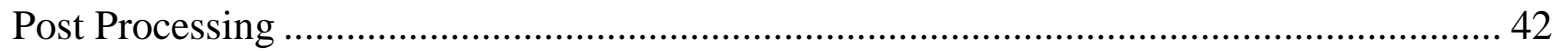

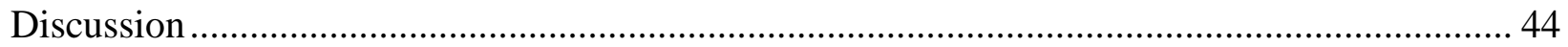

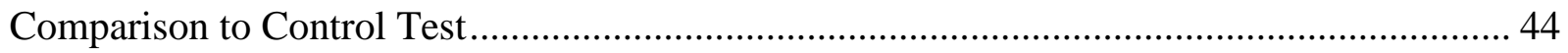

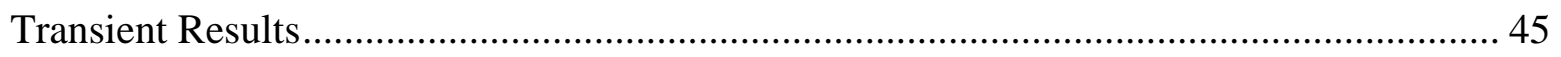

Orientation Comparisons at Each Time Step................................................................. 46

Comparison with Toroid Test ............................................................................................ 46

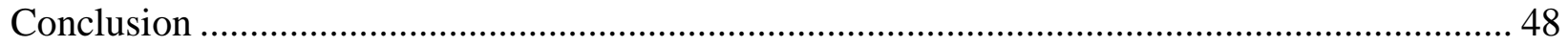

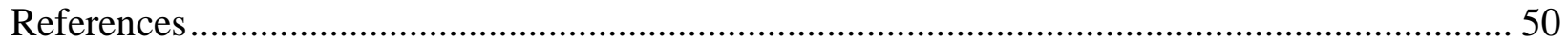

Appendix A: MATLAB Code Calculating Magnetic Field Strength ......................................... 52

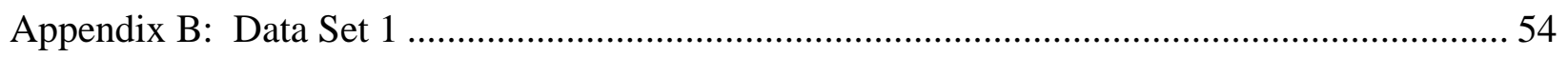

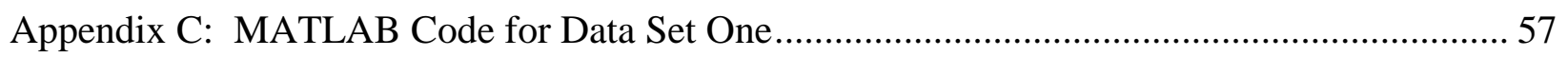

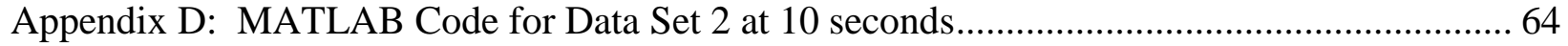

Appendix E: MATLAB Code for Data Set 2 at 20 seconds ..................................................... 72

Appendix F: MATLAB Code for Data Set 2 at 30 seconds ................................................... 80

Appendix G: Data Set 2 - Comparison to Control Test .......................................................... 88

Appendix H: Data Set 2 - Comparison to Toroid Test................................................................... 92 


\section{List of Figures}

Figure 1: Argon plasma jet ............................................................................................. 1

Figure 2: "Operating principles of a) resistojets, b) arcjets, c) Hall thrusters, d) ion engines, e) pulsed plasma thrusters, f ) field-effect electrostatic propulsion thrusters, and g) self-field

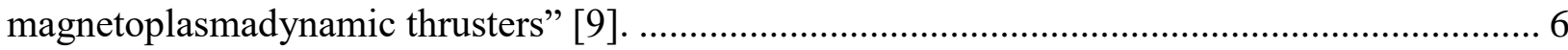

Figure 3: "Specific impulse and thrust comparison for space propulsion methods" [5] ............... 7

Figure 4: Dismantled arc jet assembly with (a) cathode and (b) anode....................................... 9

Figure 5: Cutaway CAD view of the WVU Arc Jet Hypersonic Wind Tunnel............................. 9

Figure 6: Full scale CAD model of the test apparatus ................................................................ 11

Figure 7: Unmodified shopping cart wheel from ACE Hardware [18] ...................................... 12

Figure 8: Toroid mounted in wind tunnel .............................................................................. 13

Figure 9: Movement of charged particles based on a 0.7343 Tesla magnetic field..................... 16

Figure 10: Decay of a magnetic field [Tesla] as distance [m] from the magnet's central axis

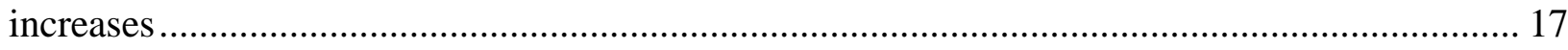

Figure 11: Magnetic field lines for a single magnet in free space [6] ................................... 18

Figure 12: Magnetic field lines through a toroidal structure [6] ............................................... 19

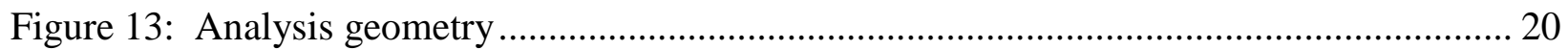

Figure 14: The instrumented toroid model mounted in the wind tunnel with positions shown for

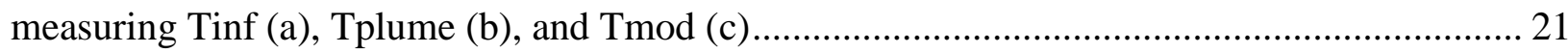

Figure 15: "The emissivity of an argon plasma at $\mathrm{T}=12000 \mathrm{~K}$ " [20] …………………….......... 22

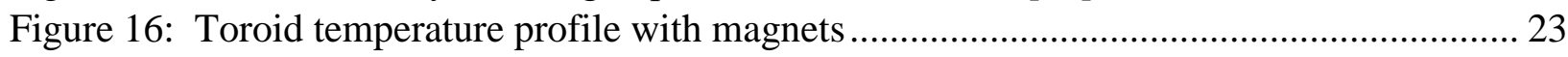

Figure 17: Toroid temperature profile without magnets .......................................................... 23

Figure 18: Magnet mounting positions assuming perfect abutment ............................................ 24

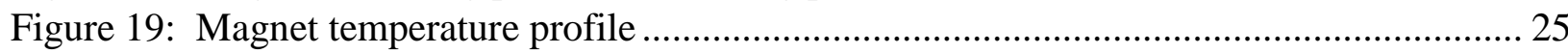

Figure 20: Time-variant experimental measurement of the plume temperature......................... 26

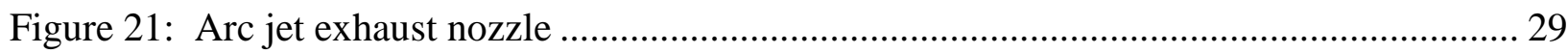

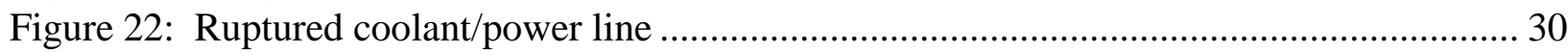

Figure 23: Repaired coolant/power line............................................................................. 31

Figure 24: Wind tunnel control console with (a) vacuum pressure gauge, (b) injector pressure gauge, (c) flow meter, (d) ammeter, and (e) volt meter ............................................................ 32

Figure 25: Multichannel thermometer connected to thermocouples located inside the vacuum

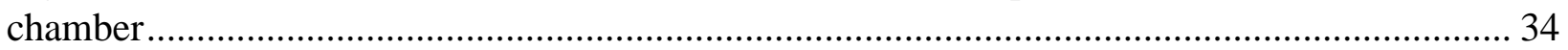

Figure 26: Pressure gauge connected to the argon cylinder ....................................................... 34

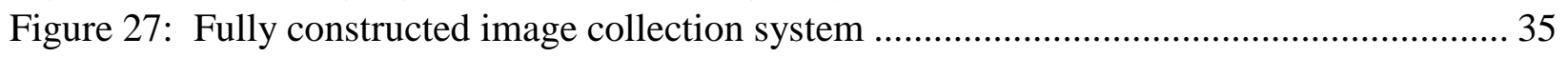

Figure 28: Apparatus used to focus the camera before data collection ………………………..... 36

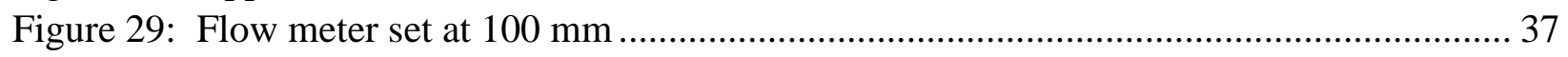

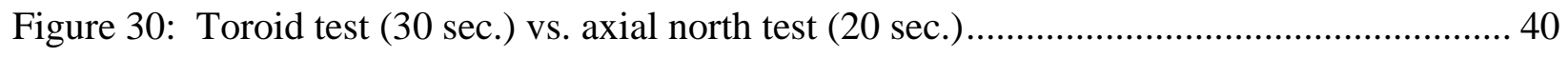

Figure 31: Axial north test $(20 \mathrm{sec}$.) vs. axial south test $(30 \mathrm{sec}$.) ……........................................ 40

Figure 32: Toroid test $(10 \mathrm{sec}$.$) vs. axial south test (30 \mathrm{sec}$.$) ...................................................... 41$

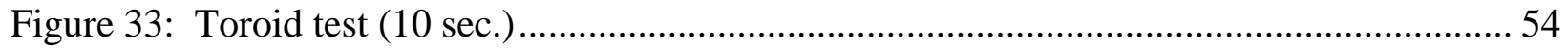

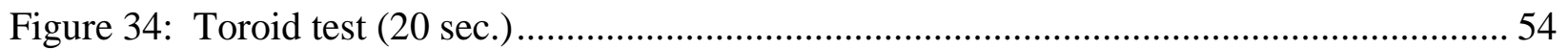

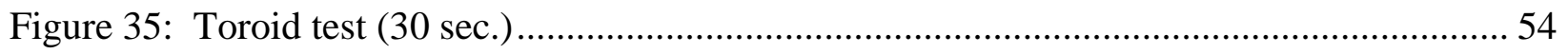




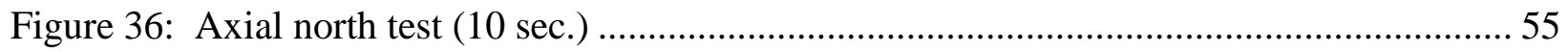

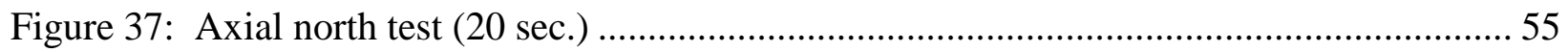

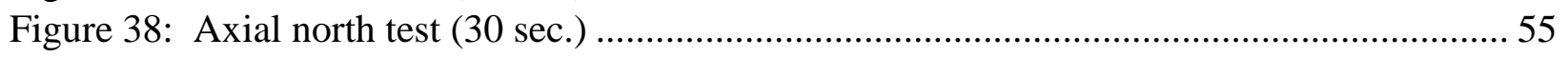

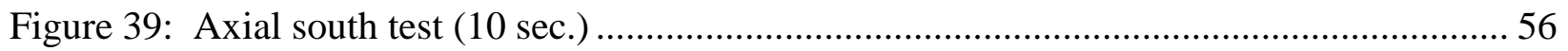

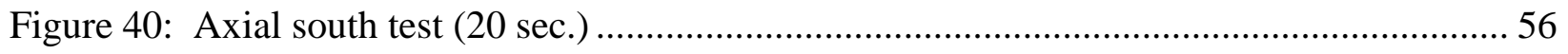

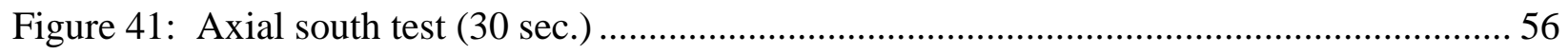

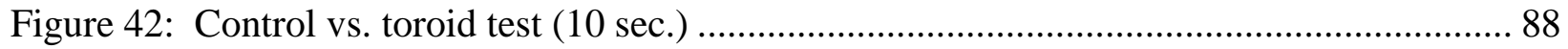

Figure 43: Control vs. toroid test $(20 \mathrm{sec}$.$) .......................................................................... 88$

Figure 44: Control vs. toroid test $(30 \mathrm{sec}$.) ........................................................................ 88

Figure 45: Control vs. tangential north test $(10 \mathrm{sec}$.$) ........................................................... 89$

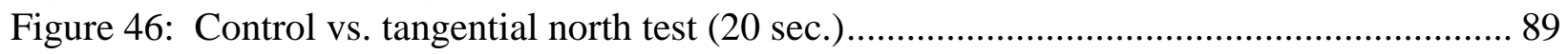

Figure 47: Control vs. tangential north test $(30 \mathrm{sec}$.) .......................................................... 89

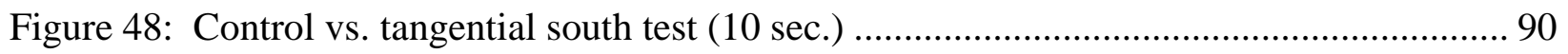

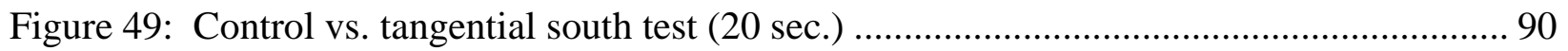

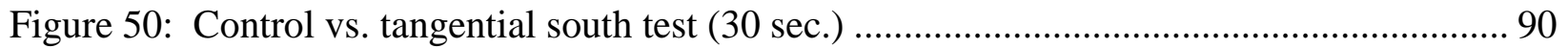

Figure 51: Control vs. axial test $(10 \mathrm{sec}$.$) ....................................................................... 91$

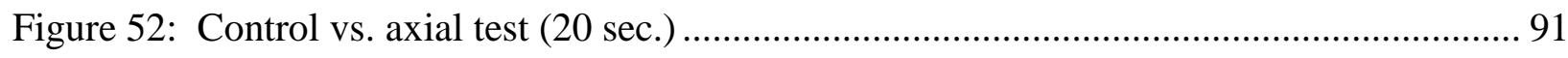

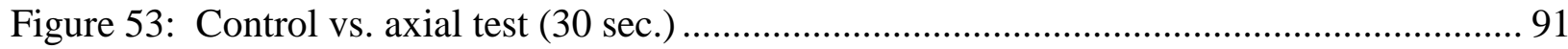

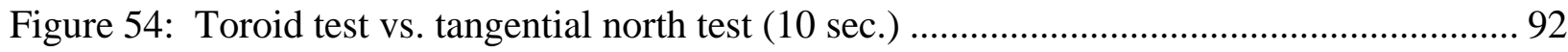

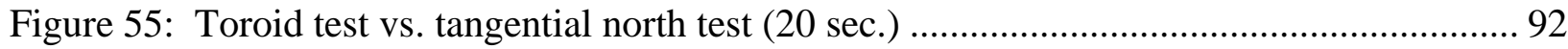

Figure 56: Toroid test vs. tangential north test $(30 \mathrm{sec}$.$) ....................................................... 92$

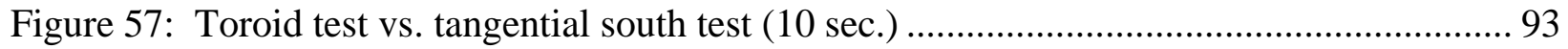

Figure 58: Toroid test vs. tangential south test $(20 \mathrm{sec}$.$) ....................................................... 93$

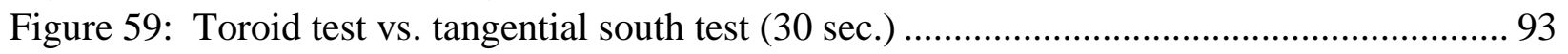

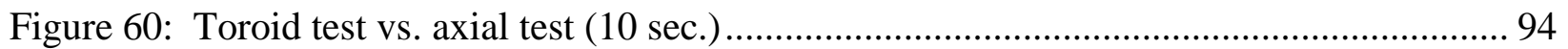

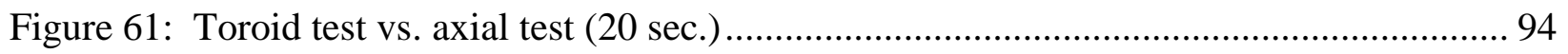

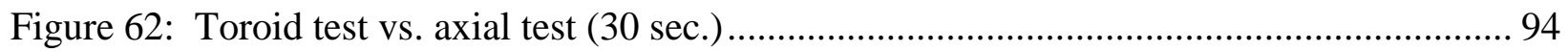




\section{List of Tables}

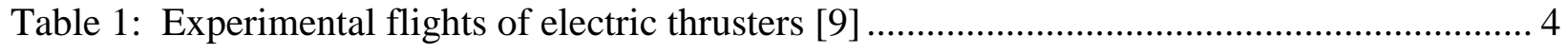

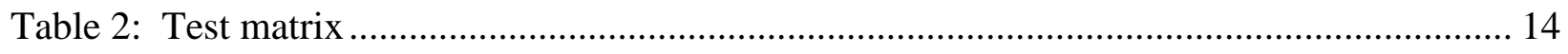

Table 3: Material properties for heat transfer analysis .................................................... 20

Table 4: Experimental temperature values for infinitely, model and plume locations ............... 26

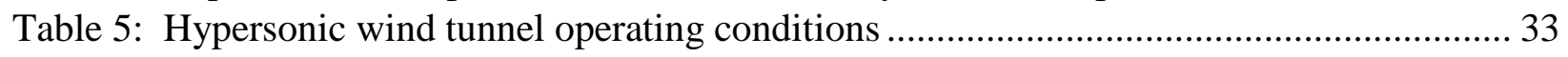

Table 6: Exhaust plume percent decrease from the control test ........................................... 45

Table 7: Exhaust plume percent expansion trends in comparison to the toroid test................. 47 


\section{Nomenclature}

\section{Definitions}

Axial north test

Axial south test

Control test

Tangential north test

Tangential south test

Tangential test

Toroid test
The set of data collected using magnets arranged parallel to the toroid's central axis with the magnets' north poles facing the exhaust nozzle

The set of data collected using magnets arranged parallel to thetoroid's central axis with the magnets' south poles facing the exhaust nozzle

Control data where the arc jet was run without interference from the magnets or the toroidal structure

The set of data collected using magnets arranged orthogonally to the flow direction with the north poles oriented in a clockwise direction The set of data collected using magnets arranged orthogonally to the flow direction with the south poles oriented in a clockwise direction The set of data collected using magnets arranged orthogonally to the flow direction with either the north or south poles oriented in a clockwise direction

The set of data collected using the toroid but no magnets
Abbreviations
CAD
MHD
MPD
WVU
Computer-Aided Design
Magnetohydrodynamic
Magnetoplasmadynamic
West Virginia University

\section{Symbols}

$\begin{array}{lll}\mathrm{A} & = & \text { Cross-sectional area } \\ \mathrm{a} & = & \text { Acceleration } \\ \mathrm{B} & = & \text { Magnetic field strength } \\ \mathrm{Br} & = & \text { Residual flux density } \\ \mathrm{d} & = & \text { Distance } \\ \mathrm{E} & = & \text { Electric field strength } \\ \mathrm{E} & = & \text { Radiation energy emitted } \\ \mathrm{F} & = & \text { Force } \\ \mathrm{I} & = & \text { Electric current } \\ J & = & \text { Current density } \\ \mathrm{J} & = & \text { Joule }\end{array}$




$$
\begin{aligned}
\mathrm{K} & =\text { Temperature in Kelvin } \\
\mathrm{k} & =\text { Thermal conductivity of material } \\
\mathrm{kg} & =\text { Kilogram } \\
\mathrm{m} & =\text { Mass } \\
\mathrm{m} & =\text { Meter } \\
\mathrm{N} & =\text { Number of turns } \\
\dot{Q}_{\text {cond }} & =\text { Conduction heat transfer rate } \\
\mathrm{q} & =\text { Electric charge } \\
\mathrm{R} & =\text { Radius } \\
\mathrm{T} & =\text { Temperature } \\
\mathrm{t} & =\text { Time } \\
\mathrm{V} & =\text { Volume } \\
\mathrm{W} & =\text { Watt }
\end{aligned}
$$

\section{Greek Symbols}

$$
\begin{array}{rll}
\Delta & = & \text { Change in variable } \\
\epsilon & = & \text { Emissivity } \\
\varepsilon & = & \text { permittivity } \\
\mu & = & \text { Permeability } \\
\pi & = & \text { Ratio of a circle's circumference to its diameter } \\
\sigma & = & \text { Stefan-Boltzmann constant }
\end{array}
$$




\section{Introduction}

This thesis investigates the possibility that a toroidal magnetic field can control the exhaust flow emitting from a plasma jet moving at hypersonic speeds as seen in Figure 1. The ultimate objective is to determine if applying the toroidal magnetic field outside the exhaust nozzle of an arc jet thruster can act as a magnetic nozzle by manipulating the plasma flow profile through a vacuum. One potential application for this would be to reduce overexpansion of the exhaust plume in a vacuum. Reducing overexpansion would increase the rocket's net thrust in a single direction. Another potential application would be to purposefully expand the exhaust flow. Under the laws of compressible aerodynamics expanding a compressible flow to a certain degree could potentially increase the total velocity of the flow [1] [2]. A third potential application would be to affect thrust vectoring through controlling the direction of the flow after it exits the exhaust nozzle. Taking advantage of this capability may have potential applications for altering the spacecraft's attitude while concurrently propagating forward motion.

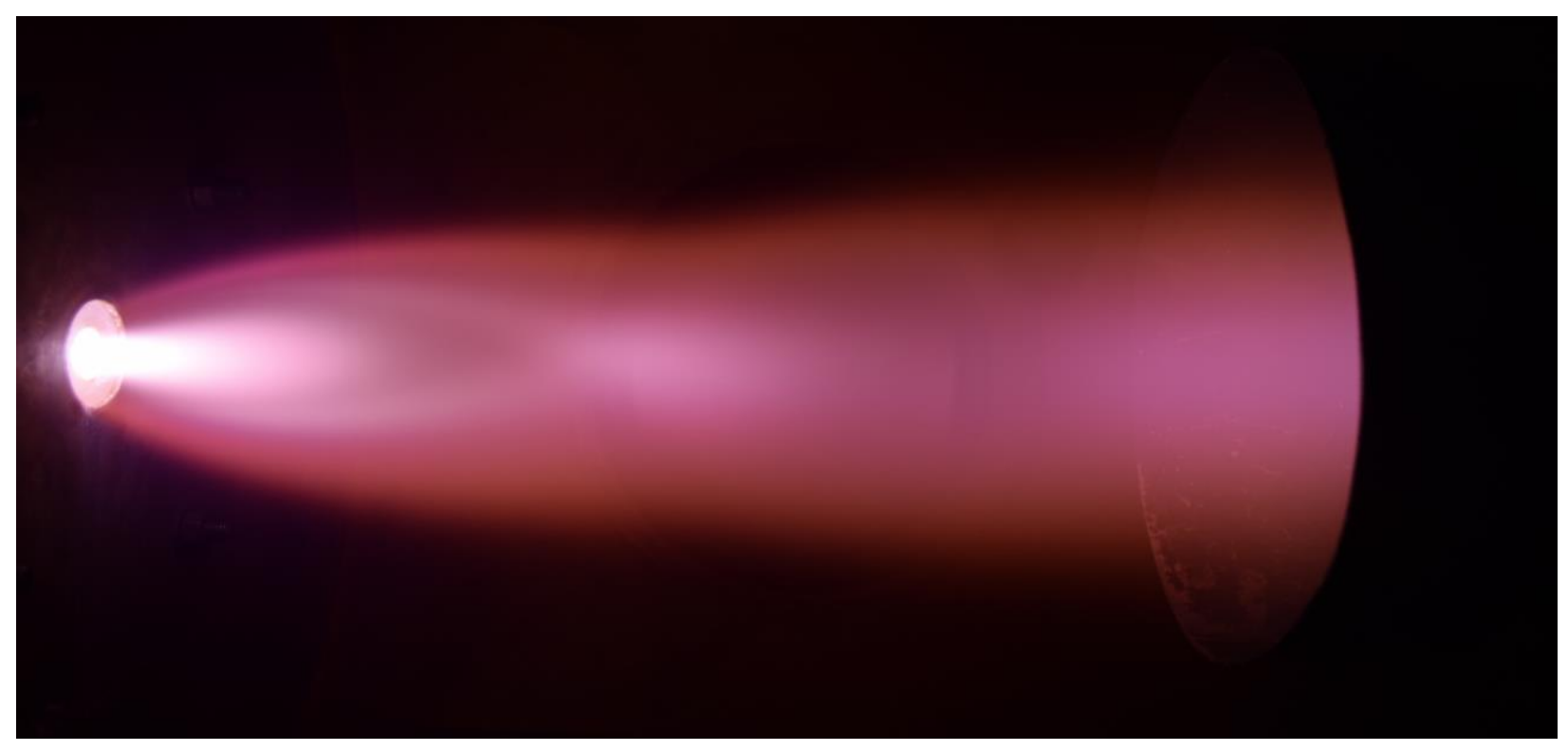

Figure 1: Argon plasma jet

The project utilized a series of twelve N52 grade neodymium magnets placed in different orientations around a steel toroid. This was done to determine which magnetic field configuration tested would have an optimal effect on the flow in different applications and to determine if permanent magnets could serve as a practical replacement for toroidal electromagnets. 
Several tasks had to be accomplished before attempting this experiment. First, the WVU Hypersonic Arc Jet wind tunnel needed to be serviced, and ultimately repaired, before it could run properly. Having a fully functional wind tunnel was crucial because the experiment would not have been possible without it. A literature review was also conducted and several numerical analyses were completed to demonstrate project feasibility. The test apparatus was designed and constructed both physically and digitally using a 3D modeling software prior to completing these analyses. Once the test apparatus was completed, the equipment and procedures necessary for accurate data collection were identified. The experiment metric was also finalized based on the wind tunnel's most effective operating conditions for this project. From there all the necessary equipment was gathered to perform the experiment. Tests of identical operating condition were performed on different test dates and analyses of the experimental results were performed to evaluate the system's sensitivity to repeatability. Results were analyzed using image processing software to determine the best magnetic field configuration for a given application based on the quasi-two-dimensional change in plume contour. 


\section{Problem Statement}

When rockets are launched within the Earth's atmosphere gravity and atmospheric pressure induce forces on the expelled gas. However, in the vacuum of space, gravitational and atmospheric pressure forces become negligible. Without ambient back pressure the nozzle flow is considered under-expanded. That is, the nozzle exit area is insufficient to establish equilibrium between exit static pressure and ambient pressure [3]. When this happens, the compressed gases exiting the nozzle expands both axially and radially. Such expansion produces thrust in a direction contrary to the rocket's trajectory, decreasing its effective thrust capacity and increasing the amount of propellant required for a given mission [3]. This thesis investigates the possibility of using magnetic fields to reduce overexpansion for applications involving electric propulsion systems.

Electric and magnetic fields are a fundamental part of electric propulsion systems [4]. "Electric propulsion can produce thrust by electrically heating propellant, electrostatically accelerating charged particles, or manipulating the flow of charged particles with electromagnetic fields" [5]. The proposed experiment measures the MPD expansion effects of a toroidal magnetic field on an ionized hypersonic exhaust flow.

A secondary project goal is to determine if a series of N52 grade neodymium magnets mounted on a steel toroid can serve as a practical replacement for toroidal electromagnets. "The grade, or ' $\mathrm{N}$ rating' of a magnet refers to the Maximum Energy Product of the material that the magnet is made from" [6]. An N52 grade magnet has a Maximum Energy Product of 52 million Gauss Oersted. These are among the strongest rare earth magnets in existence [6]. While electromagnets can be much larger and stronger than permanent magnets they are often heavier, more expensive, and, most importantly, require external power sources [7]. As such, using strong permanent magnets could be more cost-effective for aerospace structures than electromagnets if they can produce a significant change in the exhaust profile.

Two separate numerical analysis techniques were performed to determine the effectiveness and practicality of this design. First, a MATLAB script was written to predict what effects a given magnetic field would have on the ions and electrons within the argon plasma exhaust plume. A heat transfer analysis was then performed using ANSYS Mechanical to determine the radiation heat transfer effects of the argon exhaust plume on the toroidal structure. This analysis was performed to ensure the neodymium magnets would not exceed their maximum operating temperature. 


\section{Background and Literature Survey}

\section{History of Electric Propulsion}

The first recorded reference of electric propulsion systems for spacecraft was in 1906 when Robert Goddard suggested the possibility of electric propulsion in his personal notebook. In his notebook, Goddard questioned, "At enormous potentials can electrons be liberated at the speed of light, and if the potential is still further increased will the reaction increase (to what extent) or will radio-activity be produced" [8]? Five years later, Konstantin Tsiolkovsky published the first reference to an electric propulsion system stating, "It is possible that in time we may use electricity to produce a large velocity for the particles ejected from a rocket device” [8]. In 1915 and 1920 Goddard was granted patents for designs relating to electric propulsion systems. Although neither of these scientists were able to realize their vision of an electric spacecraft propulsion system, their initial writings show how long scientists have been working toward that goal [8]. While Goddard and Tsiolkovsky may have developed the concept of electric spacecraft it wasn't until the 1960's when the first electric propulsion systems were actually used [9]. Table 1 lists spacecraft launched with electric propulsion as their primary means of producing thrust between 1962 and 1999. Today electric propulsion systems are widely used in rocket design for a variety of applications [10].

Table 1: Experimental flights of electric thrusters [9]

\begin{tabular}{|c|c|c|c|c|c|}
\hline Launch & $\begin{array}{l}\text { Experiment/ } \\
\text { platform }\end{array}$ & $\begin{array}{c}\text { Thruster type/ } \\
\text { number per vehicle }\end{array}$ & $\begin{array}{l}\text { Kilowatts per } \\
\text { thruster }\end{array}$ & Primary data & Sponsor/builder \\
\hline \multicolumn{6}{|c|}{ Subsystems tests: } \\
\hline $1962-64$ & Scout ( 3 suborbital) & Cesium ion/1 & 0.8 & Thruster operations & USAF/EOS \\
\hline 1964 & SERT-I (suborbital) & $\mathrm{Hg}$ ion/1, CS ion/1 & 1.6 & Thruster operations & NASA \\
\hline 1965 & SNAPSHOT & Cesium ion/1 & $<0.5 ?$ & Thruster operations & USAF/EOS \\
\hline 1967 & ATS $-2,-3$ & Ammonia resistojet/2 & $<0.01$ & Thruster operations & NASA/AVCO \\
\hline $1968-69$ & ATS $-4,-5$ & Ammonia resistojet, Cs ion $/ 4$ & $<0.03$ & Thruster operations & $\mathrm{NASA} / \mathrm{AVCO}+\mathrm{EOS}$ \\
\hline 1971 & Sol Rad-10 & Hydrazine resistojet/1 & $<0.01 ?$ & Thruster operations & U.S. Navy/AVCO \\
\hline 1971 & Meteor-10 & $\mathrm{Xe}$ plasma/2; $\mathrm{Hg}$ ion/1 & 0.45 & Thruster operations & Russia/Fakel \\
\hline 1977 & K-9M-58 (suborbital) & Helium MPD arcjet/1 & $0.02 ?$ & Thruster operations & ISAS \\
\hline 1980 & MS-T4 Tansei-4 & Ammonia MPD arcjet/2 & 0.02 & Thruster operations & ISAS \\
\hline 1981 & MDT-2A (suborbital) & Teflon-pulsed plasma/2 & $<0.01$ & Thruster operations & China \\
\hline 1982 & ETS -3 & Mercury ion, $5 \mathrm{~cm} / 2$ & 0.09 & Thruster operations & NASDA/MELCO \\
\hline 1992 & RITA/EURECA-1 & Xenon ion, RIT-10/1 & 0.44 & Thruster operations & ESA/MBB \\
\hline $1998^{a}$ & RHETT-2/EPDM & Xe plasma, TAL D55/1 & 1.5 & Thruster operations & BMDO/TsNIIMash \\
\hline $1999^{a}$ & T-160/Express & Xe plasma, T-160/1 & 4.5 & Thruster operations & $\mathrm{BMDO} / \mathrm{KeRC}$ \\
\hline \multicolumn{6}{|c|}{ Plume and environmental studies: } \\
\hline 1970 & SERT-II & Mercury ion, $15 \mathrm{~cm} / 2$ & 0.84 & Plasma and contamination & NASA \\
\hline 1974 & ATS -6 & Cesium ion $/ 2$ & 0.14 & Charge control & NASA/EOS \\
\hline 1975 & Kosmos-728 & Potassium MPD/1 & 3.00 & Plasma effect & Russia \\
\hline 1981 & ETS -4 & Teflon-pulsed plasma/4 & 0.01 & EMI effect & NASDA/ETL \\
\hline 1983 & SEPAC/Spacelab-1 & Argon MPD/1 & 0.13 & Charge control & ISAS \\
\hline $1980 \mathrm{~s}^{\mathrm{b}}$ & IAPS/P80-1 & Mercury ion, $8 \mathrm{~cm} / 2$ & 0.13 & Plasma and contamination & NASA/Hughes \\
\hline 1987 & Shadow/Kosmos & Cesium $\operatorname{arcjet} / 2$ & 1.5 & Plasma effect & TsNIIMash \\
\hline 1995 & EPEX/SFU-1 & Hydrazine MPD arcjet/1 & 0.43 & EMI and plume & ISAS/IHI + MELCO \\
\hline 1998 & ESEX/ARGOS & Ammonia arcjet/1 & 26.0 & EMI and contamination & USAF/TRW + Primex \\
\hline
\end{tabular}




\section{Types of Electric Propulsion Systems}

There are three primary types of electric propulsion systems used in rockets. These include electro-thermal, electrostatic, and electrodynamic rockets [3]. Electro-thermal rockets generate thrust by converting electrical energy into thermal energy. These rockets use a resister or an electric arc to transfer heat to the rocket's propellant. The gaseous propellant then absorbs the heat and subsequently expands out the exhaust nozzle producing thrust [9]. Examples of electro-thermal rockets include resistojets and arc jet rockets. Resistojet rockets work by running an electric current through a resistor causing it to heat up. The propellant gas then flows over the hot element causing expansion [9]. Arc jet rockets work by passing a propellant over a sustained electric arc producing the same effect as the resistojet rockets [3].

Electrostatic rockets are also known as ion thrusters [3]. These rockets produce thrust by using an electric field to accelerate ionized propellants. However, to avoid generating a negative charge within the thruster the subsequent electrons must also be expelled with the positive ions [3]. This is done by injecting electrons into the exhaust using an anode and an external hollow cathode. Electrons are propelled toward the anode and out the nozzle giving the exhaust a neutral charge [9].

Electrodynamic rockets, also known as plasma thrusters, use a combination of electric and magnetic fields to accelerate the positive ions in an electrically neutral plasma [3]. Two examples of plasma thrusters include hall-effect thrusters and pulsed-plasma thrusters [3]. Hall-effect thrusters utilize an effect called "Hall current" which occurs when a radial magnetic field is applied to a conducting plasma. The applied magnetic field and the electric field within the plasma interact thus causing positive ions within the plasma to accelerate and produce thrust [3]. Unlike most other electric propulsion systems, pulsed-plasma thrusters use a non-continuous electric arc in conjunction with a solid propellant to generate thrust [9]. These thrusters use a capacitor to store energy and periodically release an electric arc. This arc instantly turns the solid propellant into a plasma creating an induced magnetic field. This magnetic field accelerates the plasma through the exhaust nozzle [9]. Diagrams of these, and several other, propulsion systems can be found in Figure 2. 

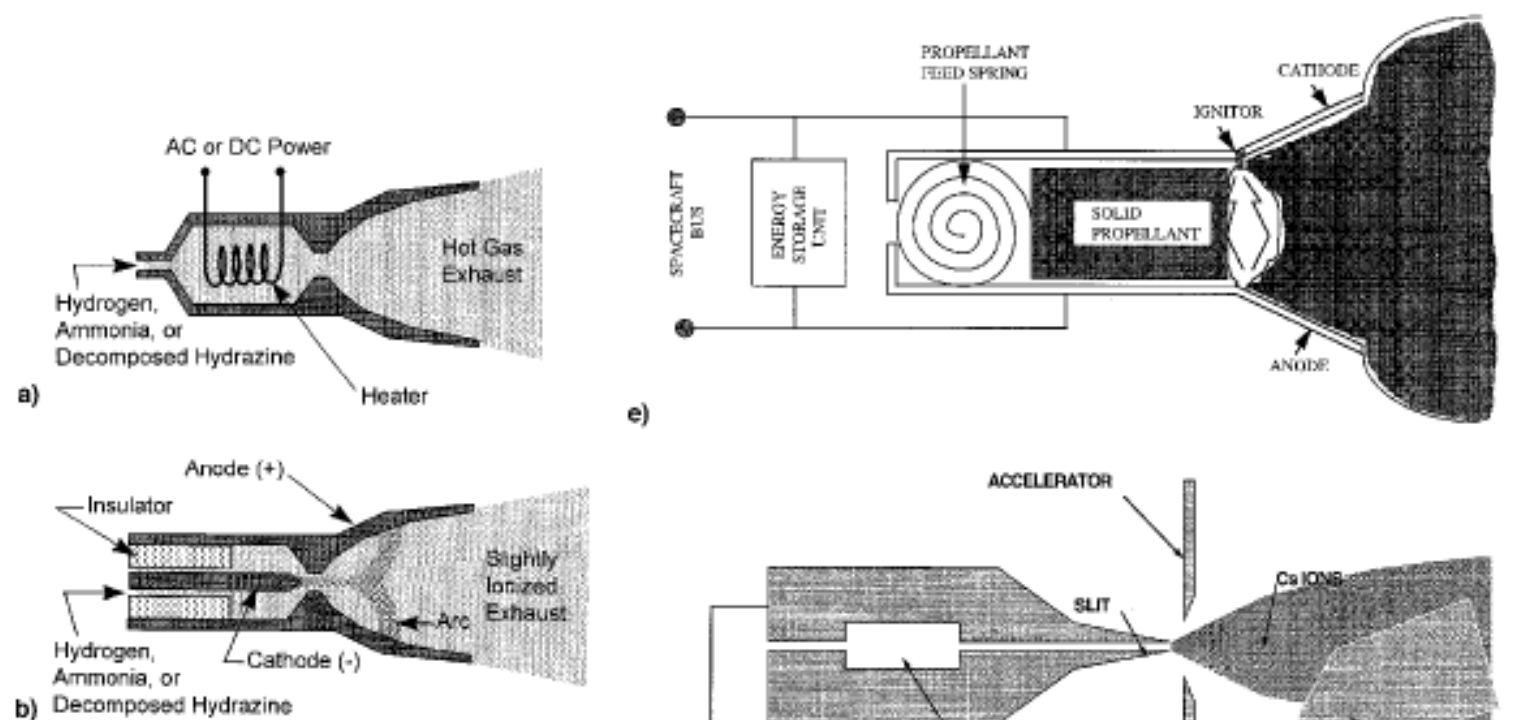

b) Decomposed Hydrazine
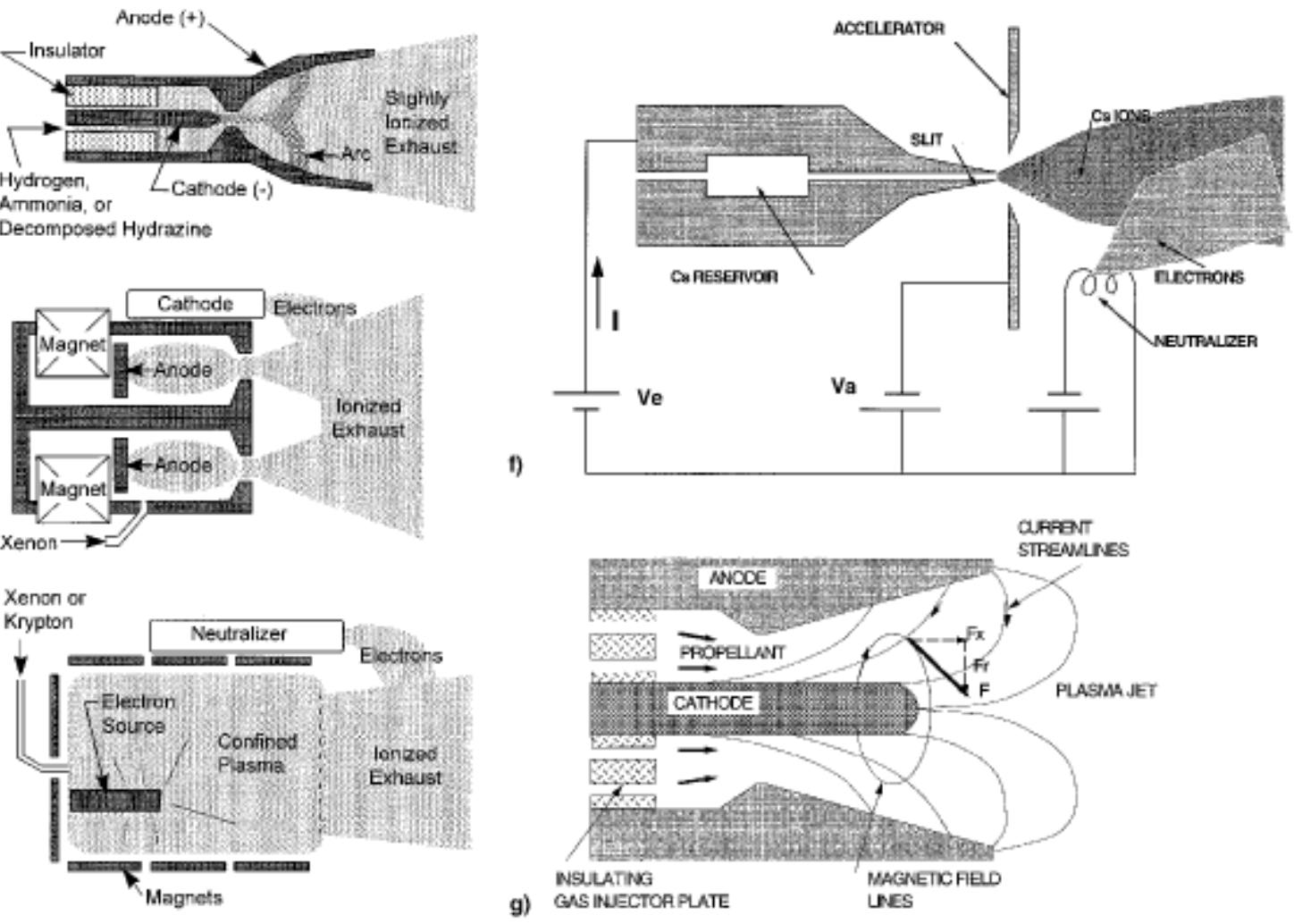

d)

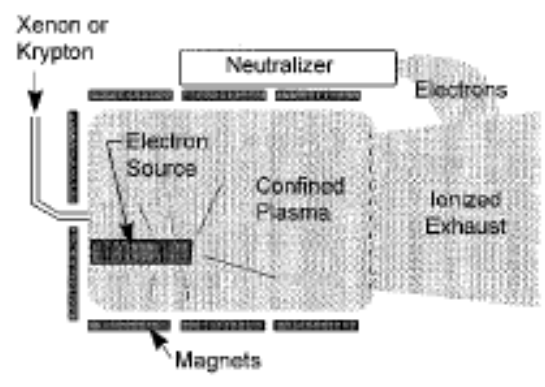

g) GASINUECTORPLATE LNES

Figure 2: “Operating principles of a) resistojets, b) arcjets, c) Hall thrusters, d) ion engines, e) pulsed plasma thrusters, $f$ ) field-effect electrostatic propulsion thrusters, and g) self-field magnetoplasmadynamic thrusters" [9].

\section{Magnetoplasmadynamics and Magnetic Nozzles}

"One method to increase the thrust of an MPD thruster is to operate it in an externally applied magnetic field" [11]. Such applied magnetic fields are known as magnetic nozzles. Magnetic nozzles create thrust by using electric or magnetic fields to direct the flow of ionized particles. This is done by converting thermal and non-directional kinetic energy to directional kinetic energy [5]. The electrostatic force propagated by the field directs a charged propellant through an exhaust nozzle. This is known as electrostatic acceleration. Electrostatic propulsion 
methods incorporate any electric propulsion methods which incorporate magnetic fields. Figure 3 shows the advantages and disadvantages of using electromagnetic propulsion systems compared to electrostatic and other types of rocket propulsion systems.

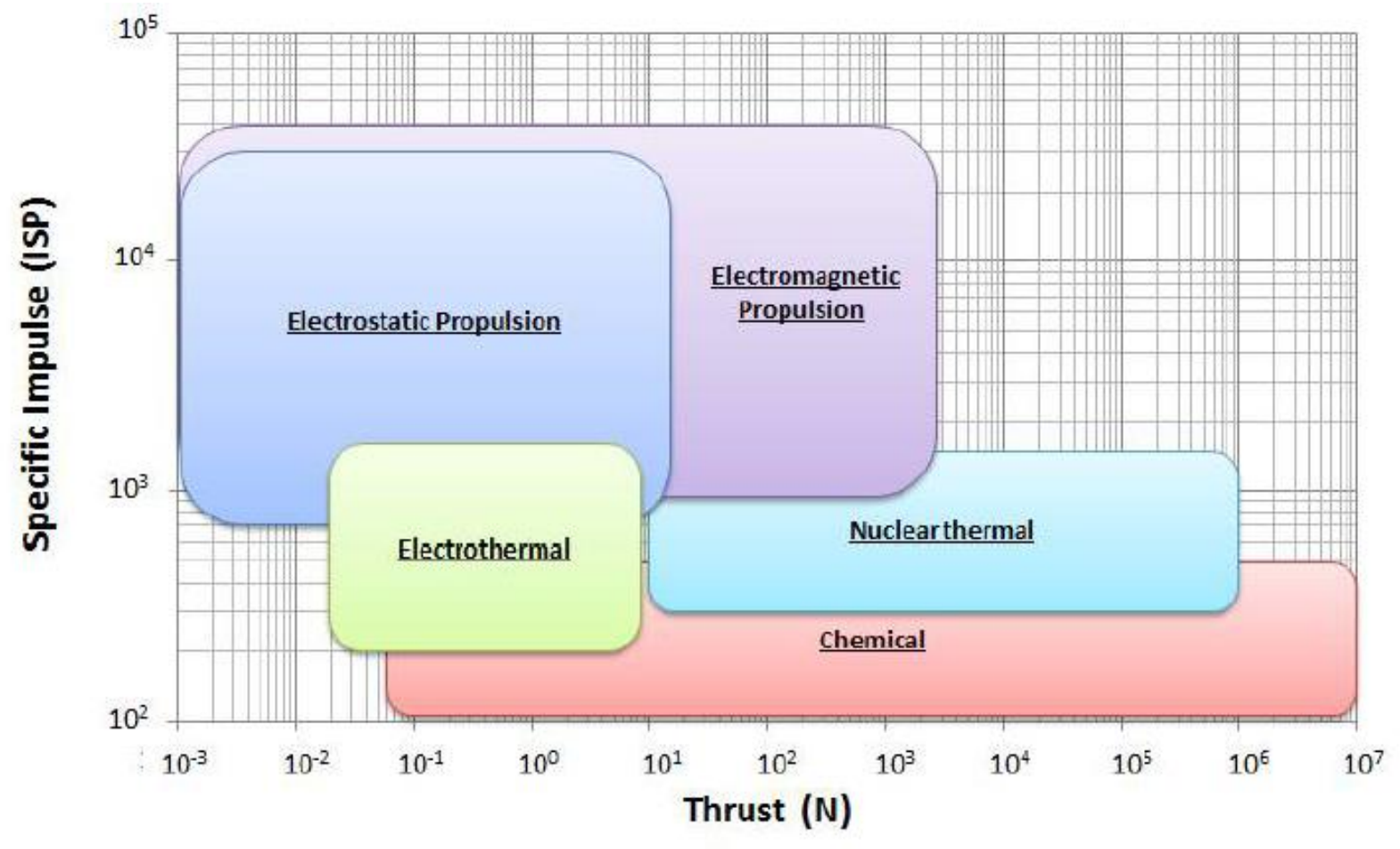

Figure 3: "Specific impulse and thrust comparison for space propulsion methods" [5]

Magnetic nozzles can be applied to various types of electric propulsion systems to produce electromagnetic propulsion. The magnetic fields in these nozzles can be placed in a variety of different locations within the rocket design. The electric or magnetic field can be applied either in the ionization chamber, in the exhaust nozzle, or outside the exhaust nozzle [12]. In this thesis experiment the magnetic field is applied to the flow after it exits the exhaust nozzle for the purpose of reducing overexpansion of the exhaust flow.

There is a set of four equations, called Maxwell's equations, that govern electric and magnetic fields along with the Lorentz force law [13]. Maxwell's equations include Gauss' law for electricity, Gauss' law for magnetism, the Maxwell-Faraday equation, and Ampere's circuit law [14]. Gauss' law for electricity states that the electric flux from a closed surface is equal to the charge enclosed divided by the permittivity of free space [14].

$$
\nabla \cdot \boldsymbol{E}=\frac{\rho}{\varepsilon_{0}}
$$


Gauss' law for magnetism states that the magnetic flux from a closed surface is always equal to zero [14].

$$
\nabla \cdot \boldsymbol{B}=0
$$

The Maxwell-Faraday equation, also called Faraday's law of induction, states that "the line integral of the electric field around a closed loop is equal to the negative of the rate of change of the magnetic flux through the area enclosed by the loop" [14].

$$
\nabla \times \boldsymbol{E}=-\frac{\partial \boldsymbol{B}}{\partial t}
$$

"Ampere's Law states that for any closed loop path, the sum of the length elements times the magnetic field in the direction of the length element is equal to the permeability times the electric current enclosed in the loop" [14].

$$
\nabla \times \boldsymbol{B}=\mu_{0} \boldsymbol{J}+\mu_{0} \varepsilon_{0} \frac{\partial \boldsymbol{E}}{\partial t}
$$

The Lorentz force describes the force applied to a moving charged particle by a magnetic field [14].

$$
\boldsymbol{F}=q \boldsymbol{E}+q \boldsymbol{U} \times \boldsymbol{B}
$$

Together, these formulas are used to describe how magnetic nozzles effect the ionized particles moving through all electric propulsion systems [13].

\section{WVU Hypersonic Arc Jet Wind Tunnel}

A significant amount of time was spent understanding the wind tunnel operating procedures and gathering all the historic documents and other operational notes related to the wind tunnel. The hypersonic arc jet wind tunnel at WVU was designed and built by Dr. John Loth during the late 1960s [15]. In its most basic description the tunnel comprises an arc jet assembly (Figure 4), a vacuum chamber (Figure 5), a control console (Figure 24), a dual stage vacuum pump and roots blower system, and a model SR-1500-F7 Miller DC power supply [15]. 


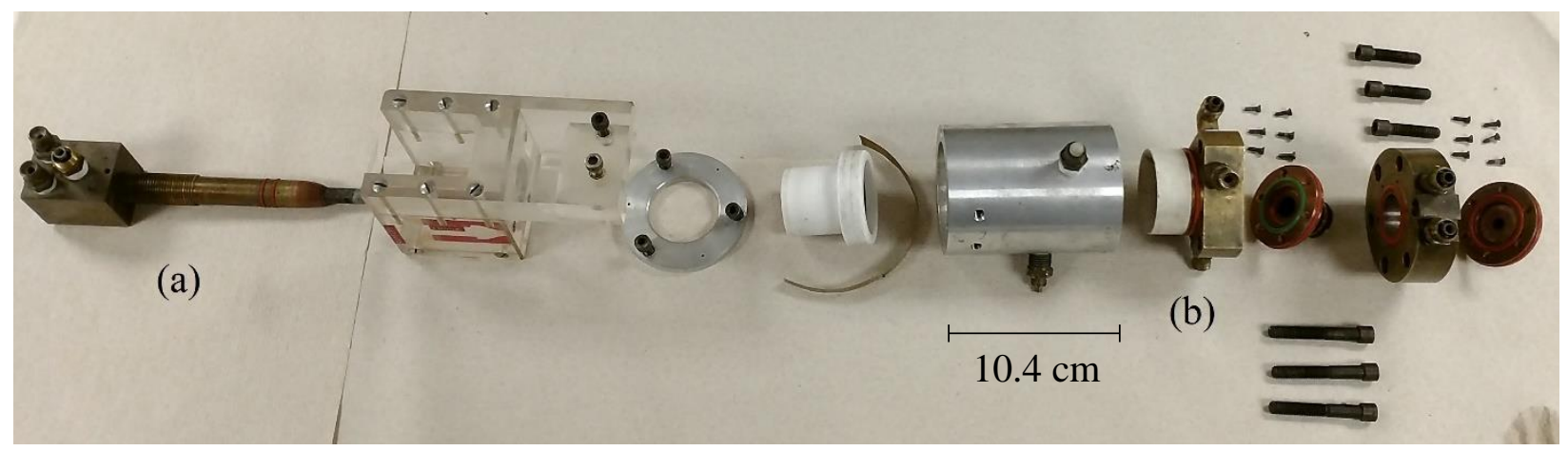

Figure 4: Dismantled arc jet assembly with (a) cathode and (b) anode

The necessary operating procedure was pieced together primarily from a 1973 thesis transcript by Leonard E. Graham entitled, "Feasibility Study of Enthalpy, Density, and Velocity Probe for Low Density Supersonic Flows" [15]. This document was treated as a user manual because it contains a detailed description of the tunnel and its operating procedures.

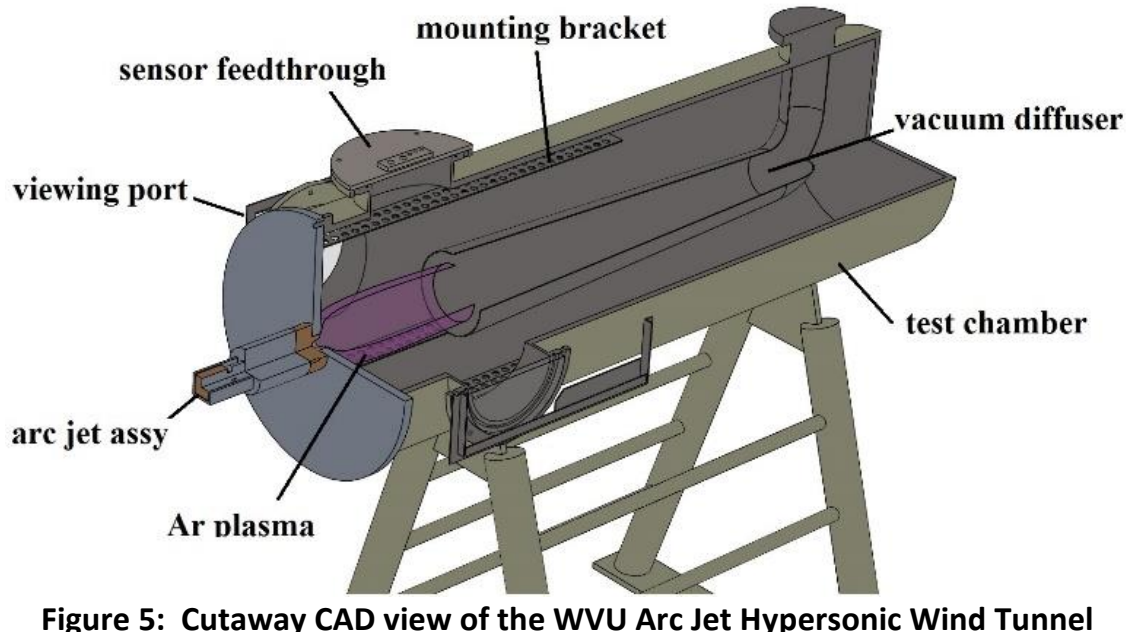

An arc jet rocket is a type of MPD thruster that converts electrical energy into thermal energy by passing a propellant gas through an electric arc [4]. Arc jet rockets operate as low thrust propulsion systems that have a high specific impulse [11]. The arc jet depicted in Figure 4 acts as a "point-plane" wind actuator to propel the ionized gas into the vacuum chamber [16]. In this actuator, the point is the tungsten tipped cathode (Figure $4 \mathrm{a}$ ) and the plane is the anode converging nozzle (Figure 4b) [15]. A corona discharge forms at the point of the cathode and reaches toward the anode producing an electric arc. This arc heats and ionizes the surrounding gas producing plasma. The same electric field also serves to accelerate the plasma toward the anode until it exists the exhaust nozzle [16]. "The plasma flow is assumed to be governed by the Navier-Stokes equations coupled with the magnetohydrodynamics (MHD) equations for [radio frequency] 
electromagnetic field" [17]. "Plasma flow velocity and Mach number increase downstream as the ion temperature decreases" [5].

Once the plasma exits the exhaust nozzle it enters the vacuum chamber for a distance of approximately $41 \mathrm{~cm}$ before entering the diffusion nozzle. The water-cooled diffusion nozzle connects the duel stage vacuum and roots blower system and is responsible for evacuating the vacuum chamber [15]. The vacuum chamber pressure is calculated by subtracting the gage pressure on the console from the ambient pressure.

\section{Methodology and Apparatus Design}

The purpose of this project is to manipulate the flow profile of an ionized exhaust plume under near vacuum conditions using a toroidal magnetic field. Specifically, the study investigates the argon plasma jet produced by the WVU Hypersonic Arc Jet Facility. During this experiment the argon plasma was directed through a steel toroid before entering a diffusion nozzle connected to the vacuum pump. The steel toroid was located approximately $24.2 \mathrm{~cm}$ from the exhaust nozzle and $14.5 \mathrm{~cm}$ from the diffuser nozzle for all tests.

The test apparatus was designed and constructed both physically and digitally using the SolidWorks 3D modeling software. Developing a 3D computer-aided design (CAD) model of the testing apparatus prior to its physical construction helped determine the best way to mount the toroid within the wind tunnel and the size of each part. A CAD model of the apparatus bolted to mounting brackets found within the WVU Hypersonic Arc Jet Wind Tunnel is displayed in Figure 6. 


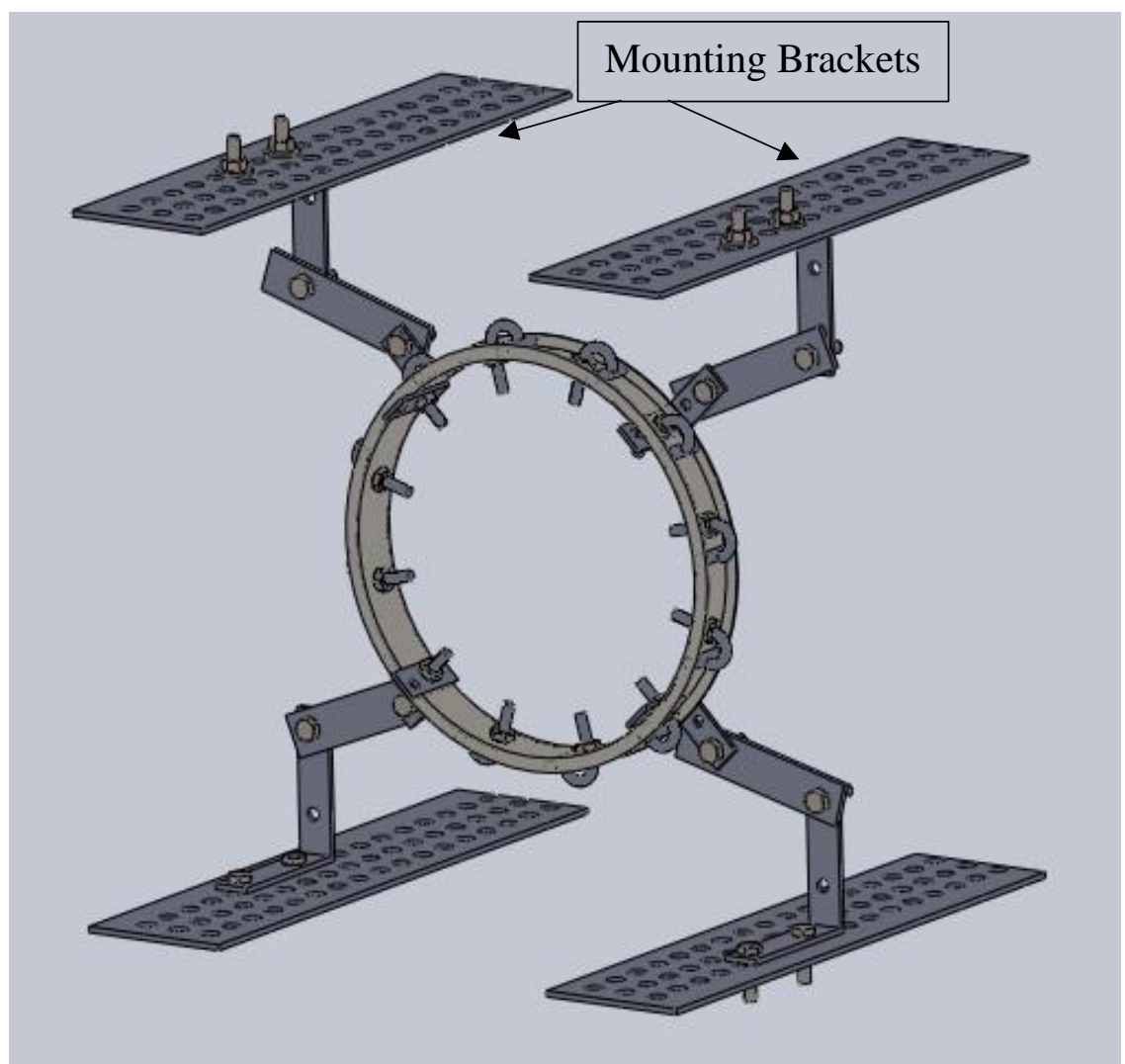

Figure 6: Full scale CAD model of the test apparatus

Adhering to the design proposal as a low-cost alternative to toroidal electromagnets, the testing apparatus assembly was constructed primarily from low-cost materials found at ACE Hardware. The steel toroid was modified from a shopping cart wheel matching previously determined design specifications. The wheel had an inner diameter of approximately 8.5" (21.59 $\mathrm{cm})$ and was supported with 12 steel spokes as seen in Figure 7. 


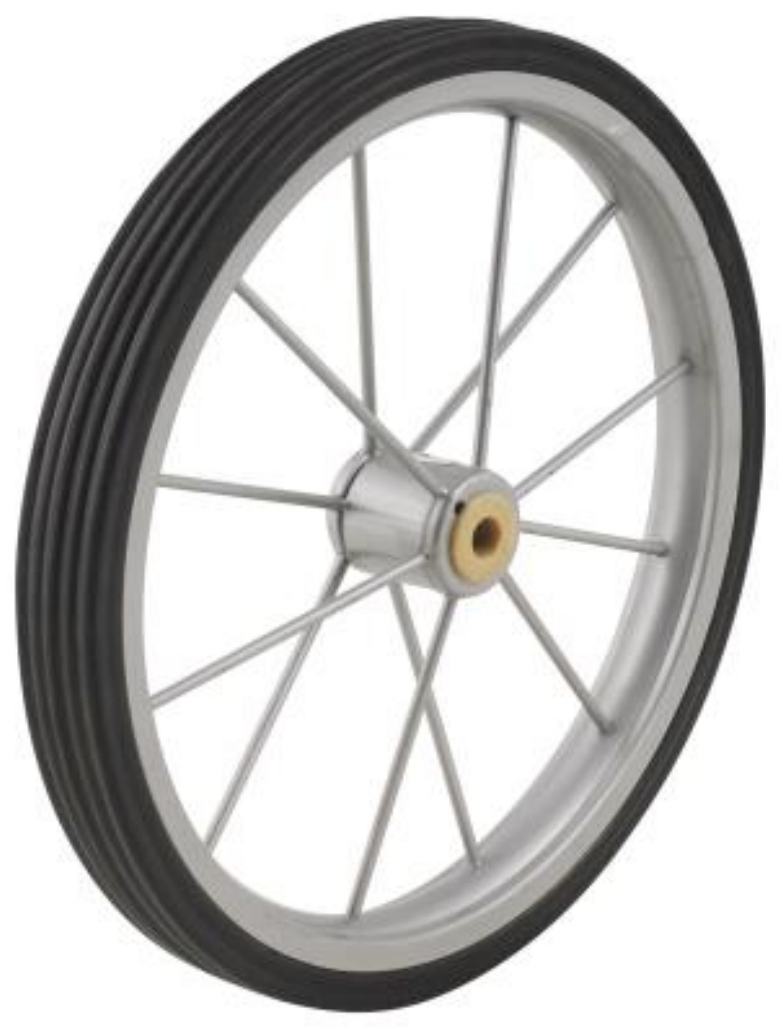

Figure 7: Unmodified shopping cart wheel from ACE Hardware [18]

The tire and spokes were removed and the resulting holes were repurposed to fit $12 \mathrm{~J}$ bolts which were used as magnet fasteners. These bolts were also used to mount the toroidal structure as seen in Figure 6 and Figure 8. 


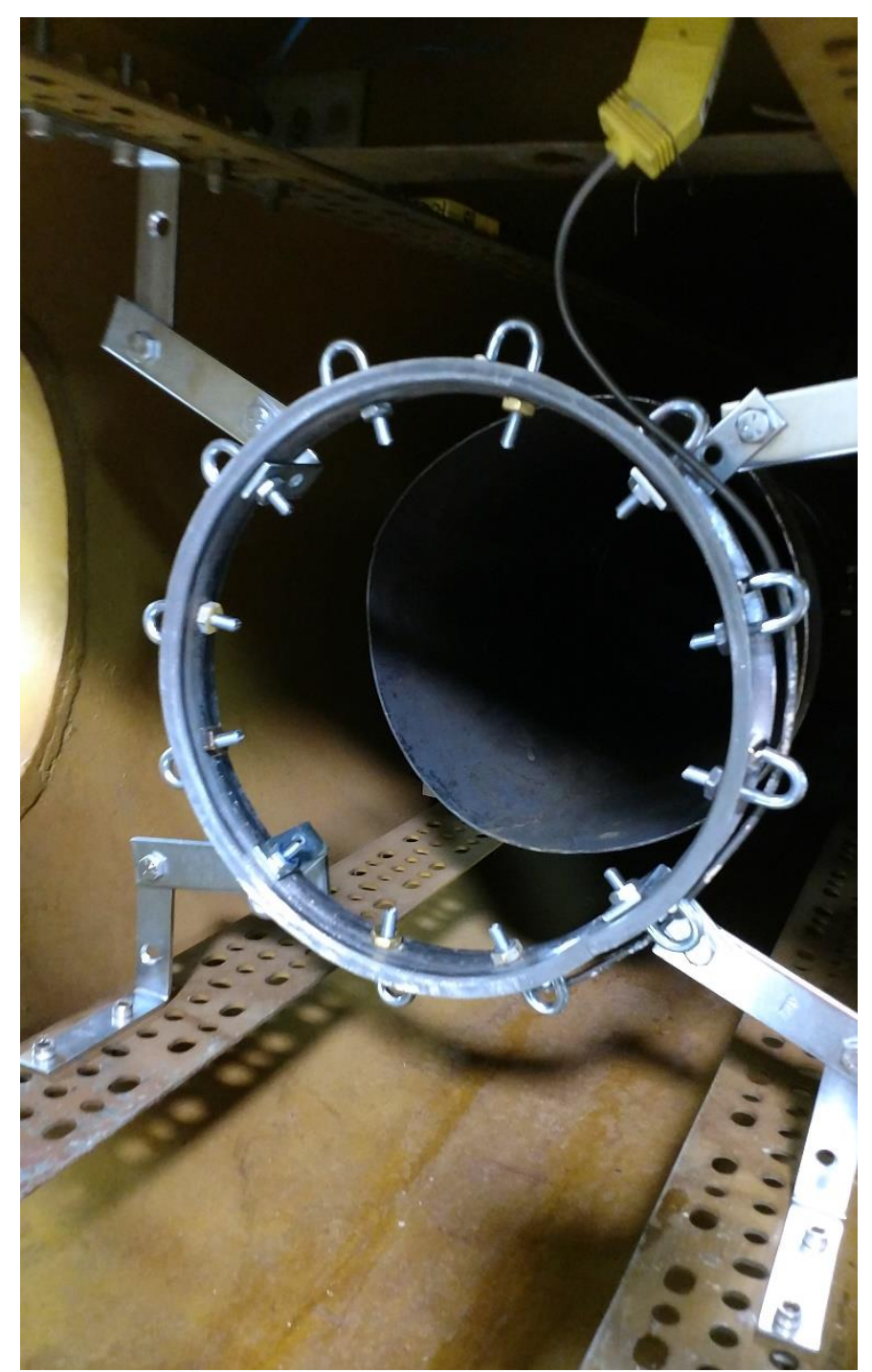

Figure 8: Toroid mounted in wind tunnel

Steel brackets were fastened to the toroid using standard fastener hardware and the model's brackets were subsequently mounted to four permanent brackets fixed inside the tunnel's test section.

Apart from the magnets, argon gas, wind tunnel, and the laptop used for data collection and analysis all other equipment was borrowed from the project advisor. Such equipment included a temperature gage, three Type-K thermocouples, electrical wiring, a Nikon D5300 camera, a tripod, nuts, bolts, and washers. The camera was connected to the laptop to remotely capture images without altering its position while the thermocouples monitored the toroid temperature within the vacuum chamber to ensure the magnets did not reach their maximum operating temperature. 
The test apparatus utilized a series of twelve N52 grade neodymium magnets mounted on the steel toroid. Six different apparatus configurations were tested in this experiment. Two configurations were run as control tests without any magnetic interference. The control tests were run with and without the steel toroid to determine if deploying a toroidal structure alone would influence the exhaust flow independently of a magnetic field. Two data sets were collected with the north and south magnetic poles, respectively, facing the exhaust nozzle. For each of these tests the north poles of all magnets were oriented in the same direction with respect to one another. This was done to determine what effects the magnetic field would have on the exhaust flow if the magnetic field lines were parallel to the direction of the exhaust velocity. The fifth and sixth data sets involved orienting the magnets tangentially with respect to the exhaust flow. For these tests the magnets' north poles uniformly faced either clockwise or counterclockwise around the toroid. This was done to determine what effects the magnetic field would have on the exhaust flow if the magnetic field lines were tangential to the direction of the exhaust velocity. All magnets in each data set were positioned equidistant to one another and equidistant to the toroid's central axis. A test matrix representing the order in which tests were performed is presented in Table 2. Because magnetic field lines run from north to south, the orientation of the poles were switched to determine if the direction of the magnetic field lines affected the exhaust flow for each magnetic field orientation.

Table 2: Test matrix

\begin{tabular}{|c|c|c|c|}
\hline $\begin{array}{c}\text { Control Test } \\
\text { (no magnets) } \\
\text { (no toroid) }\end{array}$ & Nagnet Orientation & Test Numbers & Time Steps (sec.) \\
\hline $\begin{array}{c}\text { Toroid Test } \\
\text { (no magnets) }\end{array}$ & N/A & $19-24,67-72$ & $10,20,30$ \\
\hline Axial Tests & Axial North & $1-6,43-48$ & $10,20,30$ \\
\hline Tangential Tests & Axial South & $13-18,31-36$ & $10,20,30$ \\
\hline & $\begin{array}{c}\text { Tangential North } \\
\text { Tangential South }\end{array}$ & $37-42,61-66$ & $10,20,30$ \\
\hline
\end{tabular}

Using permanent magnets for this experiment was lower cost and more versatile than designing and purchasing electromagnets of similar size and strength. This method of using permanent magnets in different orientations also permitted testing under various magnetic field configurations without constructing an entirely different apparatus. Multiple electro magnets 
would have been required to study the same number magnetic fields shown in Table 2 which would have further increased the project cost.

An equation based on Ampere's law:

$$
\mathrm{N}=\frac{2 \pi r B}{\mu I}
$$

was used to calculate the number times a 18 gauge copper wire would need to turn around an iron core of similar dimensions as the steel toroid used in this experiment. Assuming the steel toroid and iron core had similar weights and dimensions, and assuming both magnets produced the same maximum magnetic field, the total weight of the electromagnet would be approximately 58 grams heavier than the permanent magnets. The weight of the copper wire required for this calculation was approximately 130 grams while the twelve neodymium magnets only had a combined weight of 72 grams. Furthermore, the calculated weight of the electromagnet does not include the weight of a power supply large enough to support the magnet over a large period of time. Depending on the application, adding the extra weight to obtain a higher magnetic field strength may be practical for short term thrust. However, the necessary power source required to support an electromagnet for long term missions would require too much extra mass to satisfy Newton's second law of motion:

$$
a=\frac{F}{m}
$$

compared to using permanent magnets capable of producing the same amount of thrust.

The most effective configuration was determined by comparing data from each orientation in juxtaposition with other data sets. Each test was documented using 12 sets of photographs taken from a fixed position with respect to the flow. Photographs were taken after the arc jet had run for 10,20 , and 30 seconds because the time interval of the plume was of interest. Those specific time periods were chosen because the camera operated most effectively in 10 second intervals and the arc jet could not be run for longer than 30 seconds. Data from each configuration was compiled using image processing and compared with data from other configurations at corresponding time periods. Data from all time periods of each set was compared to determine if the flow was time variant. 


\section{Numerical Analysis}

\section{MATLAB Analysis}

A numerical analysis was performed to verify concept feasibility. Figure 9 reveals a significant difference in the effect magnetic fields have on negatively charged electrons versus the positively charged ions within the argon plasma.

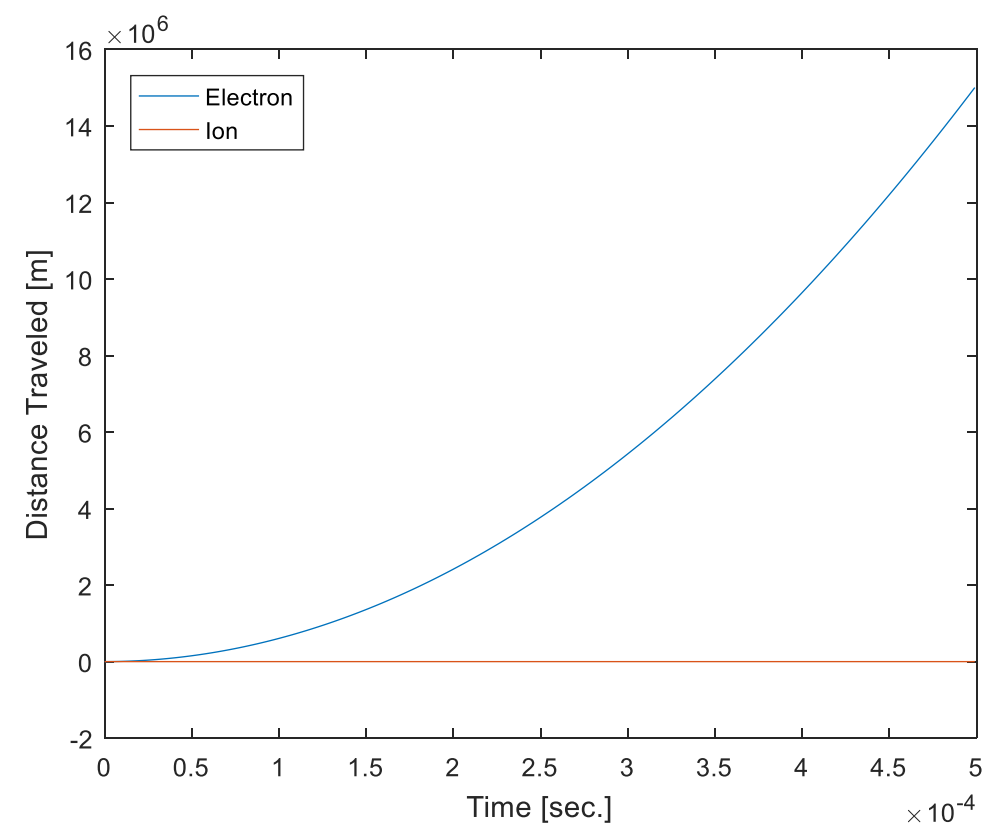

Figure 9: Movement of charged particles based on a 0.7343 Tesla magnetic field

These calculations were based primarily on Equation 7

where the acceleration of a particle is equal to the total force applied to that particle divided by its mass and the Lorentz force law from Equation 5. Electrons are much more susceptible to velocity changes from applied magnetic fields because they are considerably less massive than the positive ions [13]. In this analysis, the effect of the magnetic field on the position of argon ions was found to be negligible.

Further analysis also revealed an exponential decay in magnetic field strength as distance from the magnet increased linearly. This analysis was based on the equation for magnetic field strength:

$$
B=\frac{B r V}{2 \pi d^{3}}
$$


where $d$ is the distance away from the magnet. Results from this analysis are depicted in Figure 10 where the magnet radius is approximately $0.3 \mathrm{~cm}$. The minimum magnetic field at the edge of the plasma plume, about $2 \mathrm{~cm}$ away from the magnet, was approximately 237 Gauss.

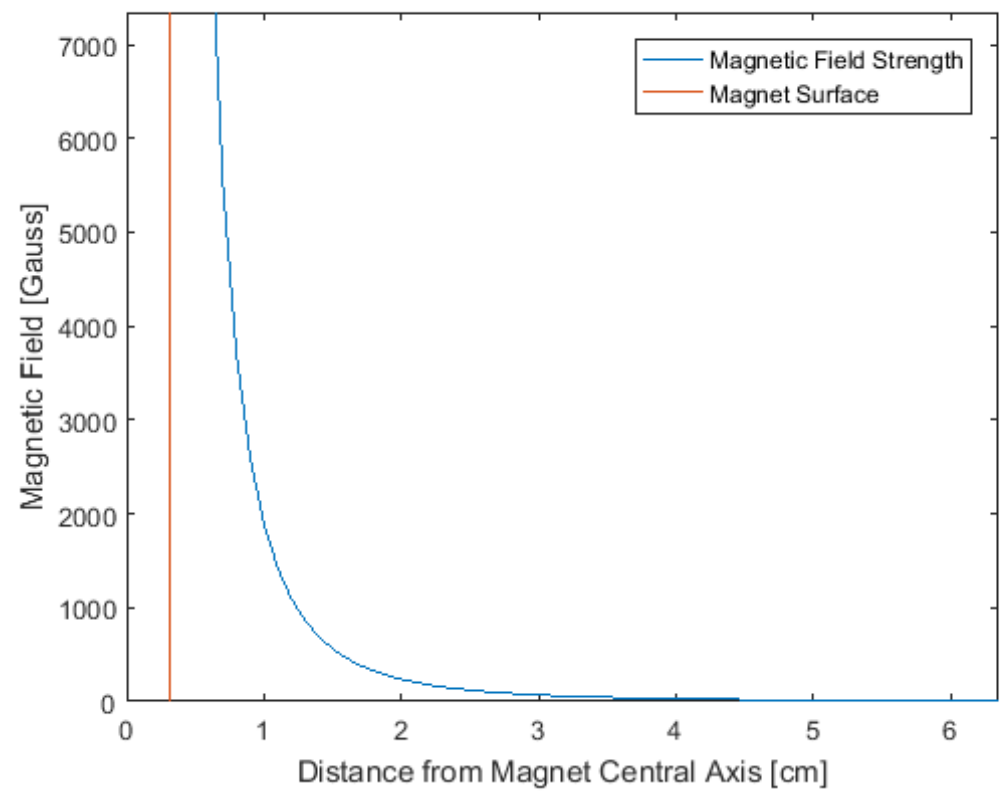

Figure 10: Decay of a magnetic field [Tesla] as distance [m] from the magnet's central axis increases

The code developed for this analysis can be found in Appendix A. Data from this analysis is supported by technical data and figures, such as Figure 11, provided by the K\&J Magnets website, where the magnets were purchased. Figure 11 provides a two-dimensional view of the magnetic field decay, from Figure 10, around the entire magnet. 


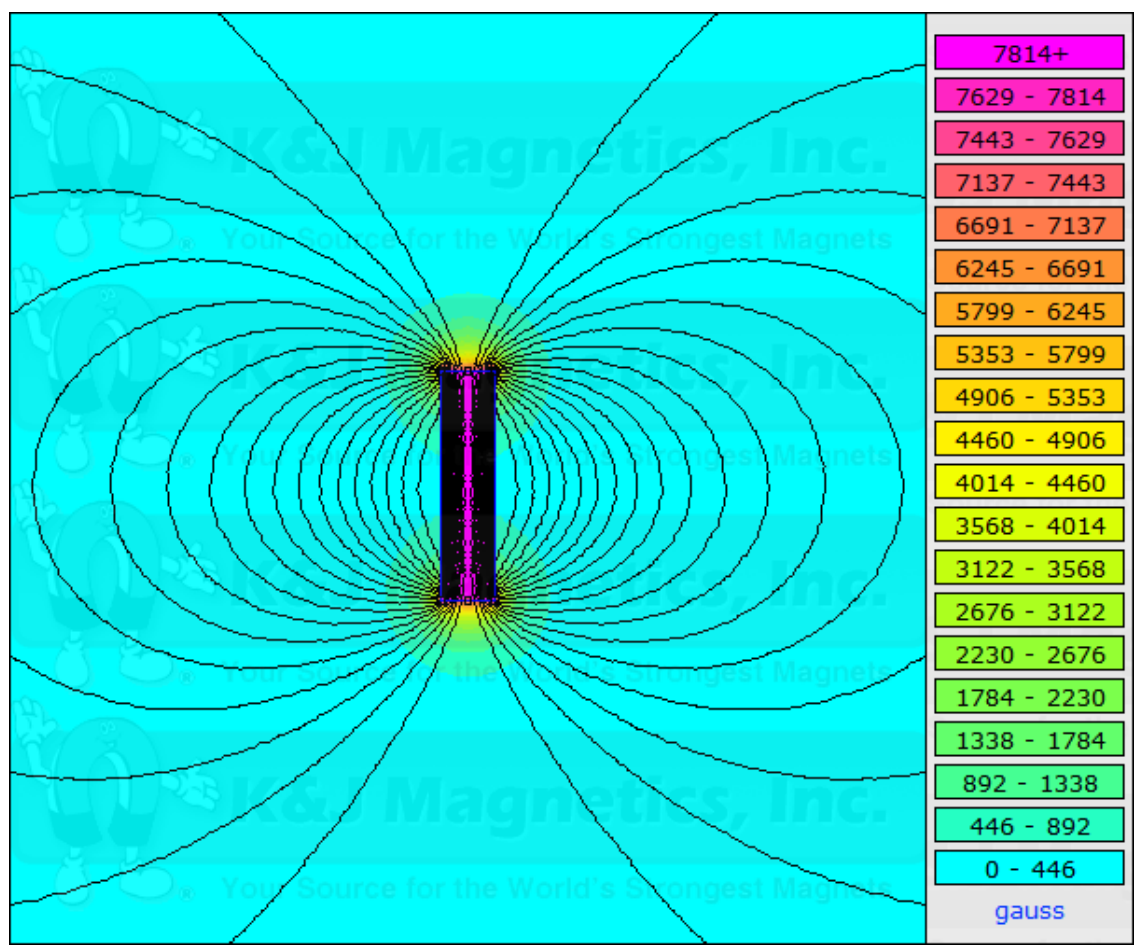

Figure 11: Magnetic field lines for a single magnet in free space [6]

However, this analysis only accounts for a single magnet. The analysis does not account for alterations in magnetic field lines due to interactions between magnets or between a magnet and the steel testing apparatus.

Figure 12 depicts the magnetic field through a toroidal structure. The magnetic field in Figure 12 spans a much greater area than the field in Figure 11. The magnetic field through the toroid is stronger because opposing sides on the interior of the magnet interact with one another. The magnetic field through a toroid spans a much greater area than the magnetic field from a single magnet for the same reason. Since the numerical analysis described in this section only considers the field due to a single magnet, the wider magnetic field through the toroidal test apparatus should have a greater influence on the plasma. 


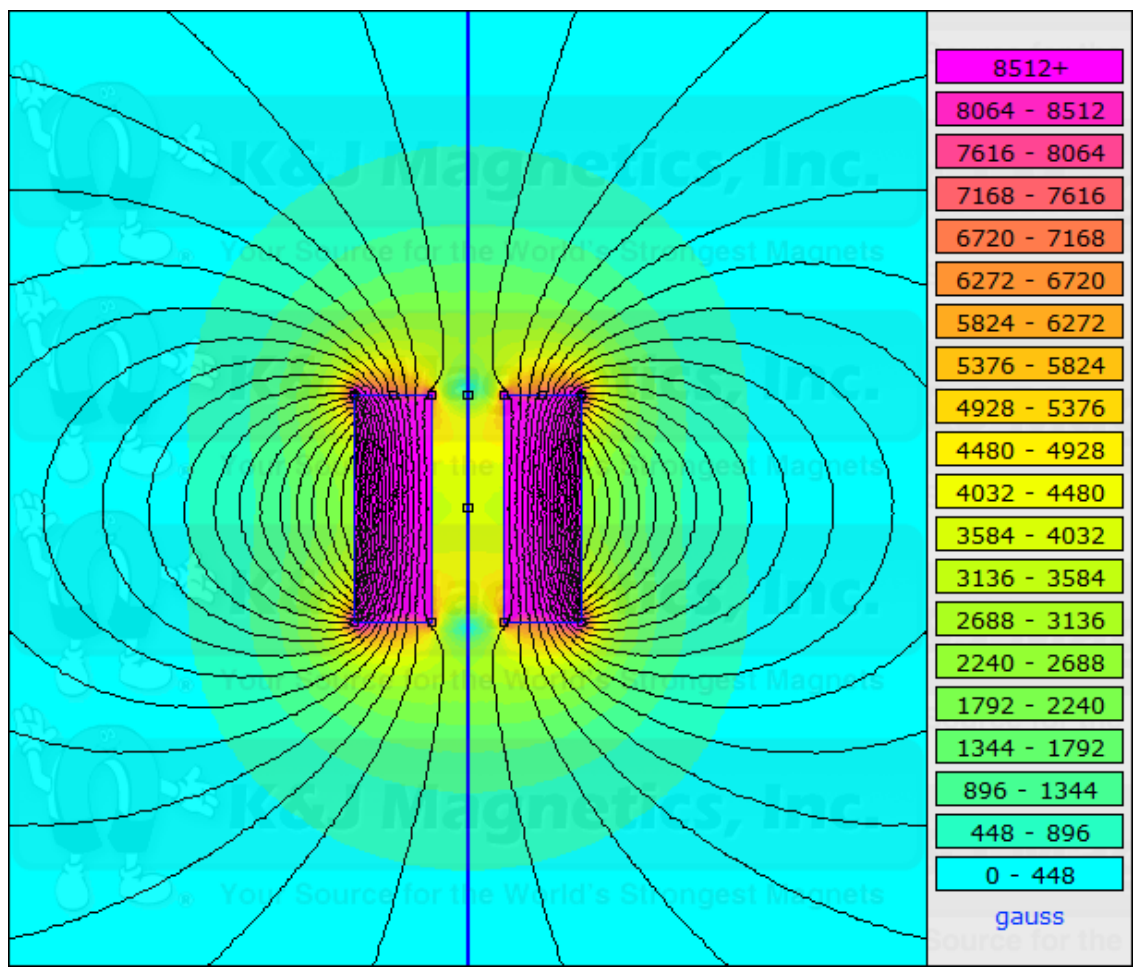

Figure 12: Magnetic field lines through a toroidal structure [6]

\section{Heat Transfer Analysis}

\section{Numerical Setup}

A heat transfer numerical analysis was completed to determine the test apparatus temperature profile due to the ionized exhaust plume using ANSYS Mechanical. However, before any numerical analysis could begin a three-dimensional model of the test apparatus had to be developed. This was done using SolidWorks. The SolidWorks assembly was then imported to ANSYS Workbench as a SAT file as depicted in Figure 13. 


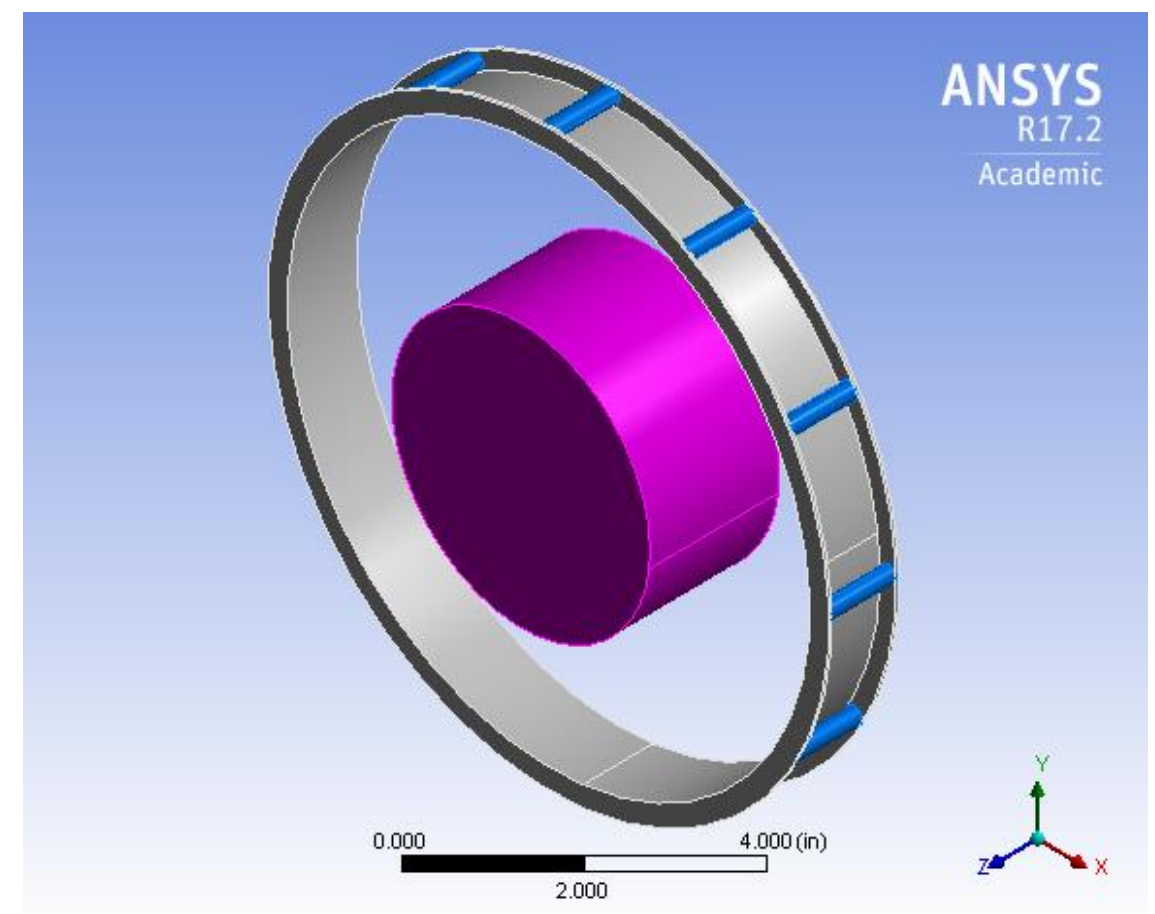

Figure 13: Analysis geometry

The purple section in Figure 13 represents the argon plasma and each blue cylinder represents a neodymium magnet. The material properties of neodymium were manually inserted into ANSYS as a custom material using an online database [19] in conjunction with material specifications listed on the K\&J Magnets website where physical specimens were purchased [6].

Table 3: Material properties for heat transfer analysis

\begin{tabular}{|c|c|c|c|}
\hline & Argon & Neodymium & Structural Steel \\
\hline $\begin{array}{l}\text { Density } \\
{\left[\mathrm{kg} /\left(\mathbf{m}^{3}\right)\right]}\end{array}$ & 1.62 & 7003 & 7850 \\
\hline $\begin{array}{l}\text { Reference Temperature } \\
{[\mathrm{K}]}\end{array}$ & 878 & 290 & 290 \\
\hline $\begin{array}{l}\text { Specific Heat } \\
{[\mathrm{J} /(\mathbf{k g} * \mathbf{K})]}\end{array}$ & 521 & 190 & 434 \\
\hline $\begin{array}{l}\text { Isotropic Thermal Conductivity } \\
{\left[\mathbf{W} /\left(\mathbf{m}^{*} \mathbf{K}\right)\right]}\end{array}$ & 0.02 & 13 & 60.5 \\
\hline
\end{tabular}

The ANSYS simulation was run using a total of 15301 nodes and 4922 elements. The argon plasma plume maintained a constant temperature of $878 \mathrm{~K}$ while the initial temperature of the toroid was set at $306 \mathrm{~K}$. Radiation heat transfer was evaluated between the argon plume and the innermost surface of the steel toroid using emissivities of 0.2 and 0.07 , respectively [20] [21]. To simplify calculations, the entire test apparatus depicted in Figure 6 was not evaluated. Several 
conduction heat transfer sources were purposefully omitted to compensate for any unknown factors which would increase the heat transfer unexpectedly. Limiting the conduction heat transfer served to increase the maximum toroidal temperature, thus increasing the acceptable margin of error between numerical and experimental temperature values. Radiation heat transfer from the toroid's outermost surface to its surroundings was also omitted for the same reason. This evaluation represents a worst-case scenario allowing researchers to also calculate the maximum timespan the apparatus could be exposed to continuous radiation heat transfer before reaching the magnets' maximum operating temperature of $353 \mathrm{~K}$.

\section{Experiment}

Three Type-K thermocouples were used in conjunction with a multichannel thermometer to measure key temperatures during this part of the experiment. One sheathed and grounded 3/32" probe (time constant, $\mathrm{t} \sim 0.23 \mathrm{sec}$ ) was placed in a region of the test section that was not exposed to the argon plasma plume to measure ambient temperature, $T_{i n f}$. A second grounded 3/32" probe was fixed firmly to the outside radius of the toroid, also unexposed to the argon plasma, to measure the toroid model temperature, $T_{\text {mod }}$. A sheathed and ungrounded 1/4" probe $(\mathrm{t} \sim 2.2 \mathrm{sec})$ was placed directly in the stream of the argon plasma to measure the temperature of the plume, $T_{\text {plume }}$. Figure 14 demonstrates how the model was mounted in the tunnel and shows the location of the three thermocouples used during the tests.

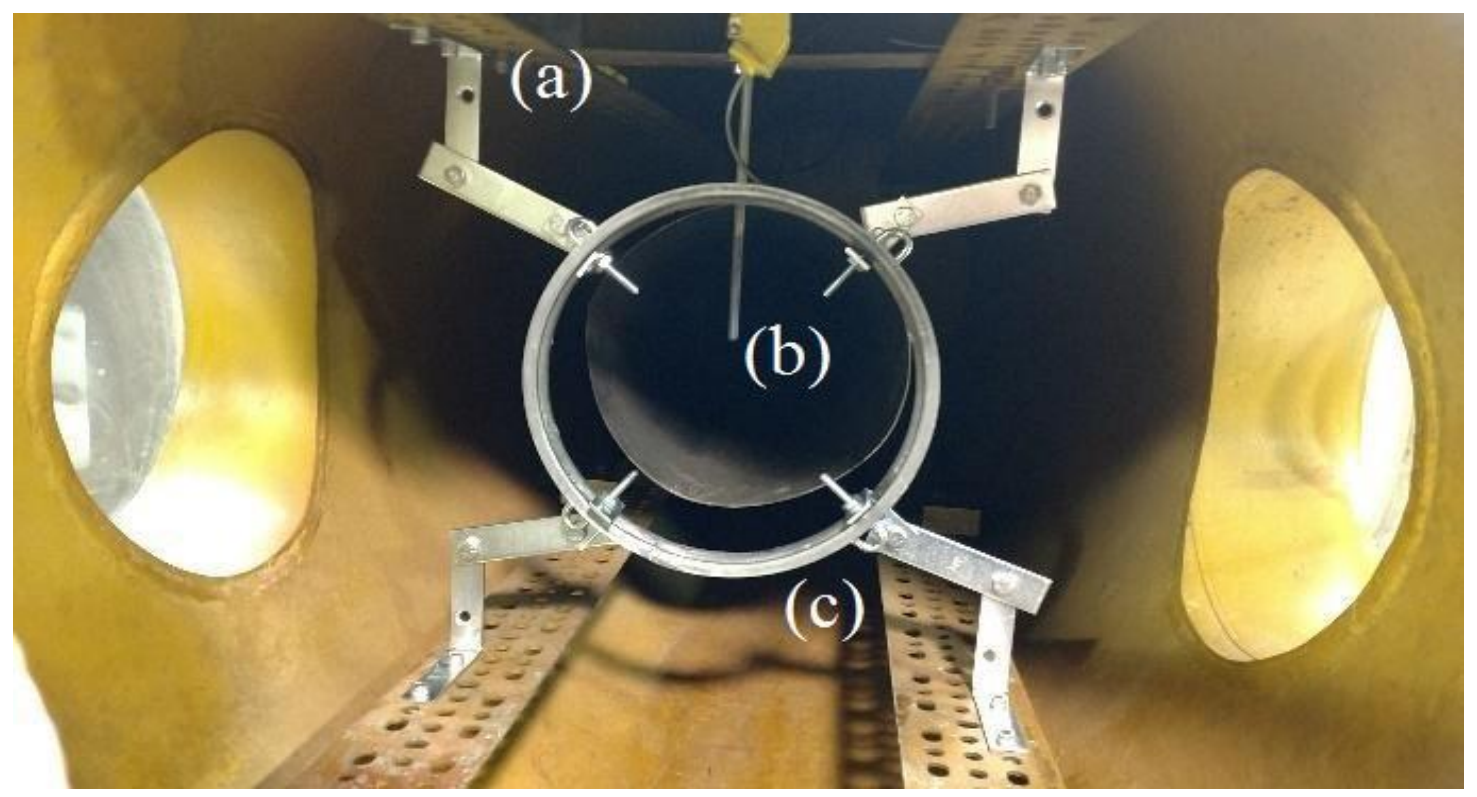

Figure 14: The instrumented toroid model mounted in the wind tunnel with positions shown for measuring Tinf (a), Tplume (b), and Tmod (c) 


\section{$\underline{\text { Results and Discussion }}$}

Thermal radiation encompasses a portion of the electromagnetic spectrum for wavelengths between approximately $0.1-100 \mu \mathrm{m}$ [21]. In its natural state argon gas is considered radiatively inert. That is, it neither absorbs nor emits thermal radiation with respect to its surroundings [21]. However, once ionized argon's emissivity increases dramatically and, under certain conditions, can function as a blackbody [20].

There are many factors that go into calculating the emissivity of a plasma. These include, but are not limited to, temperature, wavelength, radiation energy, and ambient pressure [20] [22]. The radiation energy emitted by the argon plasma can be calculated using the Stefan-Boltzmann law:

$$
E=\epsilon \sigma T^{4}
$$

For the purposes of this analysis the emissivity was derived from a figure included in Goldbach et al. [20] and reproduced in Figure 15.

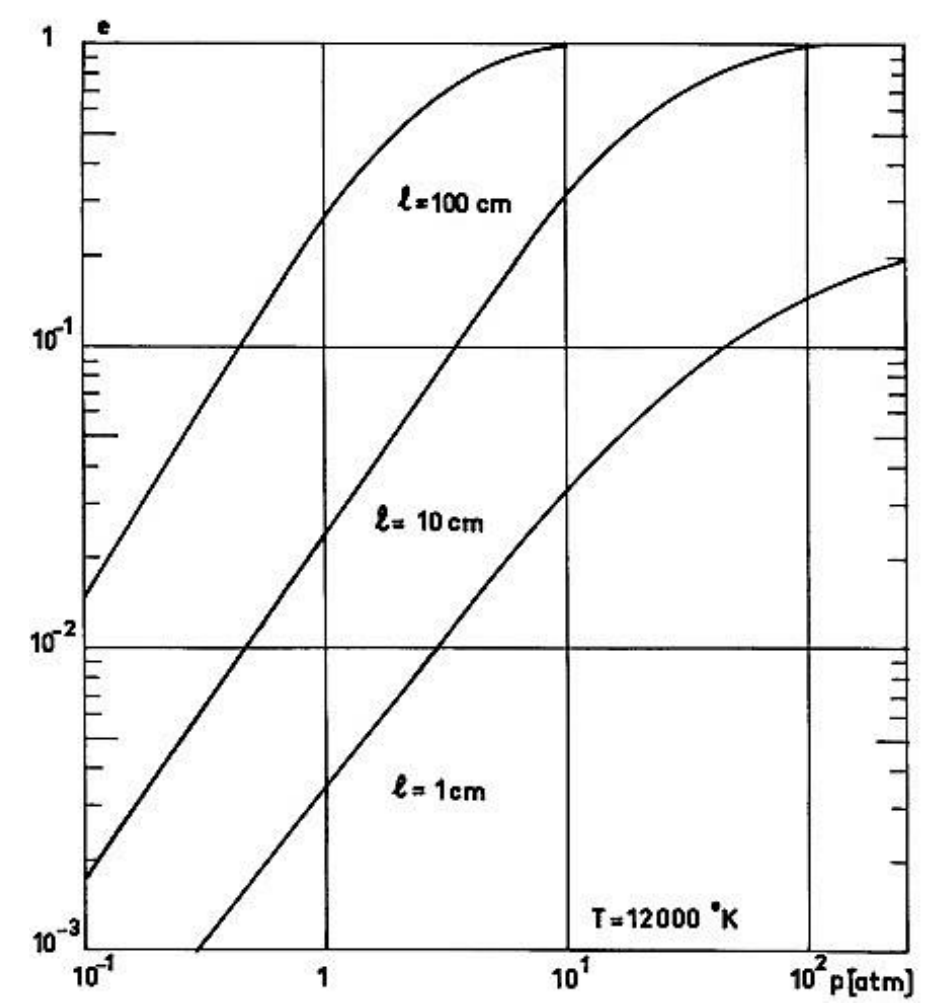

Figure 15: "The emissivity of an argon plasma at $\mathrm{T}=12000 \mathrm{~K}$ " [20]

Figure 16 and Figure 17 depict the radiation heat transfer effects on the toroid with and without magnets, respectively. 


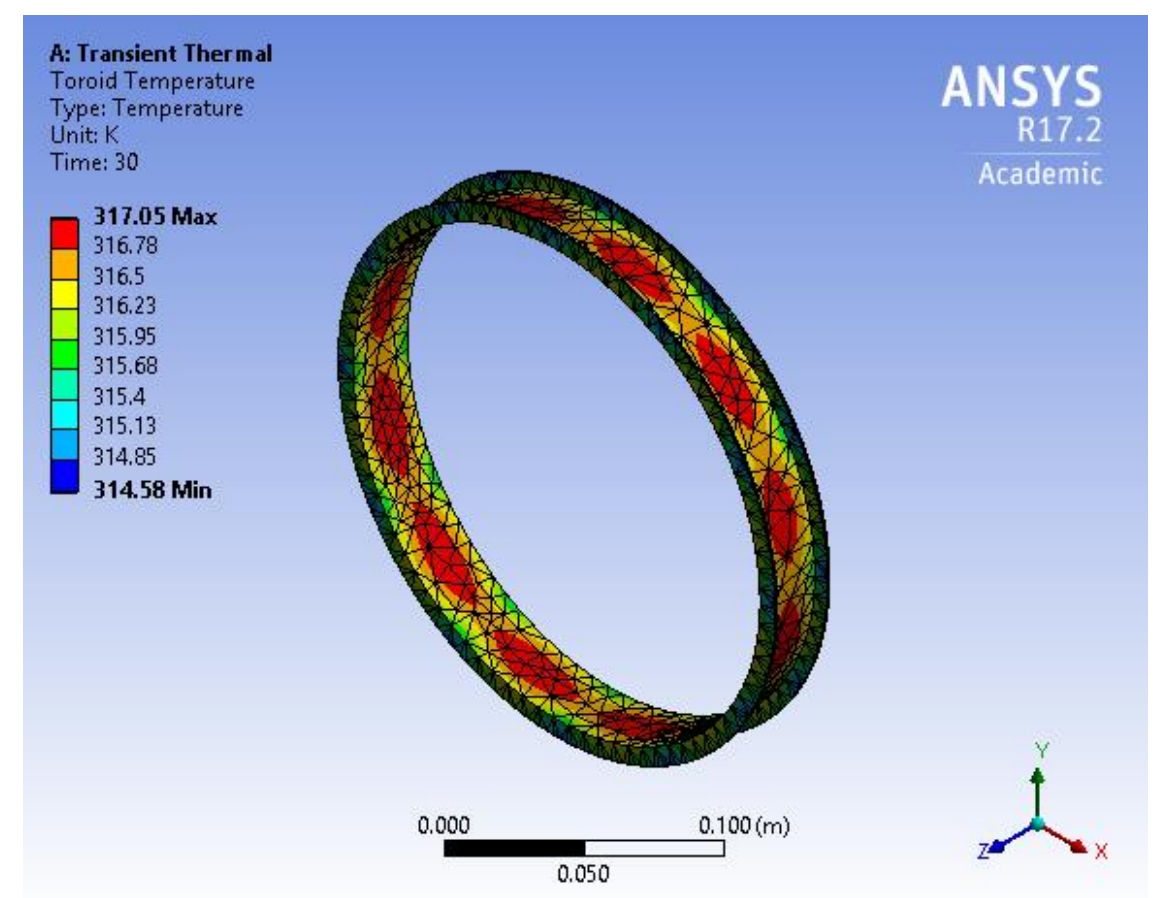

Figure 16: Toroid temperature profile with magnets

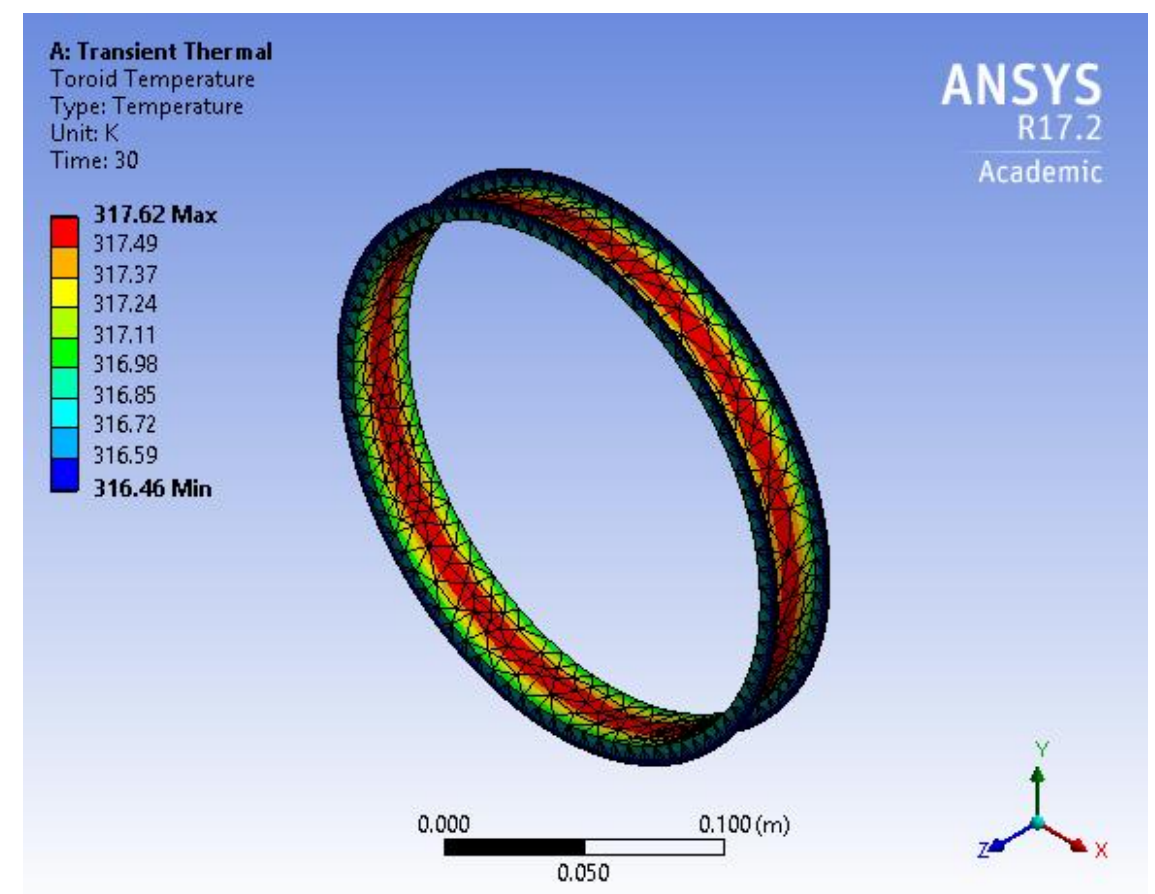

Figure 17: Toroid temperature profile without magnets

While thermal radiation accounts for most of the heat absorbed by the magnets, conduction heat transfer is equally as important for this analysis. Because the magnets are mounted on a steel toroid most of the radiation does not reach them directly. Instead, heat is first absorbed by the toroid and transferred to the magnets based on Fourier's law of heat conduction: 


$$
\dot{Q}_{\text {cond }}=-k A \frac{d T}{d x}
$$

where $d x$ indicates the direction heat travels in one dimension [21]. The conduction heat transfer effects on the toroid can clearly be seen by the temperature changes in Figure 16 as opposed to Figure 17.

It is clear from Figure 18 and Figure 19 that heat conduction between the magnets and the steel toroid primarily is predicted to take place where the flat ends of each magnet connect with the sides of the toroid.

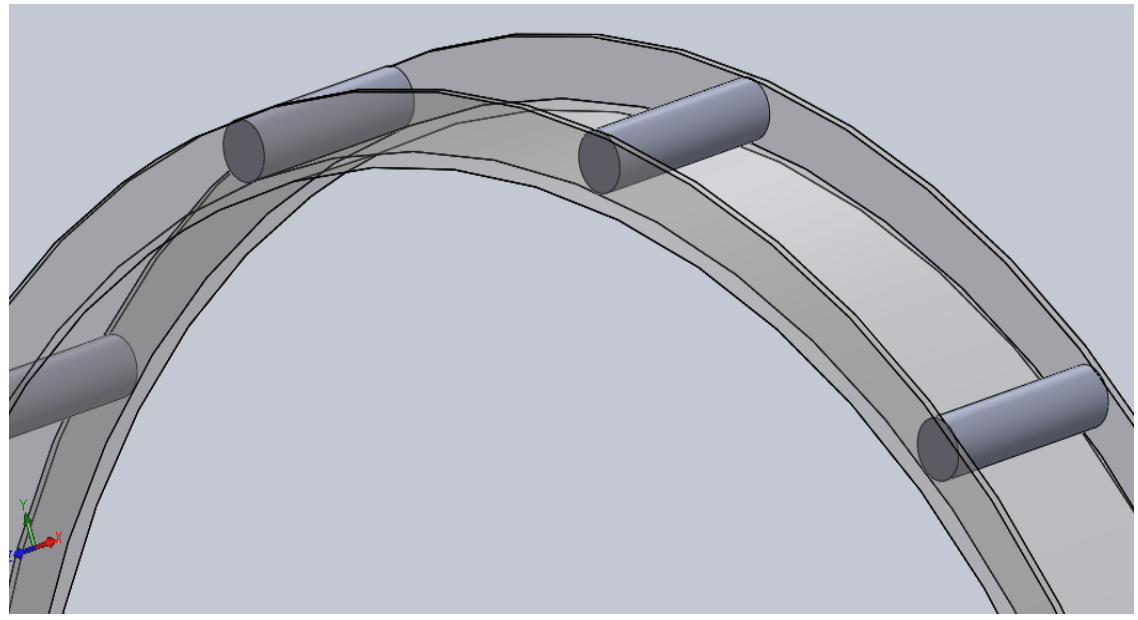

Figure 18: Magnet mounting positions assuming perfect abutment

It should be noted that some radiation heat transfer also takes place between the toroid surface and the section of each magnet directly facing it. However, the temperature difference due to this radiation is negligible compared to the conduction heat transfer between adjacent surfaces. Figure 18 shows the final temperature of the magnet after 30 seconds of heat exposure. 


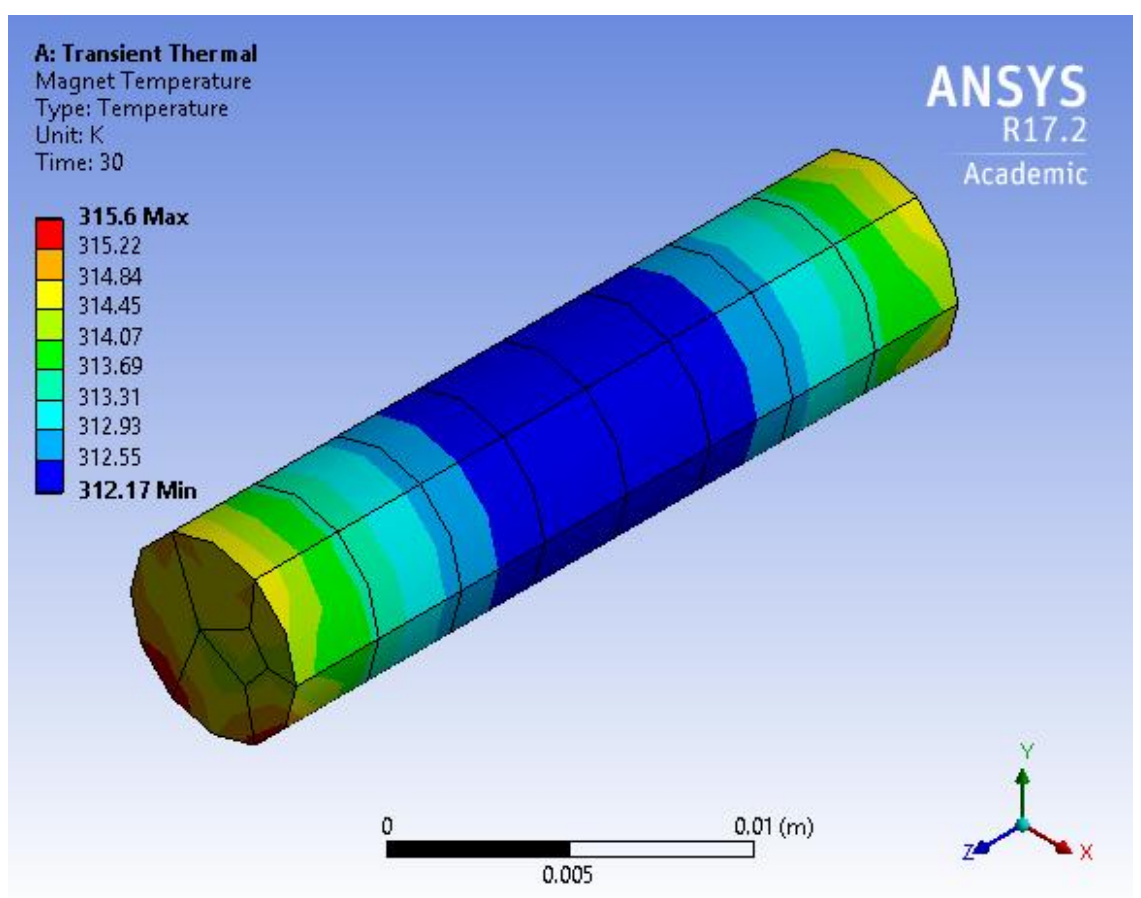

Figure 19: Magnet temperature profile

Comparing Figure 16 and Figure 19 reveals a measurable difference in temperature between the magnet and the surface of the toroid. By absorbing any direct radiation, the steel toroid also becomes a thermal insulator for the magnets. This insulating effect is accentuated by the limited amount of contact between the steel toroid and cylindrical magnets as seen in Figure 18.

After 30 seconds of exposure to a steady stream of argon plasma the maximum calculated temperature of the steel toroid and the maximum magnet temperature was approximately $317 \mathrm{~K}$ and $316 \mathrm{~K}$, respectively. Based on these results the thermal radiation emitted by a steady stream of argon plasma is not predicted to cause a significant enough temperature increase to reach the magnets' maximum operating temperature within the designated time span. Further analysis revealed that the magnets' maximum operating temperature would be reached after approximately 2.5 minutes of continuous exposure to the argon plasma stream. 


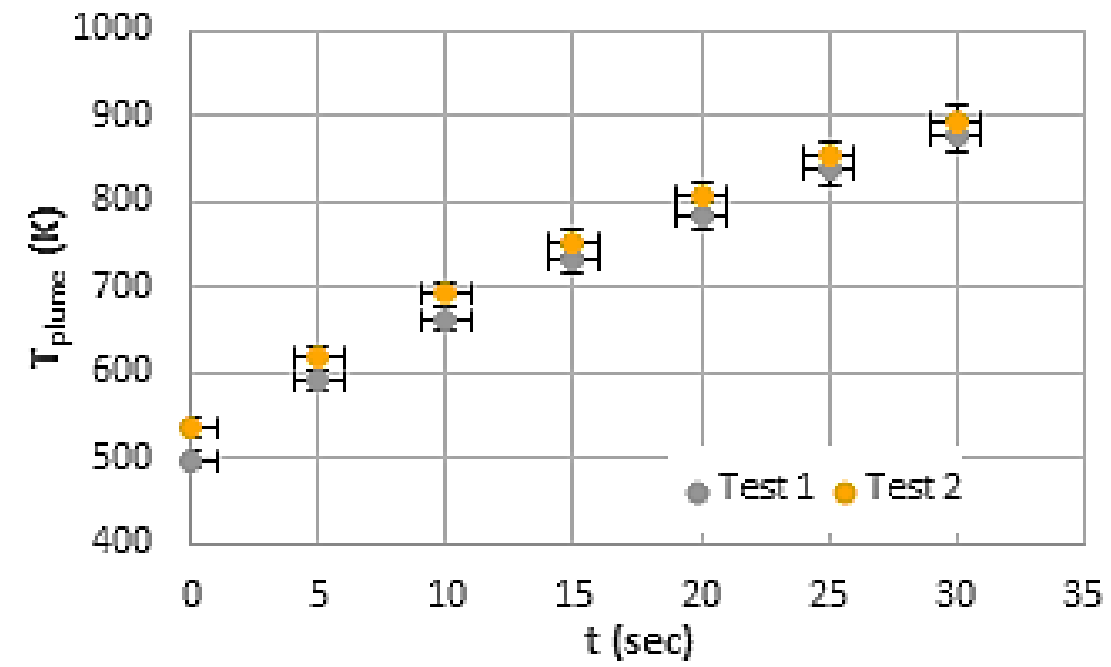

Figure 20: Time-variant experimental measurement of the plume temperature

Data accuracy was verified experimentally using the arc jet wind tunnel described above. A steel toroid without magnets was mounted within the tunnel and a series of temperature probes measured the active temperatures of both the toroid and the argon exhaust plume. Two representative sets from the experimental tests are presented in Figure 20 and Table 4.

Table 4: Experimental temperature values for infinitely, model and plume locations

\begin{tabular}{|c|c|c|c|c|c|c|}
\hline $\mathbf{t}(\mathbf{s e c})$ & $\mathbf{T}_{\text {inf }}(\mathbf{K})$ & $\mathbf{T}_{\text {plume }}(\mathbf{K})$ & $\mathbf{T}_{\text {mod }}(\mathbf{K})$ & $\mathbf{T}_{\text {inf }}(\mathbf{K})$ & $\mathbf{T}_{\text {plume }}(\mathbf{K})$ & $\mathbf{T}_{\text {mod }}(\mathbf{K})$ \\
\hline $\mathbf{0}$ & 292 & 499 & 306 & 291 & 537 & 312 \\
\hline $\mathbf{5}$ & $\sim$ & 591 & $\sim$ & $\sim$ & 618 & $\sim$ \\
\hline $\mathbf{1 0}$ & $\sim$ & 664 & $\sim$ & $\sim$ & 692 & $\sim$ \\
\hline $\mathbf{1 5}$ & $\sim$ & 733 & $\sim$ & $\sim$ & 752 & $\sim$ \\
\hline $\mathbf{2 0}$ & $\sim$ & 784 & $\sim$ & $\sim$ & 806 & $\sim$ \\
\hline $\mathbf{2 5}$ & $\sim$ & 837 & $\sim$ & $\sim$ & 852 & $\sim$ \\
\hline $\mathbf{3 0}$ & 292 & 877 & 314 & 291 & 892 & 320 \\
\hline $\mathbf{\Delta T}(\mathbf{K})$ & 0 & 378 & 8 & 0 & 355 & 8 \\
\hline
\end{tabular}

The thermocouple set to measure the test chamber temperature $\left(T_{\text {inf }}\right)$ varied less than $1 \mathrm{~K}$ in both tests. The thermocouple placed on the toroid showed a model temperature $\left(T_{m o d}\right)$ change of $8 \mathrm{~K}$ in both cases, even though the tests did not start with the same initial temperatures. Because the time and temperature measurements were taken manually, the uncertainty in the actual time of the measurement is estimated to be \pm 1 second. Within the temperature range tested, the maximum likely systematic uncertainty in the actual temperature measured from each probe is $2.1 \%$. It is worth noting that although the time constant for the large probe used to measure the temperature of the argon plasma plume ( $\left.T_{\text {plume }}\right)$ is an order of magnitude greater than the time constant for the 
smaller probes used, the influence of thermal inertia on readings from the large probe is negligible after the relatively long period of investigation. For example, using measurements taken from Test 2 it is possible to show that the difference between the measured and the estimated steady state temperature reading after 30 seconds is $15 \mathrm{~K}$. This argument can be extended to the smaller probes with even greater confidence due to the lower time constant associated with their smaller size and grounded construction. When compared with experimental data the maximum temperature from Figure 16 had a $0.89 \%$ error based on the equation:

$$
\% \text { Error }=\frac{\text { Measured } \text { Value }- \text { Accepted Value }}{\text { Accepted Value }} \times 100
$$

The radiation heat transfer effect of argon plasma on a series of neodymium magnets mounted on a magnetically conductive toroid was numerically and experimentally investigated. The results showed that, without convection heat transfer, a toroid positioned approximately $5 \mathrm{~cm}$ outside the plasma jet will not reach the magnet's maximum operating temperature within a 30 second time span. This is because the mounting toroid insulates the magnets by absorbing most of the direct radiation heat transfer and transferring it to the magnets convectively. This process decreases the effective heat transfer rate from the plume to the magnets, allowing for more exposure time. The decreased heat transfer rate allows the toroidal apparatus to run for approximately 2.5 minutes with continuous radiation exposure before reaching the magnet's maximum operating temperature. 


\section{Overcoming Challenges}

\section{Apparatus Design}

There were several design flaws within the apparatus which had to be reconciled prior to testing. Among these were the placement of spoke holes, removing excess material from the toroid, fastening the $\mathrm{J}$ bolts, and increasing the toroid width without causing adverse deformation.

While the internal diameter of the shopping cart wheel was consistent with design specifications of approximately $22 \mathrm{~cm}$, once the part was delivered several alterations were necessary. First, the rubber tire had to be removed. This was accomplished using a clamp and several screw drivers to increase leverage. Once the tire was gone the wheel spokes also had to be removed. Bolt cutters were used to remove the central axle and each spoke was pulled off by hand. However, this process left several holes in the toroidal rim. To avoid removing too much material larger holes were drilled through the existing ones to accommodate $\mathrm{J}$ bolt installation.

Upon completion of these alterations it became apparent that the remaining steel toroid was coated in paint. This coating was problematic because it decreased the size of the magnet housing area and prevented direct contact between the magnets and the toroid. Much of this coating was removed using a rotary tool and the remainder was removed using a combination of sandpaper and steel wool. It was determined that removing this excess material to allow direct contact between the magnets and toroid was important based on the numerical analysis displayed in Figure 10. This analysis indicated the strength of the magnetic field transferred between magnet and toroid would have decreased if the distance between them increased. Additionally, the calculations only accounted for the permeability of free space (i.e. air) so factoring in the unknown permeability of the coating may have had adverse effects on the total span of the magnetic field.

Although exposing the bare metal of the toroid did increase the width of the magnet housing area depicted in Figure 18, the area was still slightly smaller than the magnets. To further extend the toroid surface area the groove along the toroid's central plane, visible in Figure 7 and Figure 8, had to be flattened. The steel mounting was set on a flat surface and the groove was hammered out to avoid elongating the toroidal structure. Some elongation did occur but this was remedied by strategically fastening the shortest and longest ends to the mounting structure in Figure 8. The resulting structure had a diameter of $22 \mathrm{~cm}$ with a concentricity of $\pm 0.3 \mathrm{~cm}$ 


\section{Wind Tunnel Repairs}

After the wind tunnel was initially updated several repairs had to be performed because the arc jet operated incorrectly. Repairs included replacing several O-rings which melted when the arc jet was run for too long, mending one of the combination coolant/power lines, and replacing one of the copper fittings in the arc jet.

After running the arc jet successfully for the first time a coolant leak appeared through the exhaust nozzle in Figure 21.

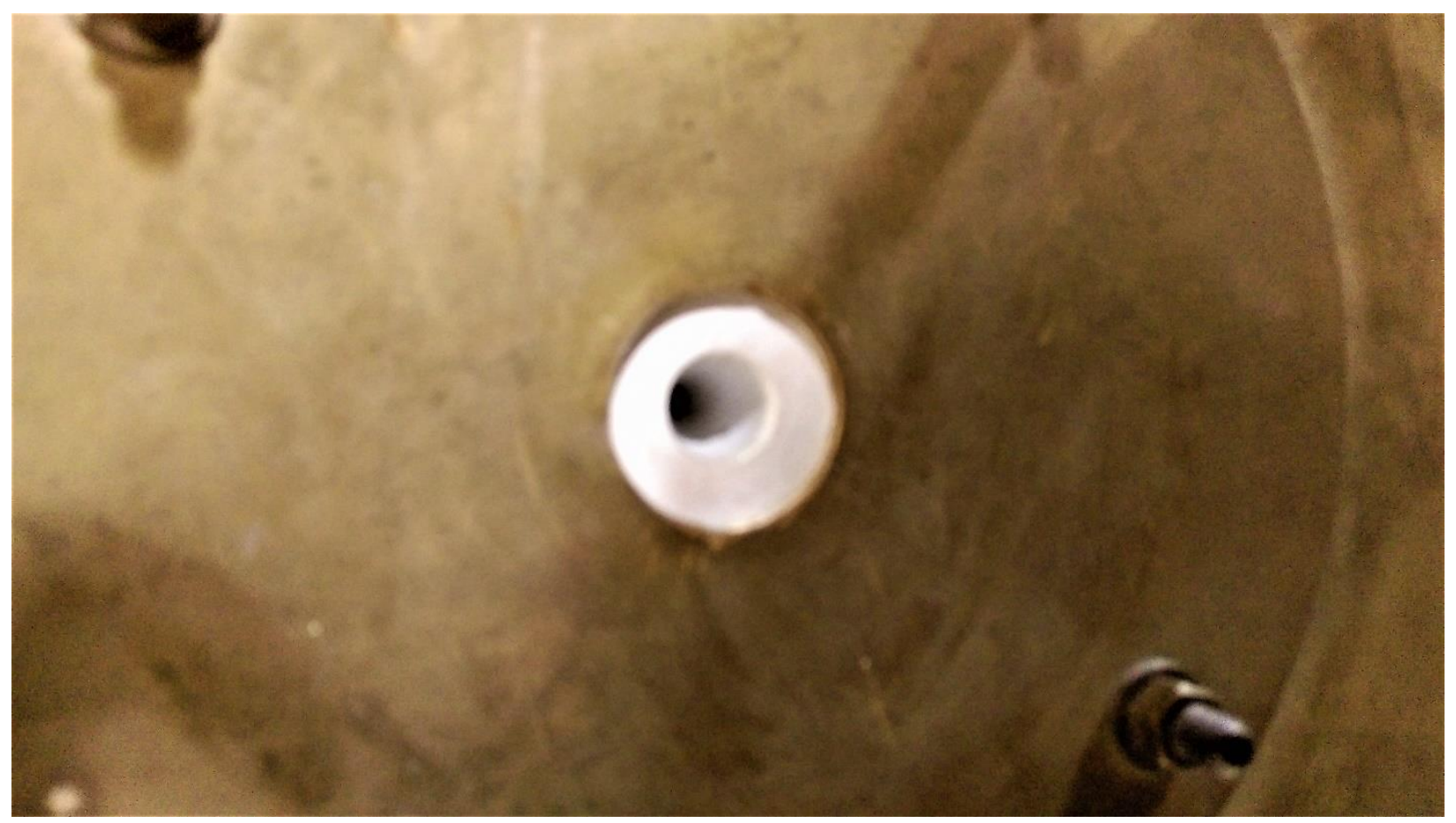

Figure 21: Arc jet exhaust nozzle

To diagnose this problem the arc jet had to be removed from the wind tunnel and dismantled as in Figure 4. It was determined that insufficient cooling caused several of the rubber O-rings to melt. O-rings of two different sizes had to be replaced. The larger O-rings had an outer diameter of $1^{1} / 8$ " $(2.86 \mathrm{~cm})$ and a thickness of ${ }^{1} / 8 "(0.32 \mathrm{~cm})$ while the smaller ones had an outer diameter of $11 / 16$ " $(2.70 \mathrm{~cm})$ and a thickness of $1 / 16 "(0.16 \mathrm{~cm})$. Thus, a pack of 25 larger and 50 smaller O-rings made from oil-resistant Buna-N rubber were purchased. The size of each order was not based on need but availability from the supplier. This process took several weeks during which the MATLAB portion of the numerical analysis was completed. These same O-rings had to be replaced multiple times until it was determined that the arc jet could not be run for longer than 30 seconds with a minimum of a three-minute cool down period between each run. 
A combination coolant/power line from the tunnel's high frequency DC starter to the arc jet's anode burst after some initial testing due to excessive heat exposure when the coolant pump was shut off prematurely. Excessive heat from the arc jet and power line caused the stagnant coolant remaining in the line to expand and vaporize. The vaporized coolant melted the rubber exterior forming a bubble thin enough to rupture. The line displayed in Figure 22 was subsequently repaired and refitted on the tunnel.

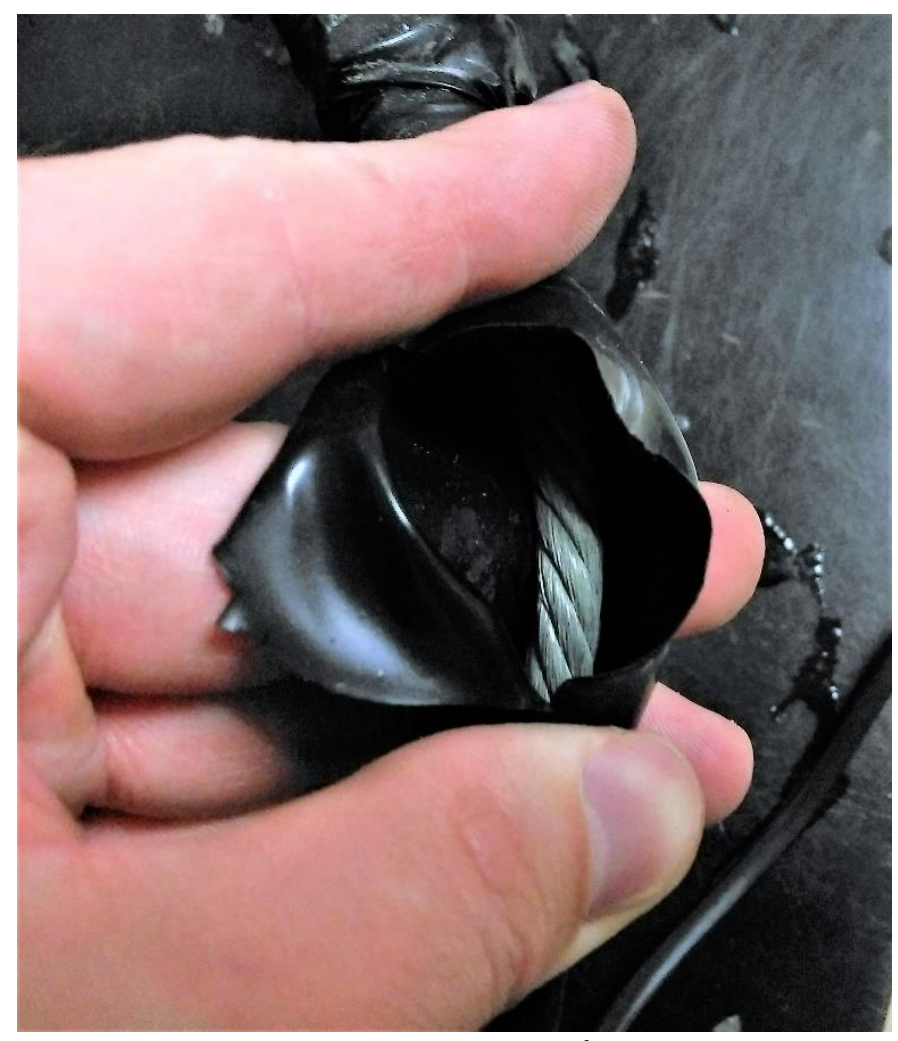

Figure 22: Ruptured coolant/power line

To repair the coolant/power line properly the weakened section had to be removed and the two remaining sections reattached. The severed ends of the metal rope were soldered together with a brass fitting equipped with enough room for coolant flow. The two ends of the line's rubber exterior were then clamped around the fitting and wrapped in electrical tape to prevent further leaking as depicted in Figure 23. The three-minute cool down period described above was implemented partially to prevent future ruptures. 


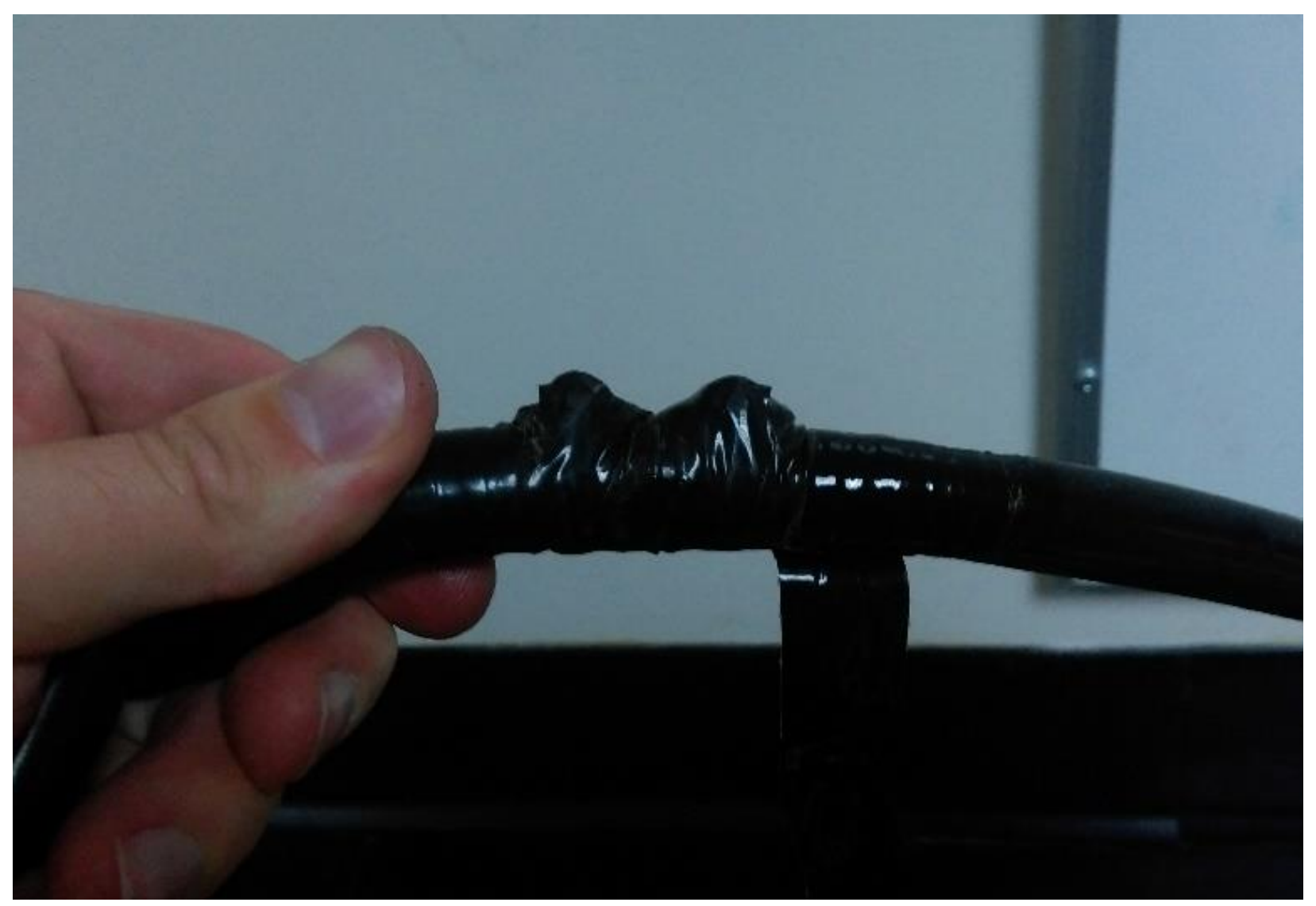

Figure 23: Repaired coolant/power line

While repairing the ruptured line took a significant amount of time, it also afforded a view into how the arc jet is powered. DC electricity from the power supply described in previous sections is fed through the metal rope shown in Figure 22. A steady coolant flow is passed through the line to prevent overheating and carry the electric current simultaneously. Power was supplied to both the cathode and anode in this fashion. 


\section{The Experiment}

\section{Experiment Metric}

Data was collected using one type- $\mathrm{K}$ thermocouple connected to a multichannel thermometer, a Nikon D5300 camera with an adjustable lens connected to a tripod, a second camera for recording gauge measurements, the toroidal test apparatus previously described, a laptop equipped with digital photography (Digicam Control) and image processing software (MATLAB), one size 300 cylinder of argon gas with two pressure gauges, an ambient temperature thermometer, and a barometer to measure atmospheric pressure.

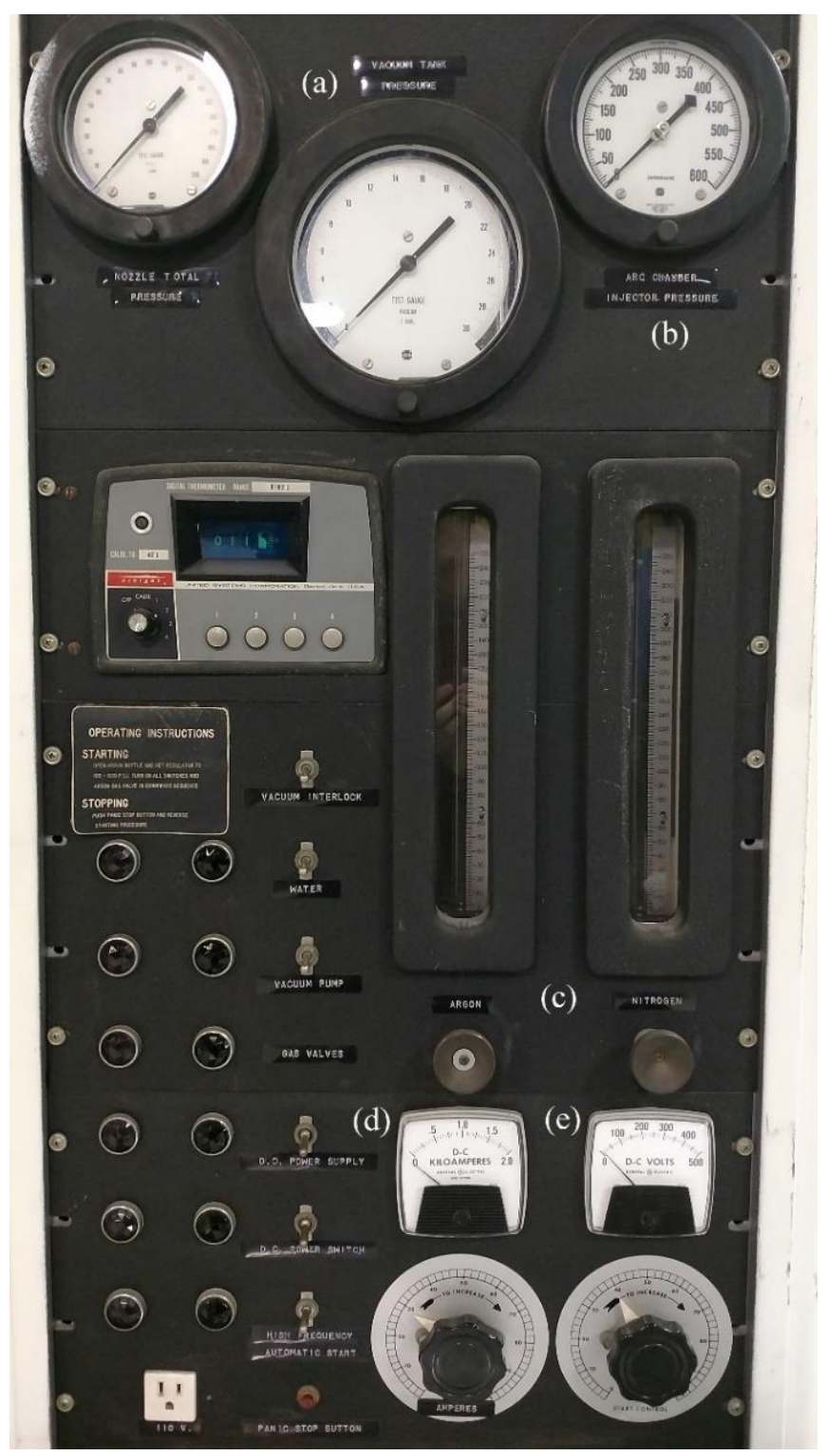

Figure 24: Wind tunnel control console with (a) vacuum pressure gauge, (b) injector pressure gauge, (c) flow meter, (d) ammeter, and (e) volt meter 
All equipment was calibrated and measurements have been adjusted for data accuracy. The temperature probe was calibrated between 273 and 373 Kelvin using the known melting and boiling temperatures of water. The probe measured $4 \mathrm{~K}$ above known values at both the melting point and boiling point. The barometer and ambient temperature thermometer were calibrated by comparing data with previously calibrated digital scales. The ambient temperature and pressure readings were determined to be accurate. Finally, the flow meter was calibrated using a water displacement method which confirmed measurement accuracy. All other equipment was previously calibrated and assumed to be accurate.

The wind tunnel control console from Figure 24 also contains a vacuum pressure gauge (a), an injector pressure gauge (b), a flow meter (c), an ammeter (d), and a volt meter (e). The Nikon camera used an adjustable 18-55 mm lens. For this experiment the camera's aperture was set at 4.5 , the ISO was 100 , and the shutter speed was $1 / 160 \mathrm{sec}$. The operating conditions for the wind tunnel are displayed in Table 5.

Table 5: Hypersonic wind tunnel operating conditions

\begin{tabular}{|l|c|}
\hline \multicolumn{1}{|c|}{ Operating Condition } & Value \\
\hline Argon Regulator Pressure [kPa (psig)] & $308.20(40)$ \\
\hline Argon Mass Flow Rate [SLPM] & 34.52 \\
\hline Average Vacuum Chamber Pressure [mmHg] & 2 \\
\hline Voltage [v] & 400 \\
\hline Current [amp] & 300 \\
\hline Electric Start Capacity [\%] & 50 \\
\hline
\end{tabular}

\section{Experimental Procedure}

The purpose of the procedure was to obtain photographic evidence of how an argon plasma jet interacts with a toroidal magnetic field.

\section{Setup}

First, all the materials had to be collected. The thermocouple was connected to the multichannel thermometer which was plugged into an external power supply as depicted in Figure 25. 


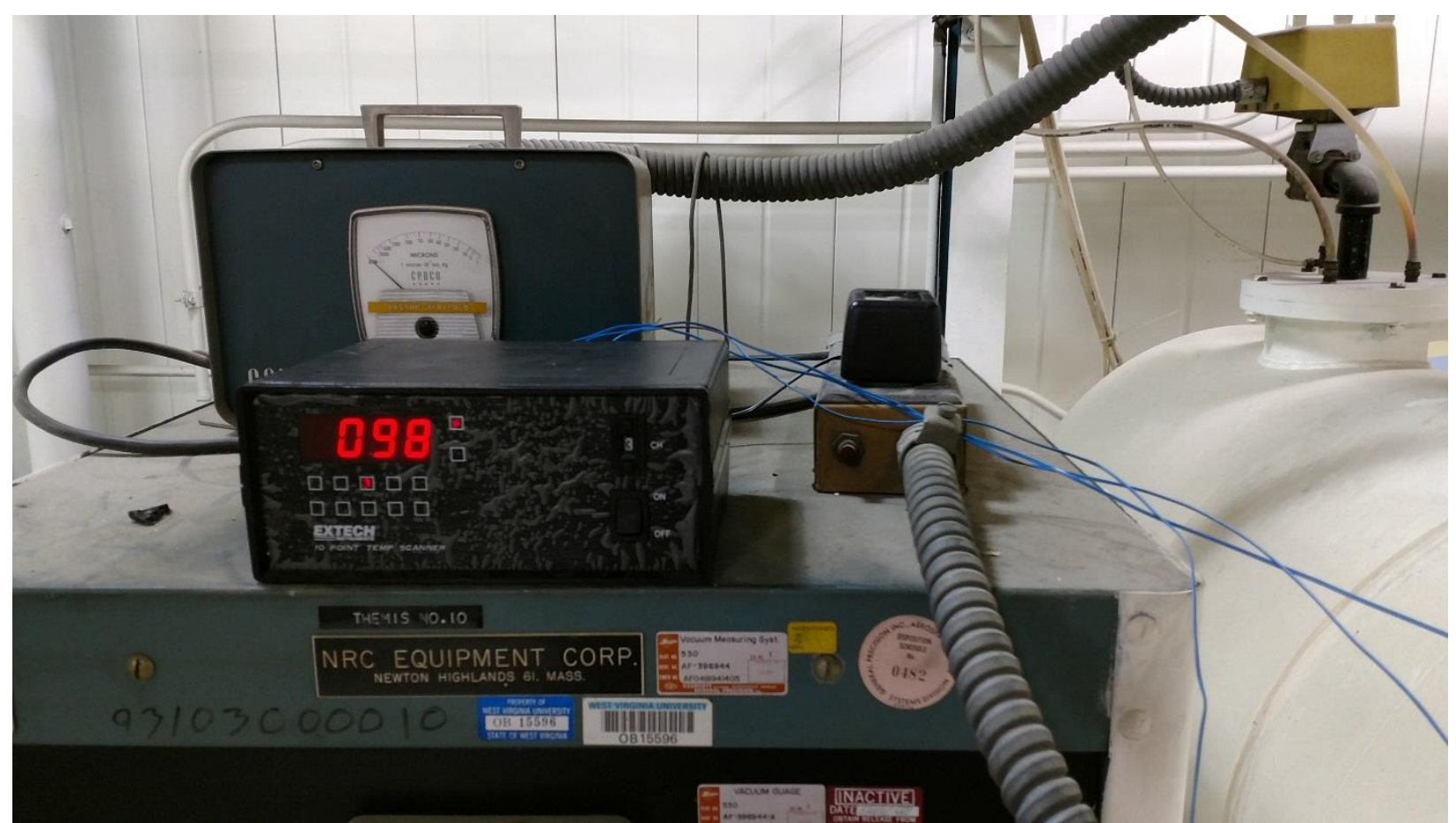

Figure 25: Multichannel thermometer connected to thermocouples located inside the vacuum chamber

One end of the pressure gauge was connected to the release valve of the argon cylinder as depicted in Figure 26. The other end of the pressure gauge was connected to plastic piping leading to the flow meters within the wind tunnel's control consol.

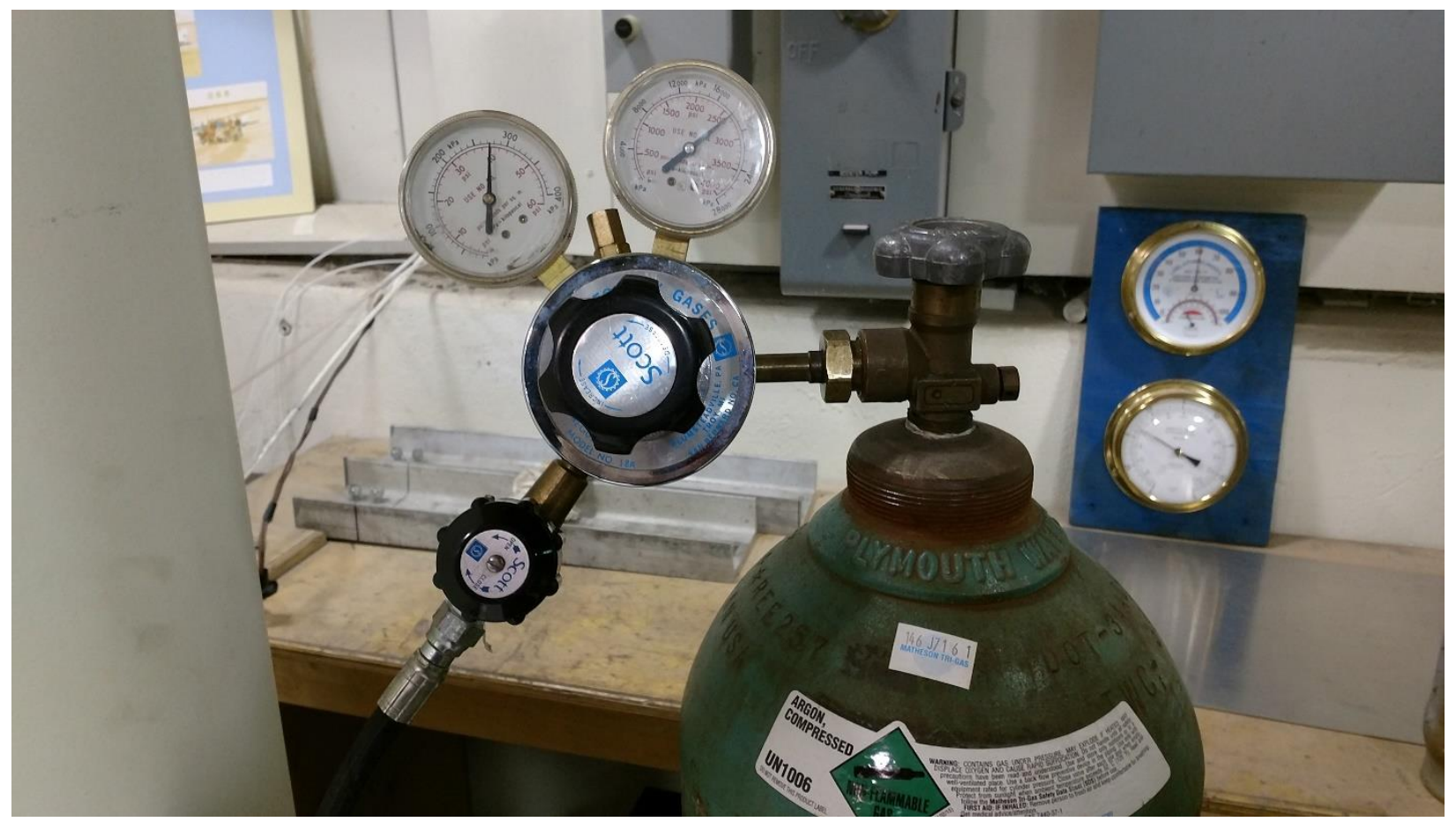

Figure 26: Pressure gauge connected to the argon cylinder 
A tripod was erected and attached to the camera so it faced one of the viewing ports within the hypersonic wind tunnel. The other viewing port was covered with an opaque fabric to prevent optical interference. The laptop was turned on and connected to the Nikon camera via a USB line. An external battery pack connected the camera to a power outlet. After starting the laptop, a program called Digicam Control was activated so the camera settings could be manipulated remotely through the USB connection. The fully constructed camera equipment setup is depicted in Figure 27.

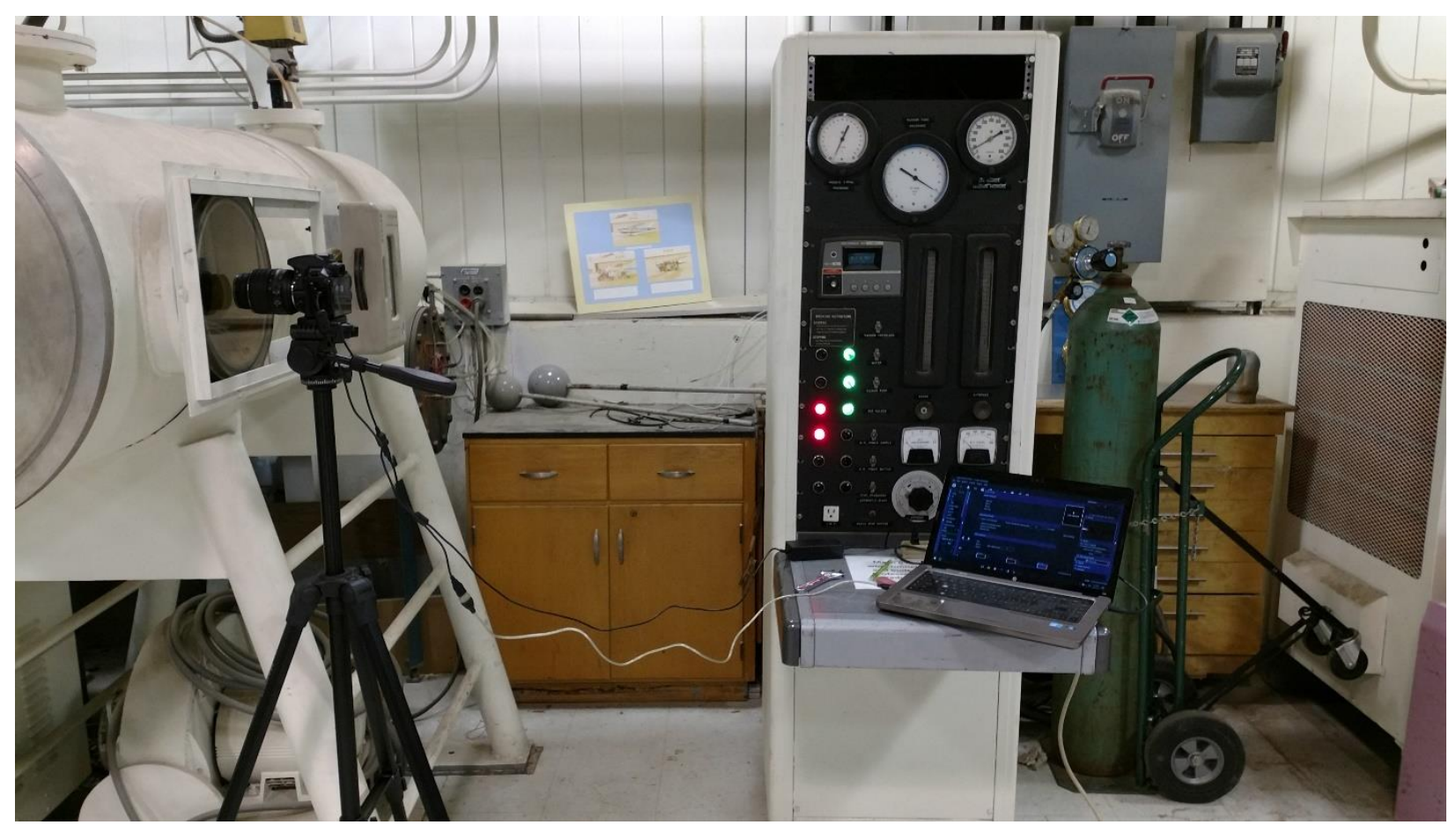

Figure 27: Fully constructed image collection system

The final step in setting up the full apparatus was to install the magnetic field configuration for each test.

Before any testing could begin the Nikon camera lens needed to be properly focused for optimal image clarity. To do this a ruler was positioned in the wind tunnel near the central location of the exhaust plume. The ruler was mounted on several wooden blocks and supported by mounds of clay so the numbers were clearly visible as depicted in Figure 28. 


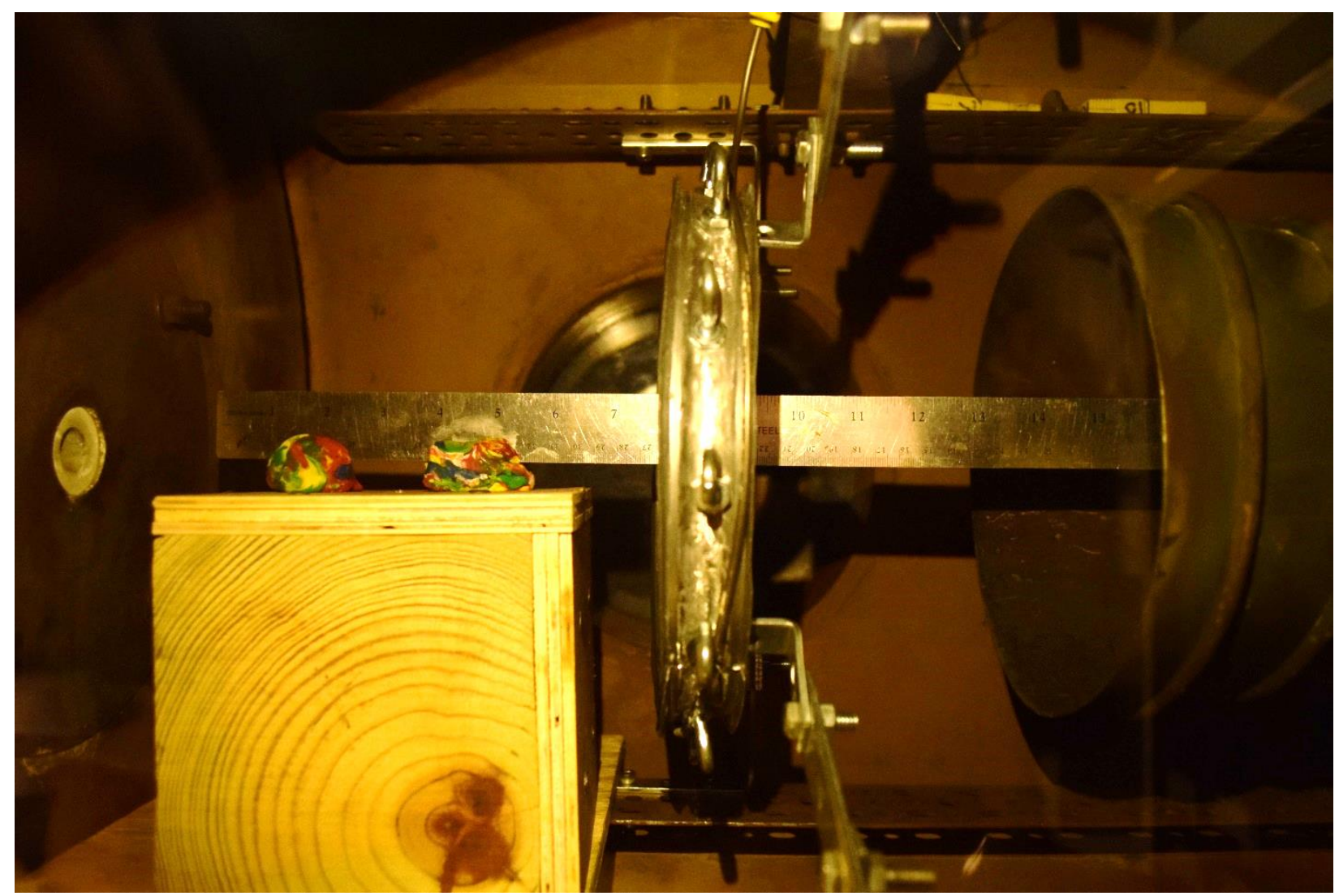

Figure 28: Apparatus used to focus the camera before data collection

The digital zoom was maximized through the laptop and the camera lens was adjusted manually to focus on the ruler. Adjusting the camera settings in this way ensured an accurate point of focus within the argon plume allowing for a clearer image. Once the camera was properly focused it could not be moved for any reason until all data was collected. Altering the camera's focus or physical position by any amount would skew any results from the image processing techniques described in later sections. This is partially why images were captured remotely rather than using the physical shutter button on the camera.

\section{Testing}

The first step in each test was to activate the vacuum interlock, start the cooling system, and start the duel stage vacuum pump and roots blower system. The duel stage system had to be run for a minimum of 10 minutes before the vacuum chamber reached its optimal pressure from Table 5. During this time the computer program controlling the Nikon camera was set to capture four images per test. Photographs were taken 10 seconds apart with the first image being captured 10 seconds after the digital shutter button was clicked on the computer screen. After the necessary vacuum was reached the argon cylinder was opened and the output pressure gauge was set to 40 
psig (308.20 kPa). The flow meter depicted in Figure 29 was set at $100 \mathrm{~mm}$ which converts to a flow rate of 34.52 SLPM [23] and images of all gauge measurements were recorded photographically using the second camera.

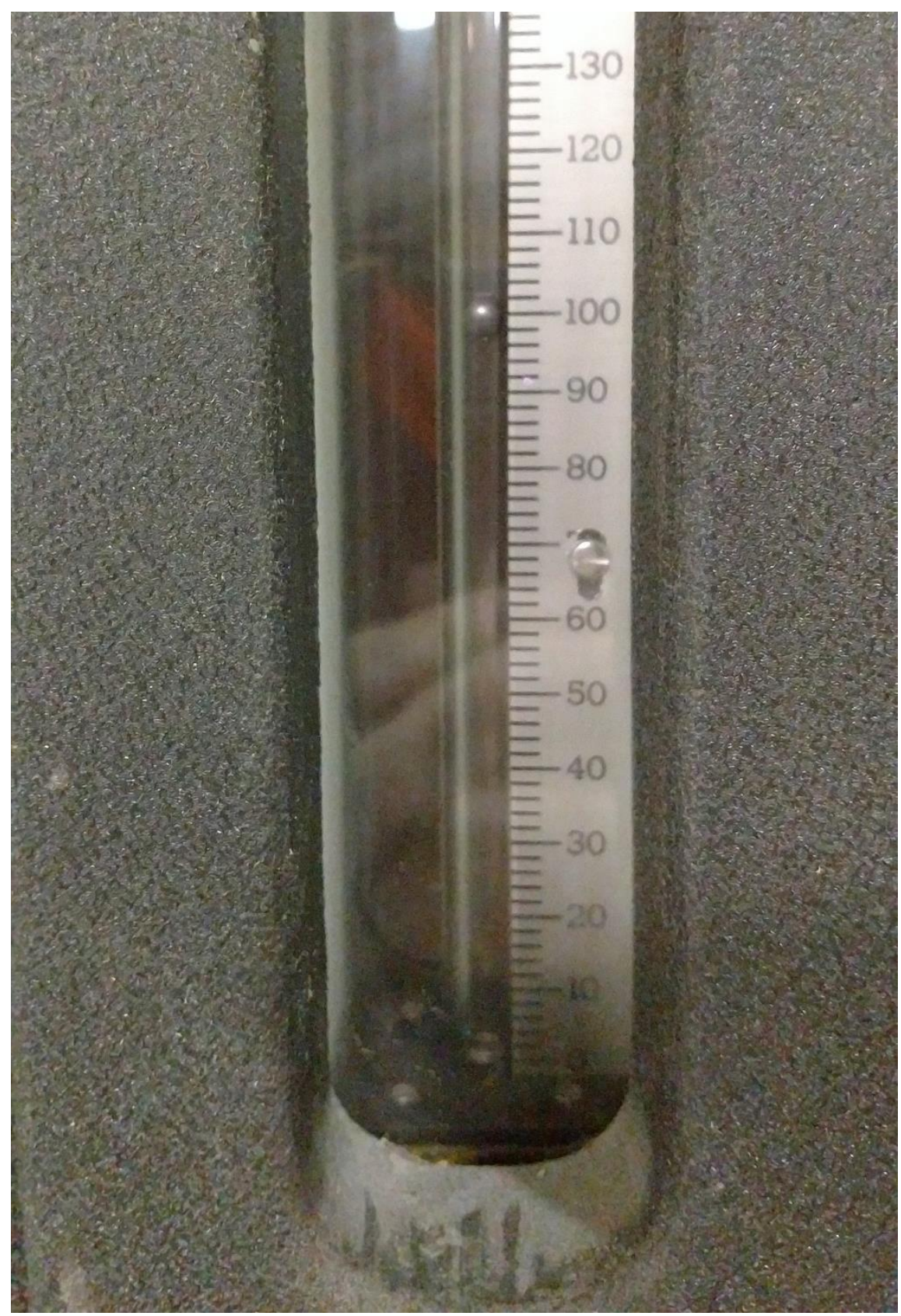

Figure 29: Flow meter set at $100 \mathrm{~mm}$

At various times throughout the testing process the ambient pressure changed and was photographically recorded. When the ambient pressure changed the time and most recent magnetic field orientation was also recorded. The total vacuum pressure was calculated by subtracting values from the vacuum pressure gage and the ambient pressure gage. The total vacuum pressure needed 
to be recorded accurately, within two decimal places, for each test because having a different vacuum pressure could affect the exhaust profile for a given test and potentially skew the experimental results.

To eliminate any potential glaring in the wind tunnel only one overhead light in the room was left on after the gauge measurements were recorded. The DC power supply and DC power switch were activated on the wind tunnel's control console and the starting voltage for each test was set at 400 volts. Once the power supply was activated the test was ready to begin. To initiate each testing sequence the electronic shutter button was triggered on the laptop. The wind tunnel's automatic starter was activated immediately after the first image was taken. This was done to ensure images were consistently recorded 10, 20, and 30 seconds after the arc jet was activated. The automatic stop button was pressed immediately after the fourth picture was taken to prevent the arc jet from overheating.

Each testing sequence contained six runs for a single magnet orientation. The toroid temperature was recorded before and after each run. To ensure the magnets did not reach their maximum operating temperature a 5-10 minute cool down period was observed between tests. The starting temperature for any given test was between $80-100{ }^{\circ} \mathrm{F}(300-311 \mathrm{~K})$. This period also prevented the arc jet from overheating due to the temperature build-up from each test. The cool down time between each test was also recorded. After each sequence an additional five-minute cool down period was observed before closing the argon release valve and shutting down the wind tunnel completely. Once the vacuum interlock was deactivated the wind tunnel was opened and the magnet orientation was changed to begin another sequence. After each sequence, all photographs were collected into a single folder which was titled based on the magnet orientation of that sequence. After all sequences were completed and all data was recorded the testing equipment was disassembled and stored per the mandated requirements. 


\section{Results}

\section{Data Set 1}

Measurements for this experiment were collected in two distinct data sets. For the first data set, three magnet orientations were tested. The first sequence contained a control test without magnets, but included the toroid (toroid test). The second sequence had magnets arranged parallel to the toroid's central axis with the magnets' north poles facing the exhaust nozzle (axial north test). The third sequence was identical to the second, except the magnets' south poles were facing the exhaust nozzle (axial south test). During these testing cycles the argon flow was continuous to avoid any discrepancies in pressure and flow rate.

\section{Post Processing}

The first step in post processing this data was to write a MATLAB code that would compile a set of images and convert them to grayscale. Images for each analysis were uploaded manually based on the operating condition that was being studied. Several versions of this code were developed to study different operating conditions. From there the images were converted to binary form using a thresholding technique. The binarized images were processed by the computer as matrices with white pixels having a value of one and black pixels having a value of zero. These matrices were averaged together to produce a single image with various shades of gray. In these pictures, lighter regions indicated areas where the exhaust plumes from many images occupied the same space. The darker regions in these pictures indicated areas containing only a few exhaust plumes. An image like this was created for each time step in all three sequences. Appendix B contains each image developed for data set 1 . The MATLAB code for this analysis can be found in Appendix C

In analyzing these images several things became apparent. First, the exhaust plume expansion appeared to be time variant for each sequence. That is, the size of the exhaust plume decreased as time increased. The toroid test had the largest exhaust plumes, while the axial south test had the smallest exhaust plumes. Figure 30 and Figure 31 show that for the axial north and axial south tests the average 30 second plume was less than or equal to the size of the 20 second plume from the previous test. 


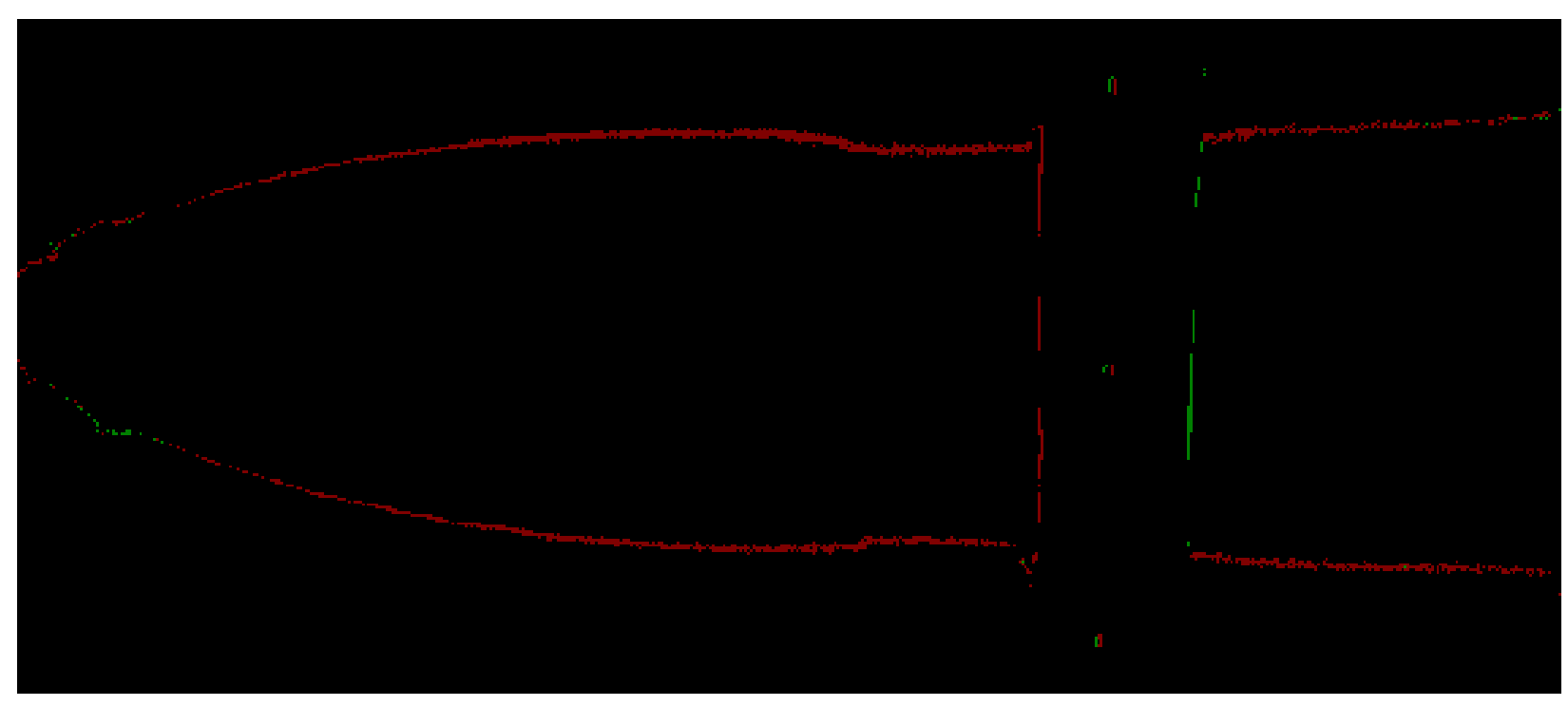

Figure 30: Toroid test (30 sec.) vs. axial north test (20 sec.)

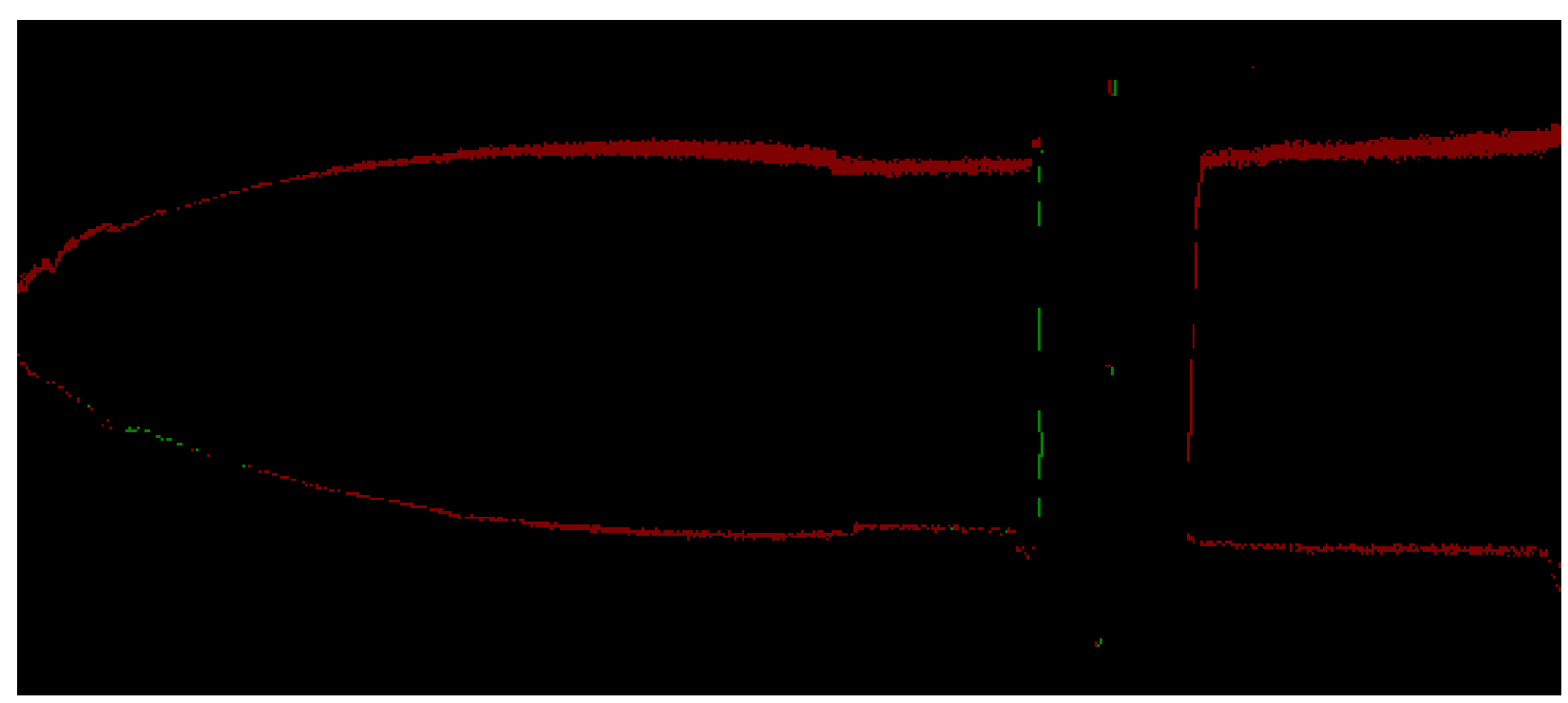

Figure 31: Axial north test (20 sec.) vs. axial south test ( $30 \mathrm{sec}$.)

Additionally, Figure 32 shows that the 30 second plume from the axial south test was even smaller than the 10 second plume from the toroid test. 


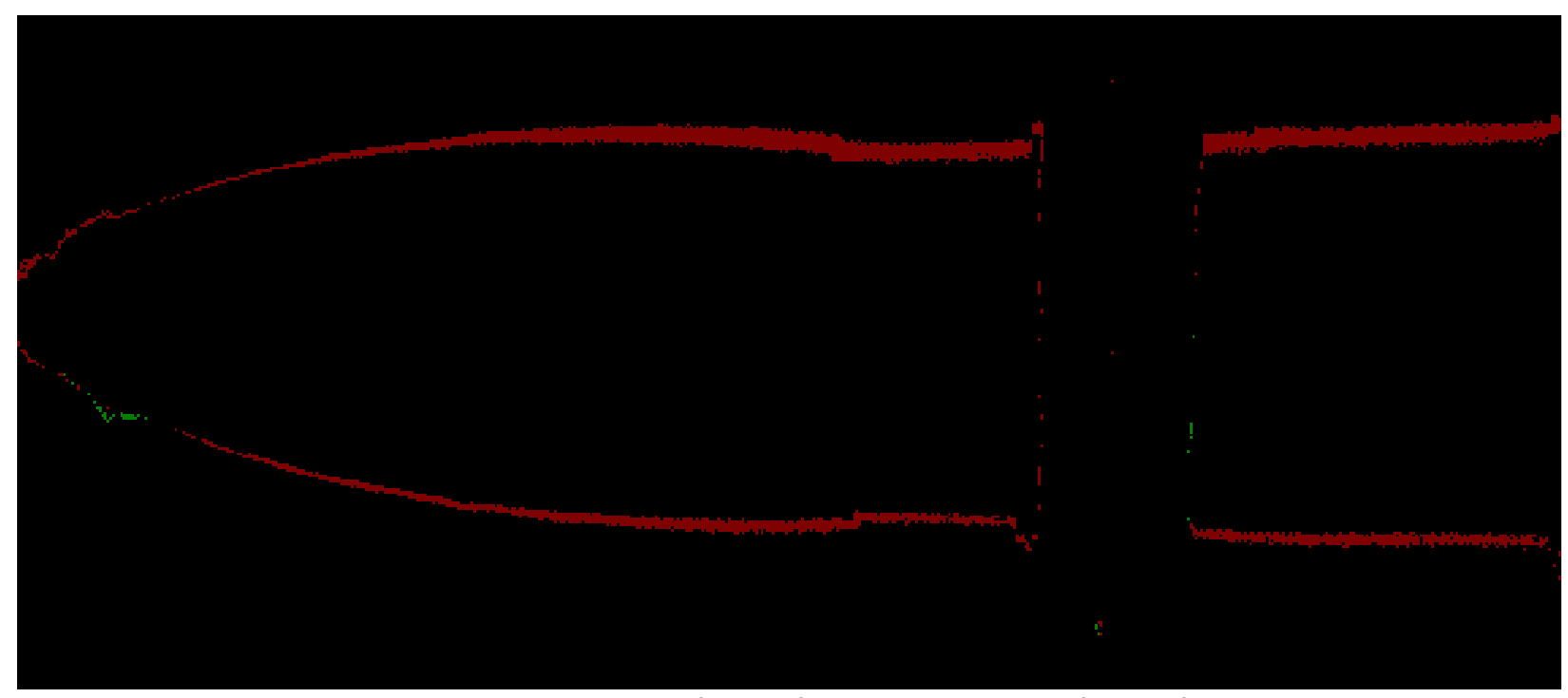

Figure 32: Toroid test (10 sec.) vs. axial south test (30 sec.)

These images were created using image processing techniques developed when post processing data set two.

\section{Data Set 2}

After performing an image analysis for the first data set several problems were identified. The primary concern was that the size of the exhaust plume decreased in the same order in which the experiments were performed. Given that an entire cylinder of argon was used throughout the testing process, due to the previously described continuous flow parameter, there was a concern that this phenomenon may have been caused by the decreasing pressure in the argon cylinder rather than the different magnetic field configurations. To alleviate this potential problem, it was determined that a second set of tests were necessary.

A new argon cylinder had to be purchased before testing could continue. There was an additional concern that one extra cylinder would not be sufficient. To remedy the situation the continuous flow parameter was replaced by utilizing a butterfly valve to turn the gas flow on or off without significantly altering the cylinder output pressure or flow rate during experimentation. The rate at which the valve was turned sometimes had a slight impact on the designated 40 psig $(308.20 \mathrm{kPa})$ pressure. In these cases, the cylinder output pressure valve was adjusted slightly to maintain a constant pressure between runs.

Another concern was that too few data points existed for each configuration. It was determined that the number of tests in each sequence should be doubled to twelve rather than six. In addition to the three existing configurations two additional magnet arrangements were added in 
data set two. In these configurations, the magnets were lined up orthogonally to the flow direction with either the north or south poles oriented in a clockwise direction (tangential tests). An additional control test was also run with no magnets and no toroid (control test) to determine what affects the toroid alone would have on the exhaust profile.

As previously described, changes in atmospheric pressure could negatively affect the experimental results by altering the vacuum pressure within the wind tunnel. Performing all tests in a single 24-hour period reduced the chances of having a significant variation in atmospheric pressure during each data set. However, in data set one it was assumed that the atmospheric pressure was constant throughout the day. In data set two, it became apparent that the atmospheric pressure did change slightly as the day progressed. A statistical solution to this problem was enacted by collecting two sets of six data points for each orientation at different times throughout the day.

\section{Post Processing}

Although the MATLAB code developed for data set one produced tangible results, the code was not as useful for data set two. The images produced for data set two were not as clearly defined as those from data set one. Consequently, the code had to be modified to become more robust. Rather than displaying the gray areas where not all elements in the matrix were either zero or one the code was modified so any value of 0.5 or higher was rounded to one, while all other values became zero. This produced a binarized image from the averaged images previously used. These new binary images were compared to the control tests described above with and without the steel toroid. To make these comparisons easier to understand regions where the flow affected by a magnetic field were larger than the control appeared in green, while regions where the flow affected by the magnetic field were smaller than the control appeared in red. This new code can be found in Appendix D, Appendix E, and Appendix F

The updated code was more robust, but required additional refinement to perform calculations in a timely manner. Adding more images and color comparisons significantly increased the time requirements of the data processing. This was problematic because the code had to be run multiple times to produce different comparisons. The size of the original images was uniformly reduced by approximately $33 \%$ to make the corresponding matrices smaller without altering the results. This was done by eliminating rows and columns within the image matrix that did not contain information pertinent to the analysis. That is, any large regions where no change 
occurred between images or regions containing large amounts of noise were cropped out. These smaller matrices took less time for computer processing. Additionally, different functions were used to process the images making the code more efficient. Images developed with this updated code can be found in Appendix G and Appendix $\mathrm{H}$ 


\section{Discussion}

The first step in analyzing the experimental results was to compare the data compiled from each orientation and time step to the control test. In comparing these images two things became immediately apparent. First, the toroid test and the tangential tests were similar to one another at every time step. This was especially true when compared against data with axial orientations.

The second observation was the appearance of a uniform size decrease in the exhaust plume across the bottom of all images when a comparison was made against the control test. This size adjustment resulted from the presence of a toroidal structure alone and had nothing to do with the magnetic fields. A slight increase in the top of the plume indicated that the flow altered its trajectory. This new trajectory brought the flow away from the bottom of the toroid and closer to the magnetic field at the top of the toroid. For this reason, the control test comparisons in Appendix $G$ only describe changes occurring at the top of each exhaust plume. Because the top of the exhaust flow was closer to the toroidal magnetic field it can be assumed that analyzing only the top half of each image effectively simulated conditions where the whole toroid had a stronger magnetic field. This assumption is supported by the exponential change in magnetic field strength depicted in Figure 10. Considering these observations, the next step in analyzing experimental results was to compare each magnet orientation directly to the toroid test. The results from both sets of analyses are described in the sections below.

\section{Comparison to Control Test}

The subsections below describe a comparative analysis of the exhaust profiles produced by different test apparatus configurations with respect to the control test. Descriptions of each exhaust profile were determined using a combination of the trends indicated in Table 6 and false color images displayed in Appendix G. Table 6 describes trends seen in this analysis in terms of the total expansion or reduction in the exhaust profile. The negative numbers in Table 6 indicate how much smaller a given exhaust profile was compared to the control test in terms of square centimeters. It is important to note that, due to the previously explained alteration in exhaust trajectory, the values in Table 6 were only used for the purpose of identifying trends in the exhaust profiles. 
Table 6: Exhaust plume percent decrease from the control test

\begin{tabular}{|c|c|c|c|}
\hline Control vs. Toroid Test & $\mathbf{1 0}$ seconds & $\mathbf{2 0}$ seconds & $\mathbf{3 0}$ seconds \\
\hline Control vs. Tangential North Test & -4.98 & -3.44 & -4.42 \\
\hline Control vs. Tangential South Test & -3.73 & -3.56 & -4.39 \\
\hline Control vs. Axial Test & -1.82 & -2.92 & -3.94 \\
\hline
\end{tabular}

\section{$\underline{\text { Transient Results }}$}

For the comparison between the toroid test and the control test, the 10 second and 30 second images yielded similar results where the plume affected by the toroidal structure was uniformly smaller than the control plume. However, in the 20 second time step the exhaust plume was only smaller in areas immediately in front of, and behind the toroid. See Figure 42, Figure 43, and Figure 44 in Appendix $\mathrm{G}$ for a visual comparison of each time step.

The next magnet orientation studied was a comparison between the control test and the magnet orientation where the north pole was oriented in a clockwise direction tangentially to the exhaust flow direction (tangential north test). These results showed that the exhaust plume at 30 seconds was smaller than the other two time steps. The 10 and 20 second time steps both decreased in size as they approached the toroid. However, at 20 seconds the size decreased less than the other two time steps making it the largest overall plume in this configuration. See Figure 45, Figure 46, and Figure 47 in Appendix G for a visual comparison of each time step.

The third magnet orientation studied was a comparison between the control test and the magnet orientation where the south pole was oriented in a clockwise direction tangential to the exhaust flow direction (tangential south test). The flow at the 10 and 30 second time steps started at about the same width as the control and narrowed as they approached the toroid. The 20 second time step started out slightly larger than the control but decreased in size immediately in front of and behind the toroid. In contrast to the first two orientations the flow in the 20 and 30 second time steps for this magnetic field actually became larger than the control shortly after passing through the magnetic field. See Figure 48, Figure 49, and Figure 50 in Appendix G for a visual comparison of each time step.

Data for the axial tests were nearly identical. As such, the results will be discussed together. For this data, the initial and final profiles were larger than the control profile at every time step. However, in the regions immediately in front of and behind the toroid, the size of the exhaust profile was similar to the control. Analysis of the different time steps for the axial test also revealed 
that as time increased the magnetic field had a more significant effect on the exhaust profile. This is clearly visible when comparing Figure 51, Figure 52, and Figure 53 in Appendix G.

\section{Orientation Comparisons at Each Time Step}

This section compares data from each orientation at a single time step. At the 10 second time step, the two tangential tests were nearly identical in shape and only differed slightly total size. The toroid test had a similar shape as the tangential tests but had the smallest overall exhaust profile based on Table 6. The axial test produced the largest overall exhaust profile with a significantly different shape than the other profiles at the 10 second time step.

In the 20 second time step the toroid test and the tangential south test, in Figure 43 and Figure 49 respectively, both started and ended larger than the control test, but were smaller than the control immediately in front of and behind the toroid. In this time step the tangential north test profile was consistently less than or equal to the control test profile, while the axial test was consistently larger than the control. Interestingly, data from Table 6 indicates the total plume reduction in the toroid and tangential north tests were very similar even though the tangential north test did not follow the same profile trend as the toroid or tangential south tests at that time step. It is also worth noting that the total change in plume size for the toroid and tangential tests was lower in the 20 second time step than both the 10 and 30 second time steps. This phenomenon can clearly be seen in Table 6.

During the 30 second time step each configuration had a slightly different exhaust profile. In this time step the tangential north test profile was uniformly smaller than the control test. While the toroid test expanded toward the end of the flow field Table 6 revealed its total profile reduction was almost identical to the tangential north test. The tangential south test began at a similar size to the control test, became smaller than the control immediately in front of and behind the toroid, then expanded larger than the control test toward the end of the flow field. Finally, the axial test profile was uniformly larger than all other tests including the control test.

\section{Comparison with Toroid Test}

This section describes a comparative analysis between the toroid test and each apparatus configuration containing a magnetic field. Descriptions of each exhaust profile were determined using a combination of the trends indicated in Table 7 and false color images displayed in Appendix H. Table 7 presents the percent change in area between the toroid test and each magnetic 
field configuration. In Table 7 positive numbers indicate the exhaust profile was larger than the toroidal test and negative numbers indicate the profile was smaller than the toroidal test.

Table 7: Exhaust plume percent expansion trends in comparison to the toroid test

\begin{tabular}{|c|c|c|c|}
\hline & 10 seconds & 20 seconds & 30 seconds \\
\hline Toroid vs. tangential north [\%] & +1.01 & -0.12 & +0.04 \\
\hline Toroid vs. tangential south [\%] & +1.30 & +0.53 & +0.50 \\
\hline Toroid vs. axial (north or south) [\%] & +3.29 & +3.44 & +4.57 \\
\hline Toroid STD [\%] & +1.42 & +1.52 & +1.99 \\
\hline
\end{tabular}

The first data set compared with the toroid test was the tangential north data. The bottom of the tangential north plume was larger at all time steps in comparison to the toroid test plume. However, the top of the plume is only consistently larger for all time steps immediately before and after the application of a magnetic field for each time step. For the 20 and 30 second time steps the beginning and end of the flow field are smaller for the tangential north exhaust plume. This resulted in the tangential north test having a very small increase in the exhaust profile during the 30 second time step and a decrease in the overall exhaust profile during the 20 second time step. This indicates that the plasma was being attracted by the magnetic field but that the field was not strong enough to have a lasting effect on the exhaust profile. See Figure 54, Figure 55, and Figure 56 in Appendix $\mathrm{H}$ for a visual comparison of each time step.

Very little change could be observed for the comparison between the tangential south test and the toroid test. At 10 seconds, both the top and bottom edges of the exhaust profile were larger for the tangential south plume. At 20 seconds the tangential south plume was only larger at the bottom edge and at 30 seconds the tangential south plume was only larger at the top edge. While the tangential south exhaust profile for all time steps was larger than the toroid test profile, data from Table 7 indicates that the effectiveness of the tangential south magnetic field decreased as time progressed. See Figure 57, Figure 58, and Figure 59 in Appendix H for a visual comparison of each time step.

For all time steps the axial test produced a significantly larger exhaust profile than the toroid test. Additionally, comparing each time step yielded a transient result where the exhaust plume expanded wider as time progressed. See Figure 60, Figure 61, and Figure 62 in Appendix $\mathrm{H}$ for a visual comparison of each time step. 


\section{Conclusion}

After thorough analysis of the experimental findings described in the Results and Discussion sections of this thesis several observations were made. First, data from Table 6 and Table 7 as well as photographic evidence displayed in the appendices clearly show that manipulating the exhaust profile of a hypersonic plasma jet is possible under near vacuum conditions. Comparisons with the control test and the toroid test revealed that the axial configurations provided the most significant exhaust profile expansion across all time steps. Without accounting for the flow trajectory alteration that skewed data displayed in Table 6 the axial test plume will provide the largest overall exhaust profile within another 10 seconds of operation. This claim operates under the assumption that the axial test exhaust profile will continue its expansion trend beyond the 30 second time parameter in this experiment.

For applications where constricting the exhaust flow is most desirable the numerical analysis from Table 6 and Table 7 revealed that a toroidal structure alone, without the influence of a magnetic field, would produce the best results when considering the entire exhaust plume. However, Figure 55 and Figure 56 from Appendix $\mathrm{H}$ indicate that tangential north configuration does decrease the plume size after passing through the toroid. This further supports the claim that permanent magnets mounted on a steel toroid will effectively alter the exhaust profile through magnetoplasmadynamics.

The secondary objective in this investigation was to determine if a series of permanent magnets mounted on a steel toroid could serve as a practical replacement for toroidal electromagnets. For experimentation purposes in this project permanent magnets were lower cost and more versatile in their ability to produce a variety of magnetic field configurations. Electromagnets can be more versatile in their ability to produce a variety of field strengths, however, that was not the subject of this study.

A numerical analysis revealed that, for practical applications on satellites or space probes, permanent magnets provide a more feasible solution for long-term use. While electromagnets can produce much stronger magnetic field the added weight of a power supply large enough to support an electromagnet of equivalent size and strength to the toroidal structure in this study would be impractical due to the strict weight requirements used to design spacecraft.

In conclusion, this study was successful in demonstrating that a toroidal magnetic field can manipulate the exhaust profile of a plasma jet was achieved under near vacuum conditions using 
only permanent magnets mounted on a steel toroid. Based on calculations performed using Equation 6 and Equation 7 the study also revealed that using permanent magnets is more practical than using a toroidal electromagnet with the same magnetic field strength for long term use. This is because permanent magnets do not require an external power source and would continue to work if the temperature of the toroidal structure does not exceed the maximum operating temperature of the magnets. 


\section{References}

[1] J. Anderson, Fundamentals of Aerodynamics, 5th ed., New York: McGraw-Hill, 2011.

[2] T. Colonius, S. Lee and P. Moin, "The Free Compressible Viscous Vortex," Journal of Fluid Mechanics, vol. 230, no. 1, pp. 45-73, 1991.

[3] J. Sellers, W. Astore, R. Giften and W. Larson, Understanding Space: An Introduction to Astronautics, 3rd ed., D. Kirkpatrick, Ed., New York: McGraw-Hill Higher Education, 2005.

[4] J. Sellers, W. Astore, R. Giften and W. Larson, "Rockets and Launch Vehicles," Understanding Space: An Introduction to Astronautics, 3rd ed., New York, McGraw-Hill Higher Education, 2005, pp. 533-607.

[5] F. Ebersohn, S. Girimaji, D. Staack, J. Shebalin, B. Longmier and C. Olsen, "Magnetgic Nozzle Plasma Plume: Review of Crucial Physical Phenomena," 48th AIAA/ASME/SAE/ASEE Joint Propulsion Conference \& Exhibit, Atlanta, Georgia, 2012.

[6] K\&J Magnetics, Inc., "Neodymium Magnet Physical Properties," [Online]. Available: https://www.kjmagnetics.com. [Accessed 23 November 2016].

[7] APW Company, "Electromagnets \& Custom Coils," [Online]. Available: http://apwelectromagnets.com. [Accessed 20 March 2017].

[8] E. Choueiri, "A Critical History of Electric Propulsion: The First 50 Years (1906-1956)," Journal of Propulsion and Power, vol. 20, no. 4, pp. 193-203, 2004.

[9] M. Martinez-Sanchez and J. Pollard, "Spacecraft Electric Propulsion - An Overview," Journal of Propulsion and Power, vol. 14, no. 5, pp. 688-699, 1998.

[10] H. Tobari, A. Ando, M. Inutake and K. Hattori, "Characteristics of Electromagnetically Accelerated Plasma Flow in an Externally Applied Magnetic Field," Physics of Plasmas, vol. 14, no. 9, p. 093507, 2007.

[11] M. Inutake, A. Ando, F. Hori, T. Sugimura, K. Fukushi, T. Ochiai, M. Yamamoto, T. Yagai, A. Imasaki and M. Yoshinuma, "Supersonic Plasma Flow in a Magnetic Nozzle," Japan Society for Aeronautical and Space Sciences, Aoba, Sendai, 1999.

[12] M. Coletti, "A Thrust Formula for an MPD Thruster with Applied-Magnetic Field," Acta Astronautica, vol. 81, no. 2, pp. 667-674, 2012.

[13] T. Boyd and J. Sanderson, The physics of plasmas, Cambridge: Cambridge University Press, 2007. 
[14] R. Nave, "HyperPhysics," Georgia State University, 2001. [Online]. Available: http://hyperphysics.phy-astr.gsu.edu/hphys.html. [Accessed 11 June 2017].

[15] L. Graham, "Feasibility Study of Enthalpy, Density, and Velocity Probe for Low Density Supersonic Flows," 1973.

[16] A. Drews, L. Cademartiri, G. Whitesides and K. Bishop, "Electric Winds Driven by Time Oscillating Corona Discharges," Journal of Applied Physics, vol. 114, no. 14, p. 143302 , 2013.

[17] S. Utyuzhnikov, A. Konyukhov, D. Rudenko, S. Vasil'evskii, A. Kolesnikov and O. Chazot, "Simulation of Subsonic and Supersonic Flows in Inductive Plasmatrons," AIAA Journal, vol. 42, no. 9, pp. 1871-1877, 2004.

[18] Ace Hardware, "Apex 9.5" 1501b. Capacity Shopping Cart Wheel (SC9013-P03)," [Online]. Available: http://www.acehardware.com/product/index.jsp?productId=18209376. [Accessed 23 Nobember 2016].

[19] J. Matthey, "Neodymium, Nd," MatWeb - The Online Materials Information Resource, [Online]. Available: http://www.matweb.com/search/datasheet. [Accessed 8 December 2016].

[20] C. Goldbach and G. Nollez, "Thermal Arc Plasmas as Radiation Sources," Astronomy and Astrophysics, vol. 21, no. 2, pp. 299-322, 1972.

[21] Y. Cengel and A. Ghajar, Heat and Mass Transfer: Fundamentals \& Applications, 5th ed., New York, NY: McGraw-Hill Education, 2015.

[22] K. Fournier, M. Cohen, M. May and W. Goldstein, "Ionization State Distribution and Radiative Cooling Rate for Argon in a Low-Density Plasma," Atomic Data and Nuclear Data Tables, vol. 70, no. 2, pp. 231-254, 1998.

[23] Brooks Instrument, LLC, "Industrial Glass Tube, Variable Area Flowmeters," 2014.

[24] AutoZone, "Odyssey Battery," [Online]. Available: http://www.autozone.com/batteriesstarting-and-charging/battery/odyssey-battery/832116_702844_0. [Accessed 4 April 2017]. 


\section{Appendix A: MATLAB Code Calculating Magnetic Field Strength}

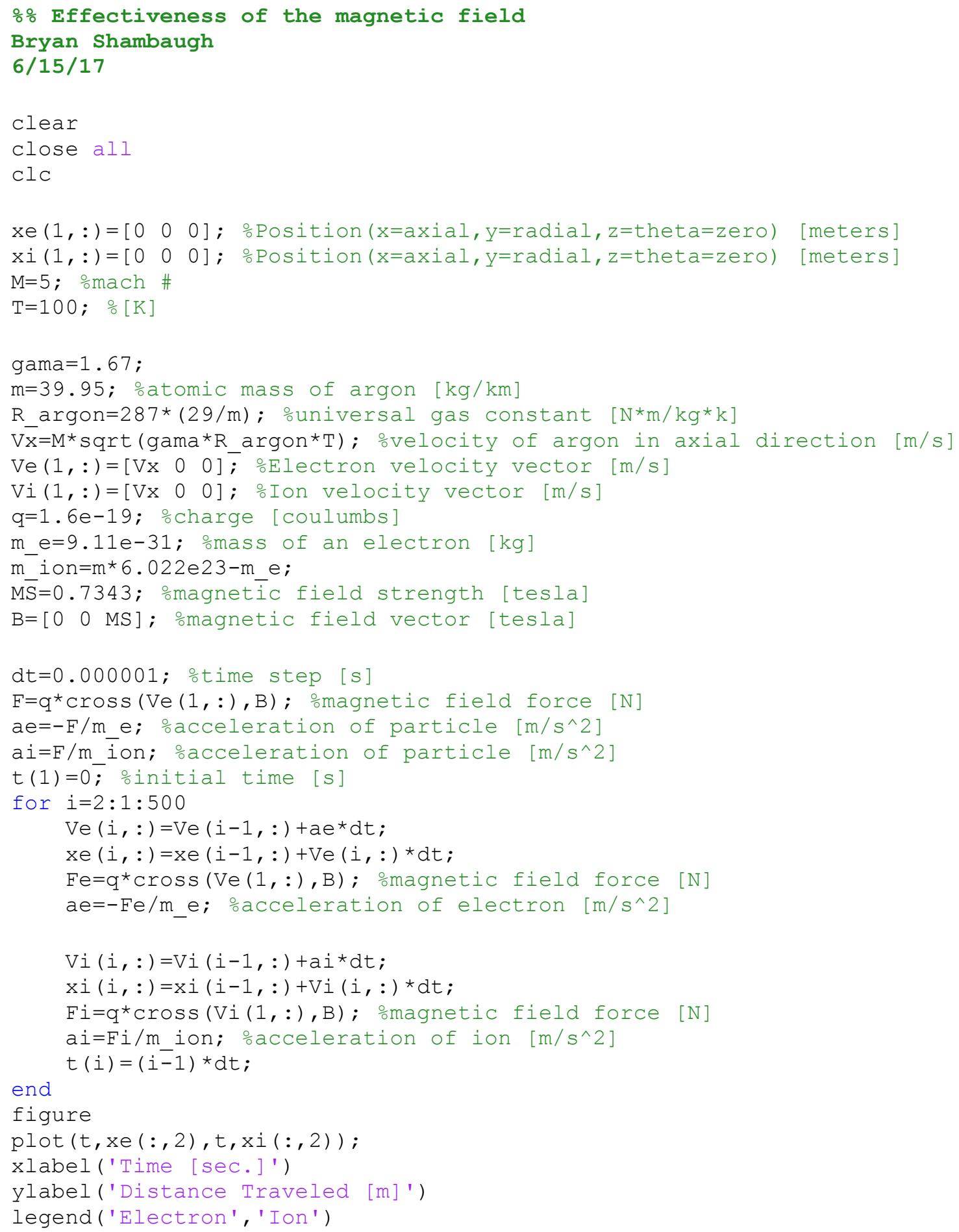




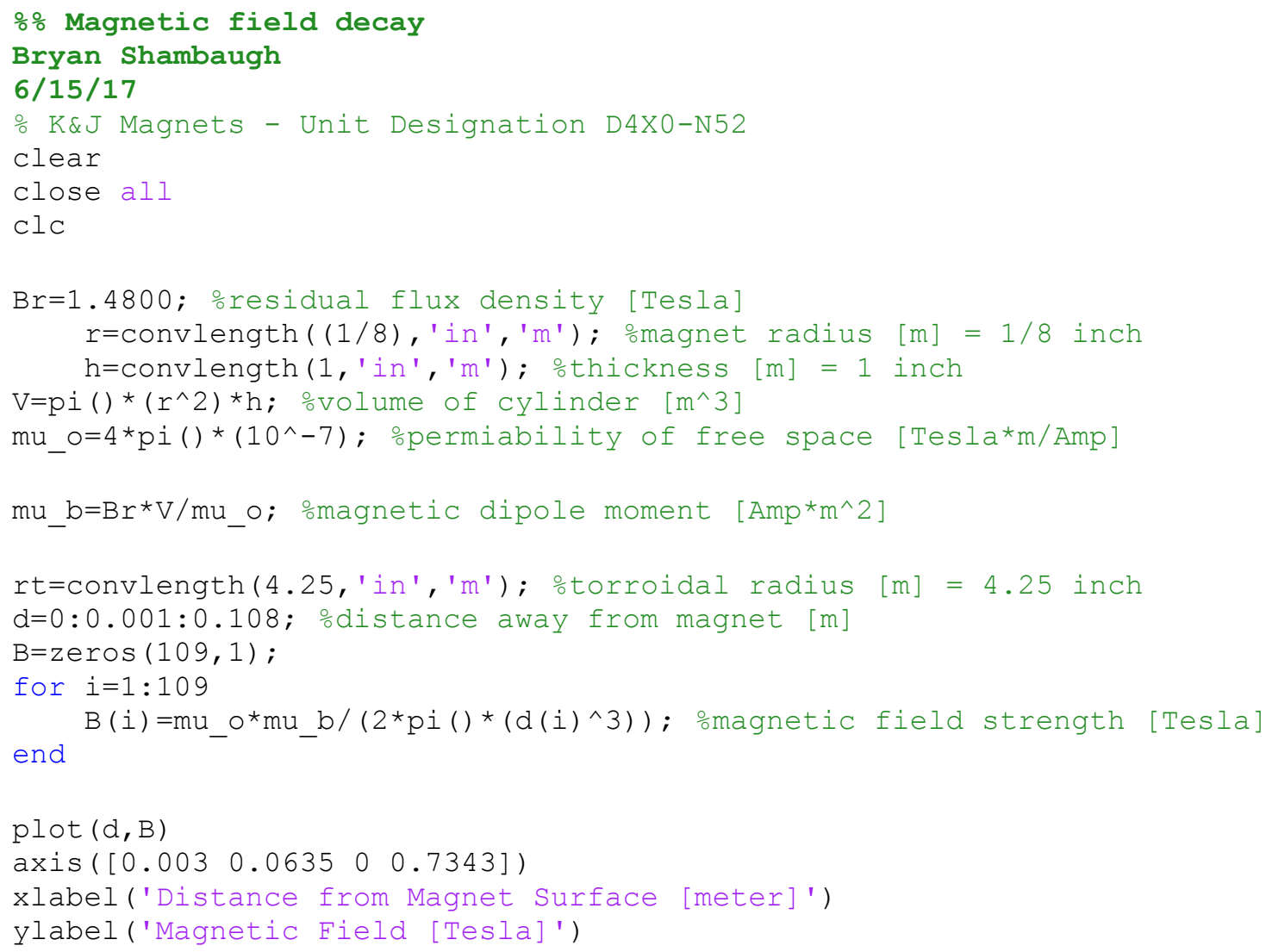




\section{Appendix B: Data Set 1}

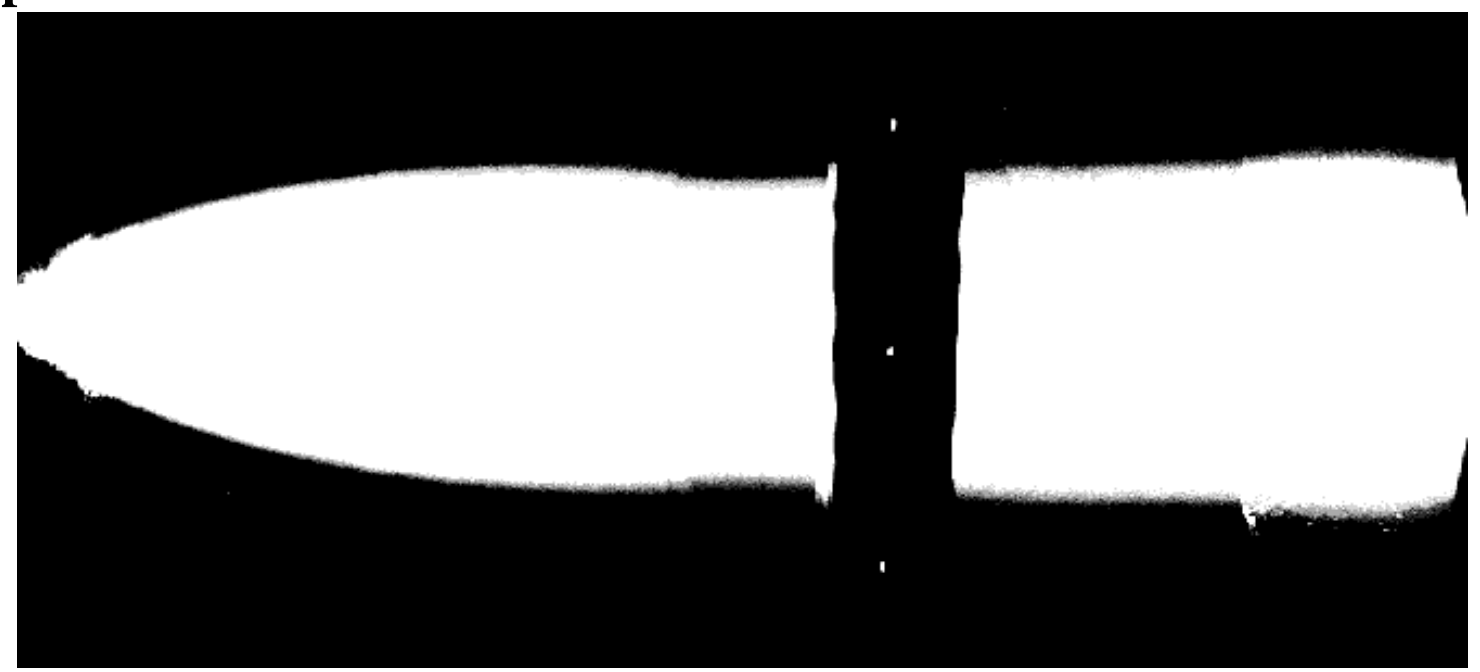

Figure 33: Toroid test (10 sec.)

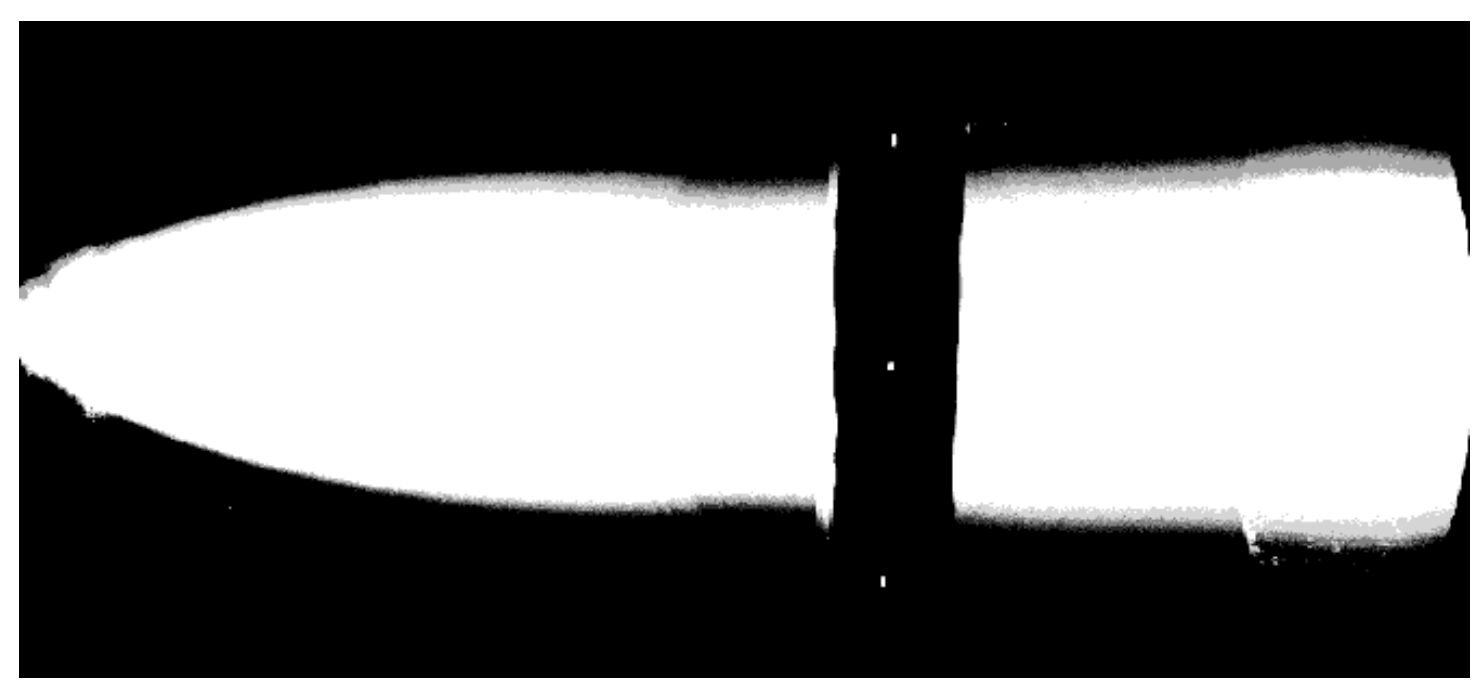

Figure 34: Toroid test (20 sec.)

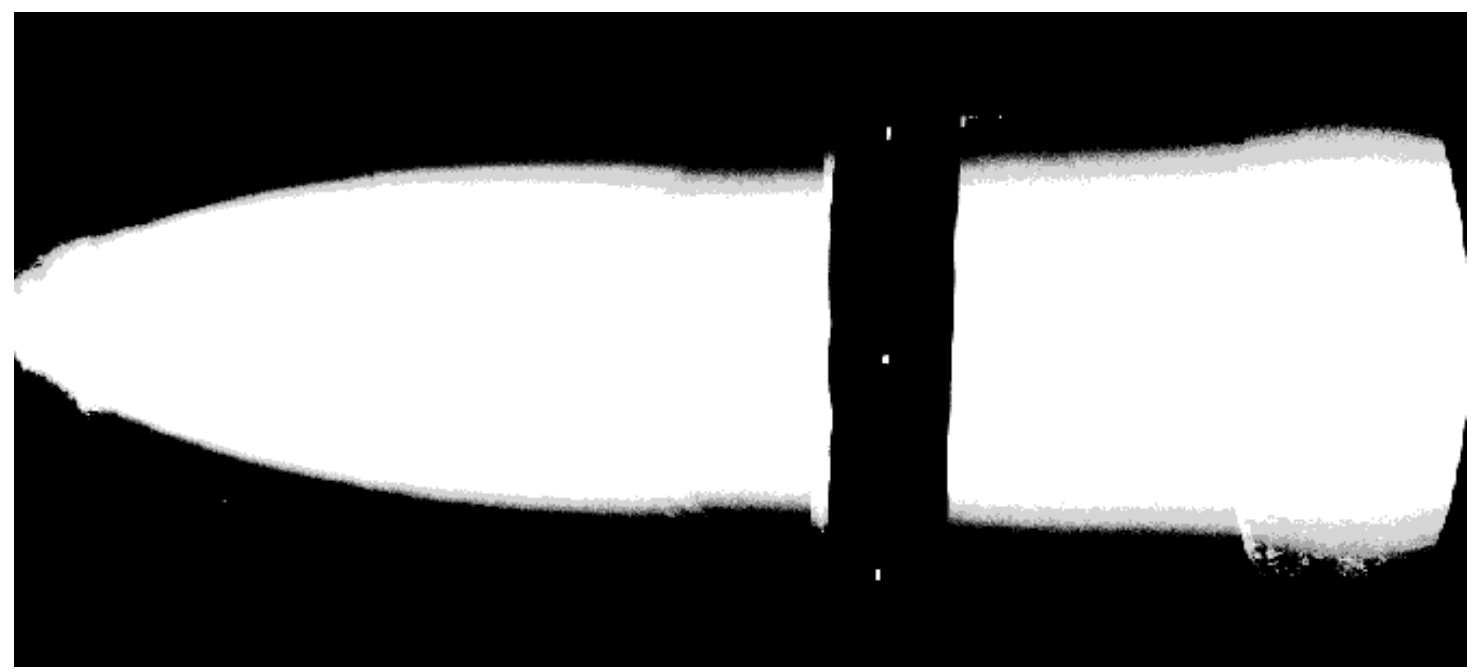

Figure 35: Toroid test (30 sec.) 


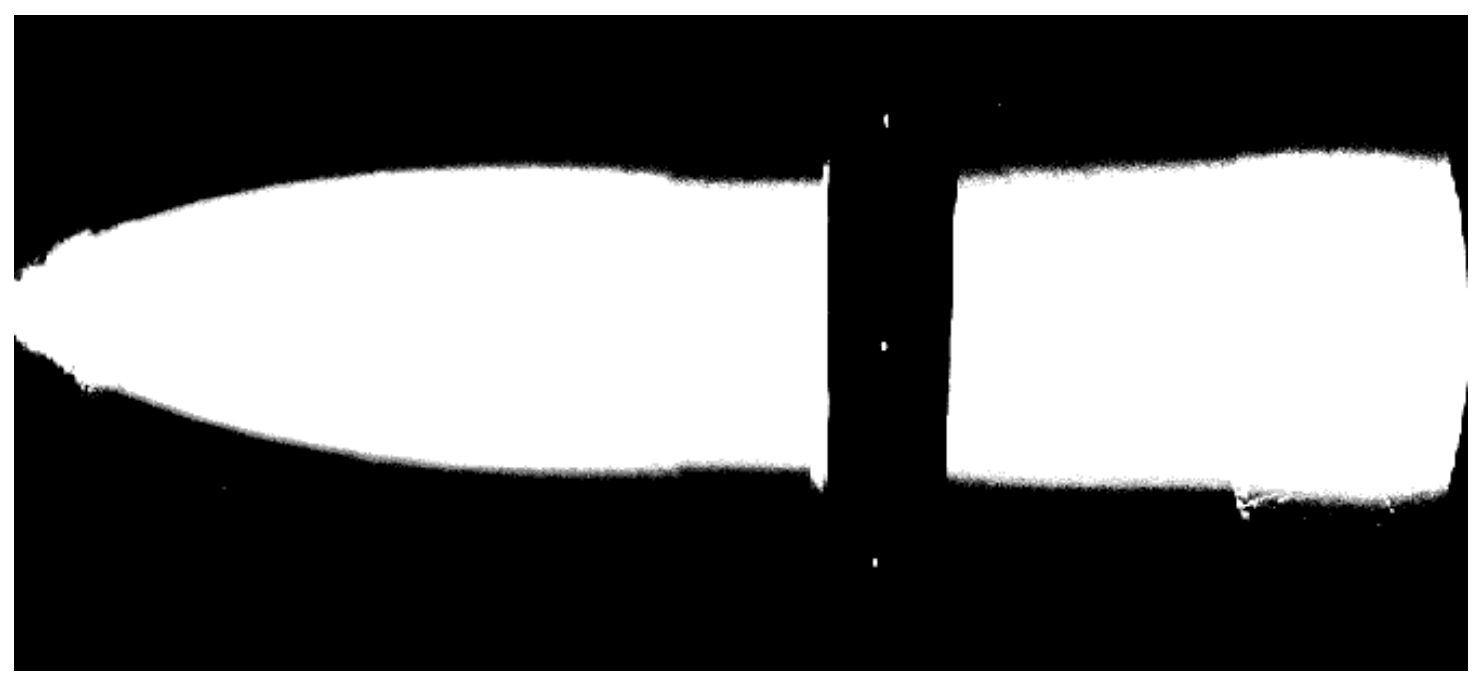

Figure 36: Axial north test (10 sec.)

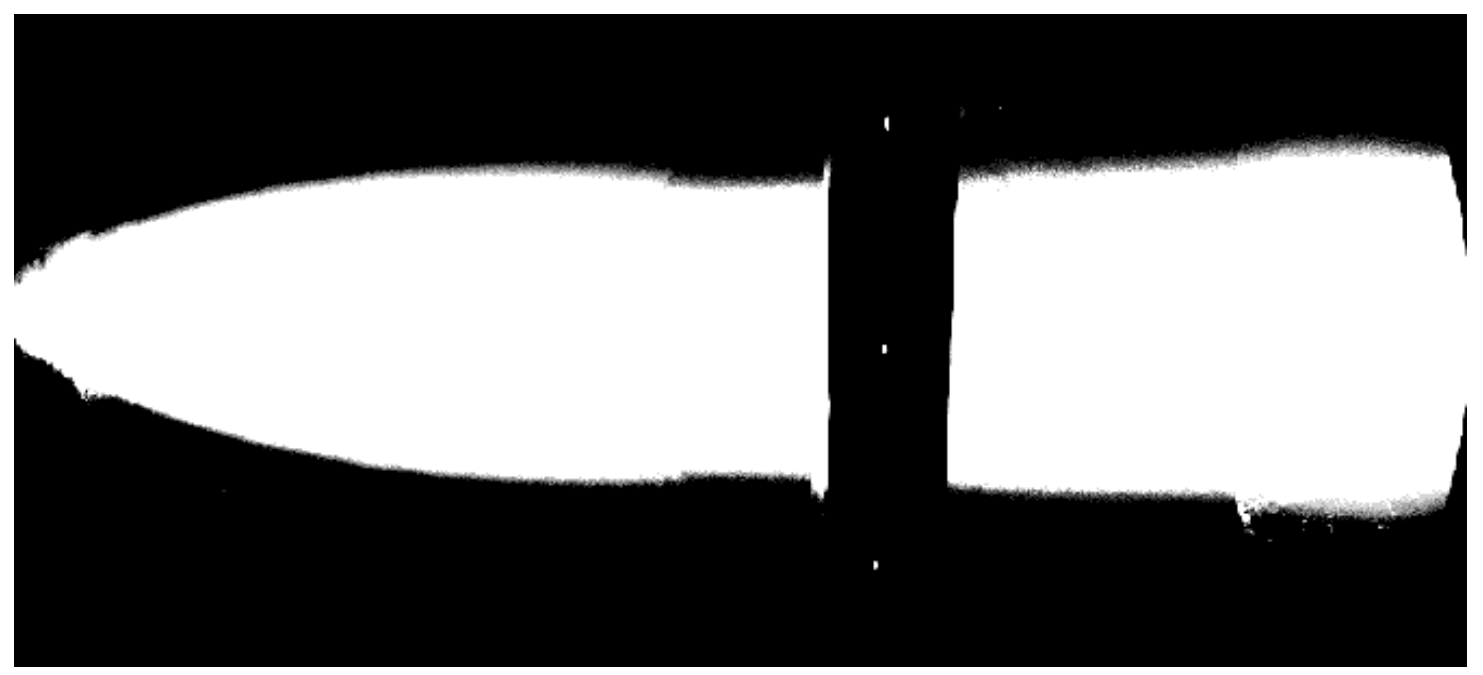

Figure 37: Axial north test (20 sec.)

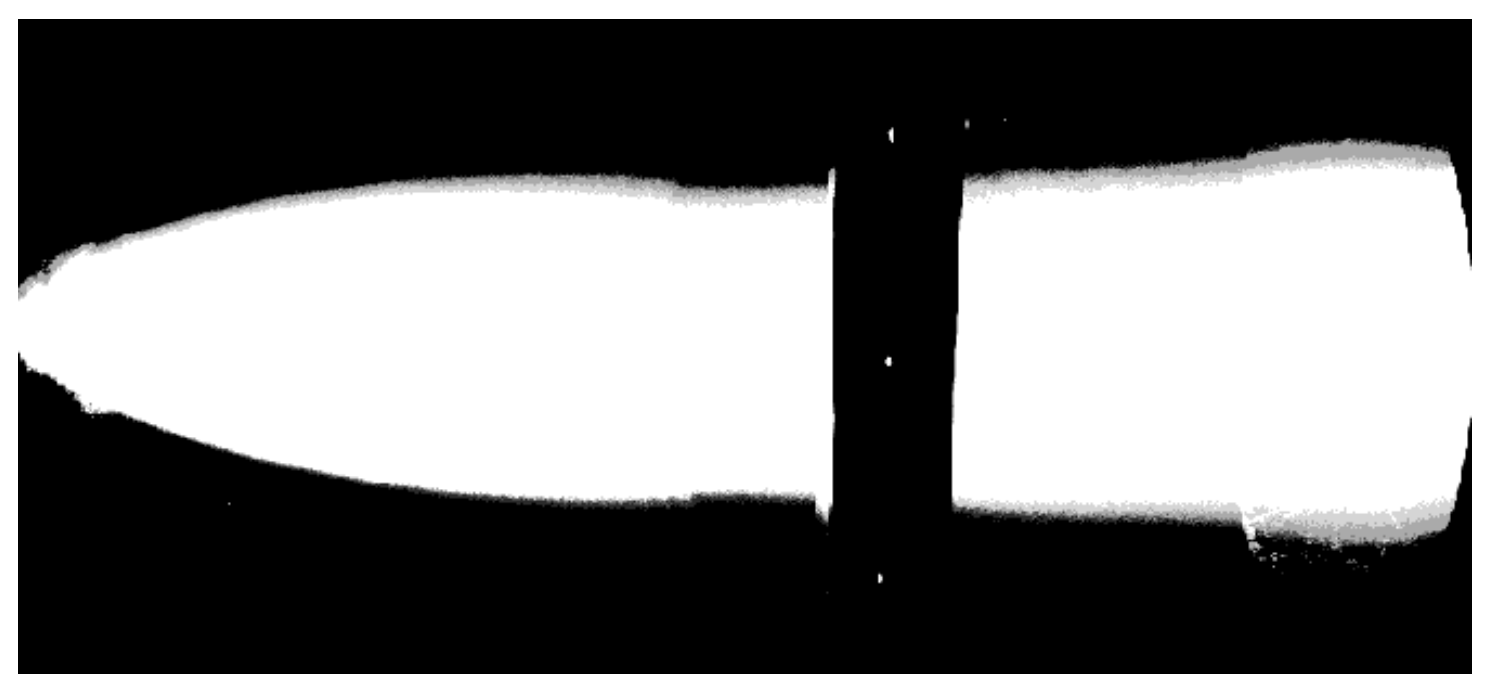

Figure 38: Axial north test (30 sec.) 


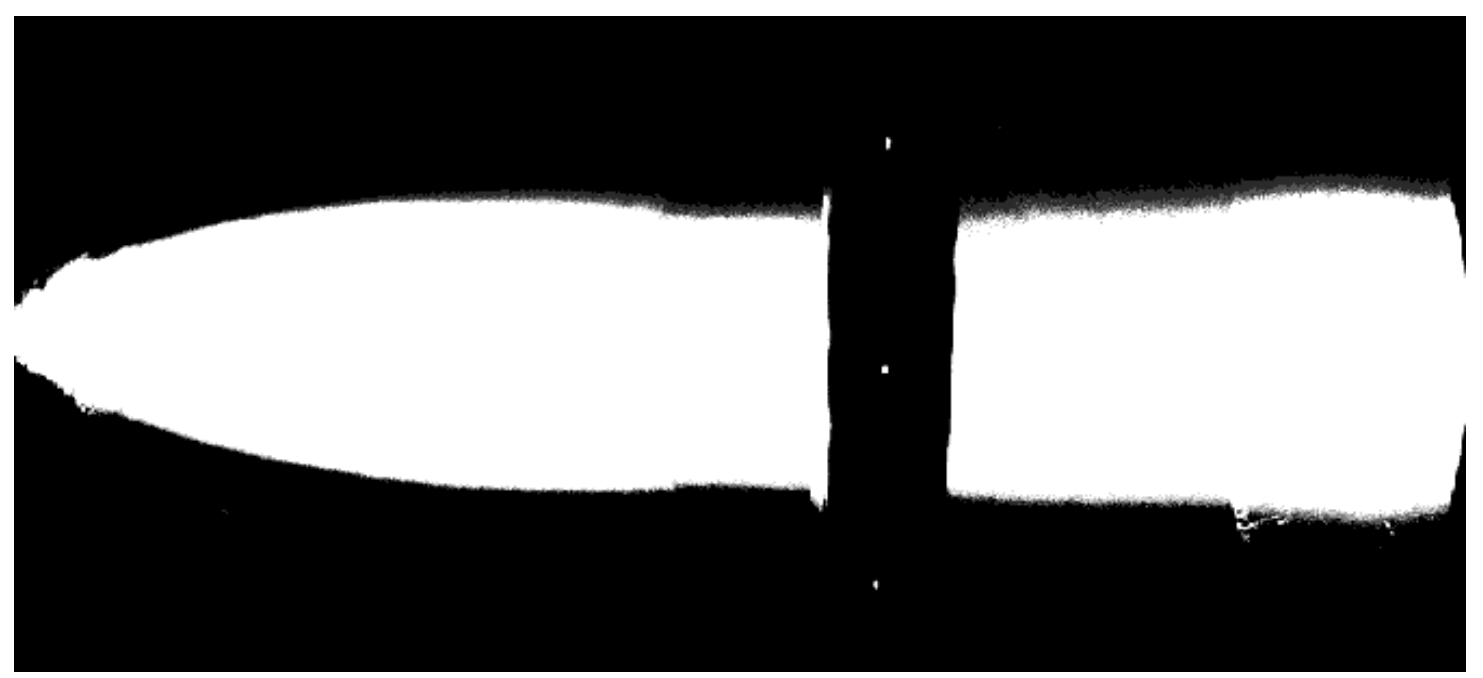

Figure 39: Axial south test (10 sec.)

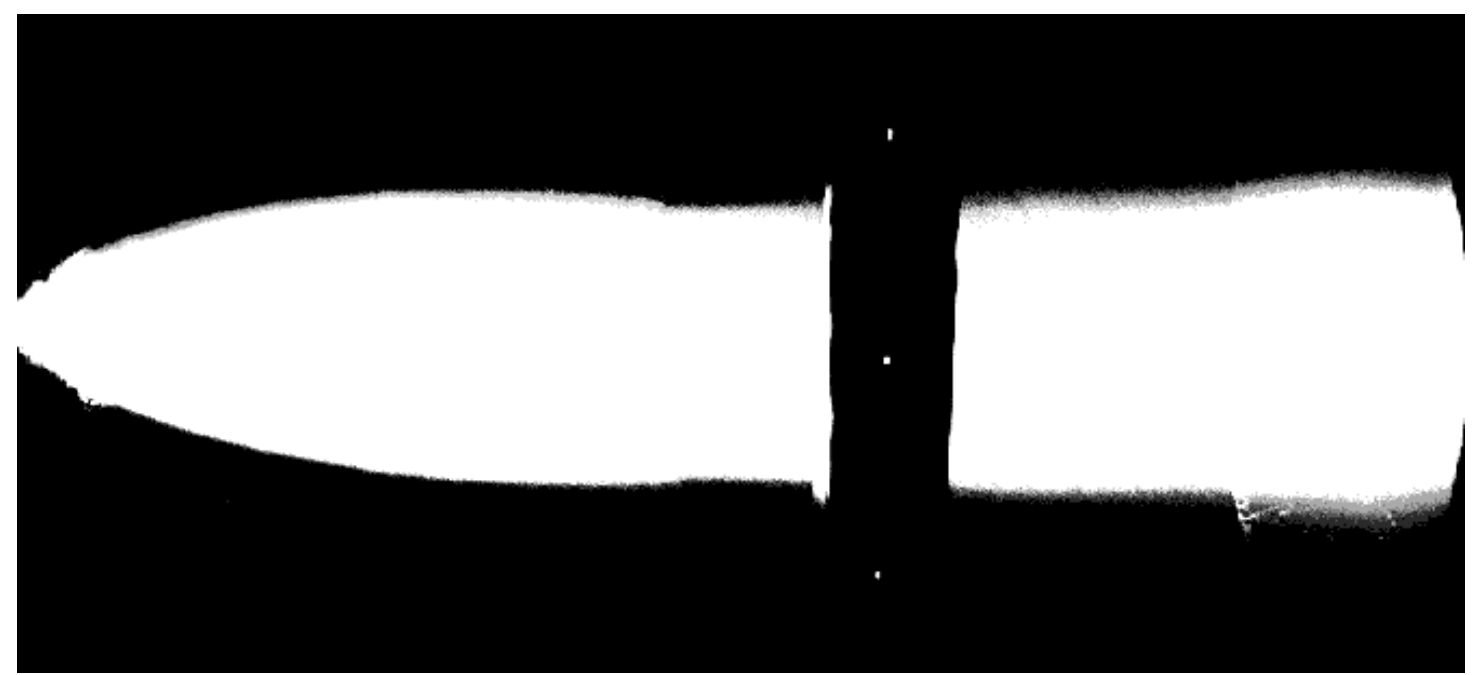

Figure 40: Axial south test (20 sec.)

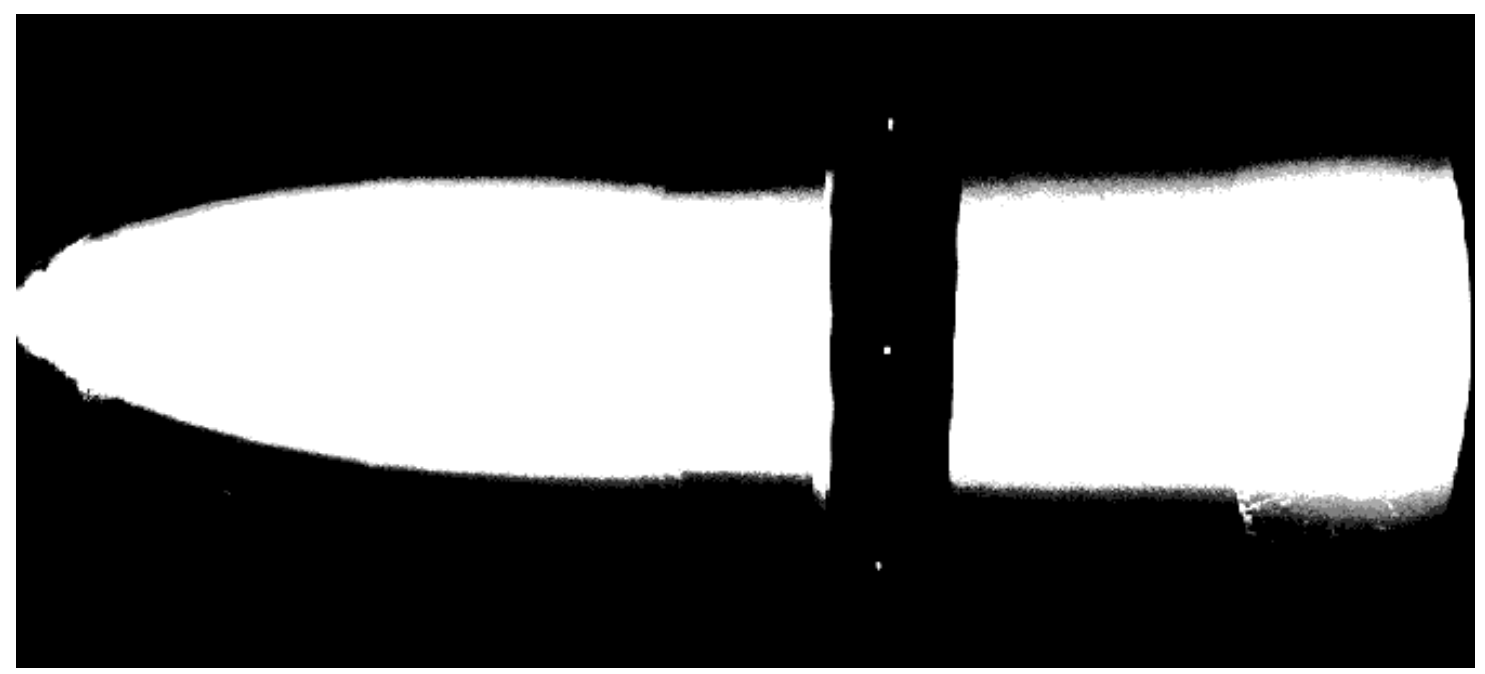

Figure 41: Axial south test (30 sec.) 


\section{Appendix C: MATLAB Code for Data Set One}

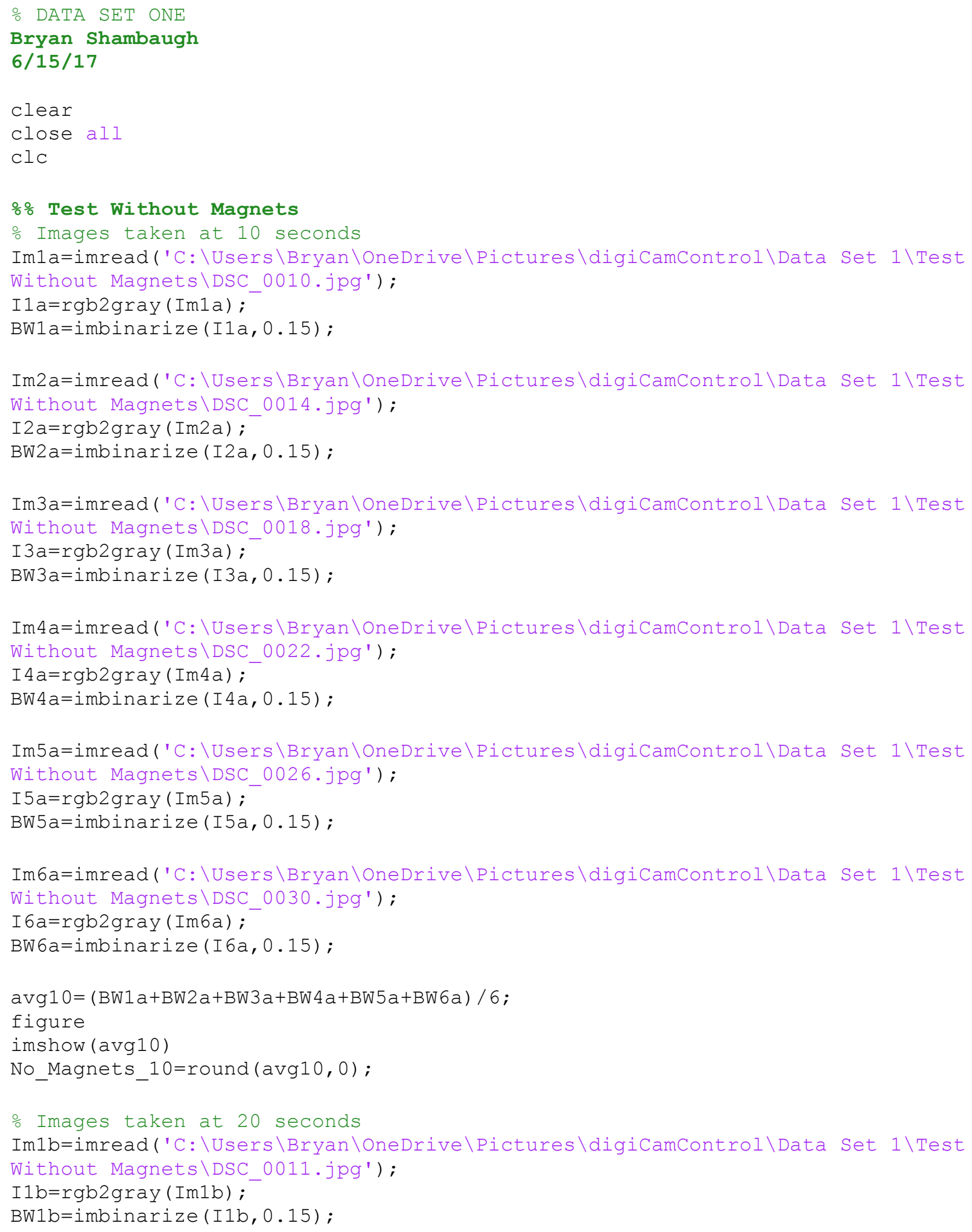




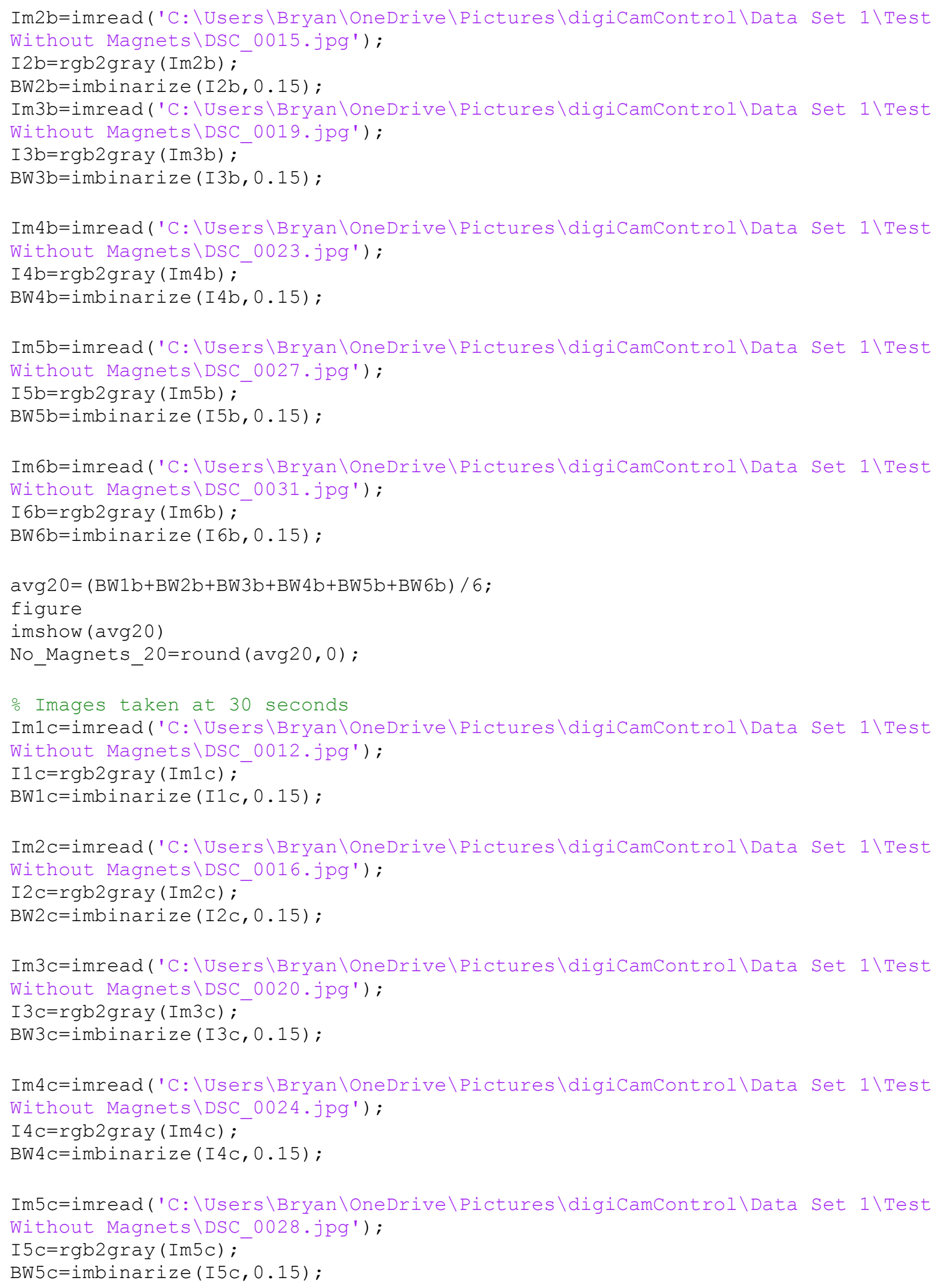




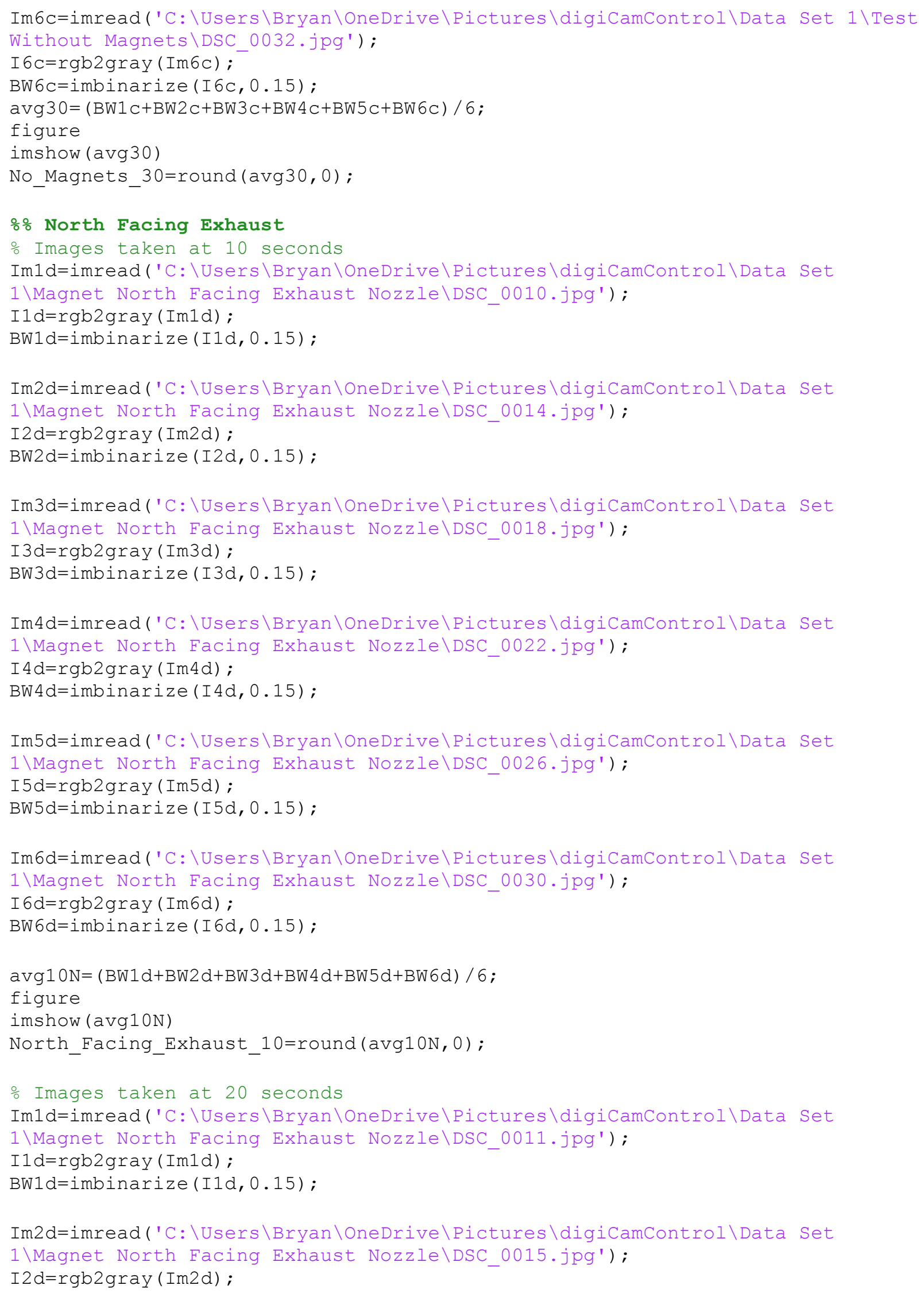




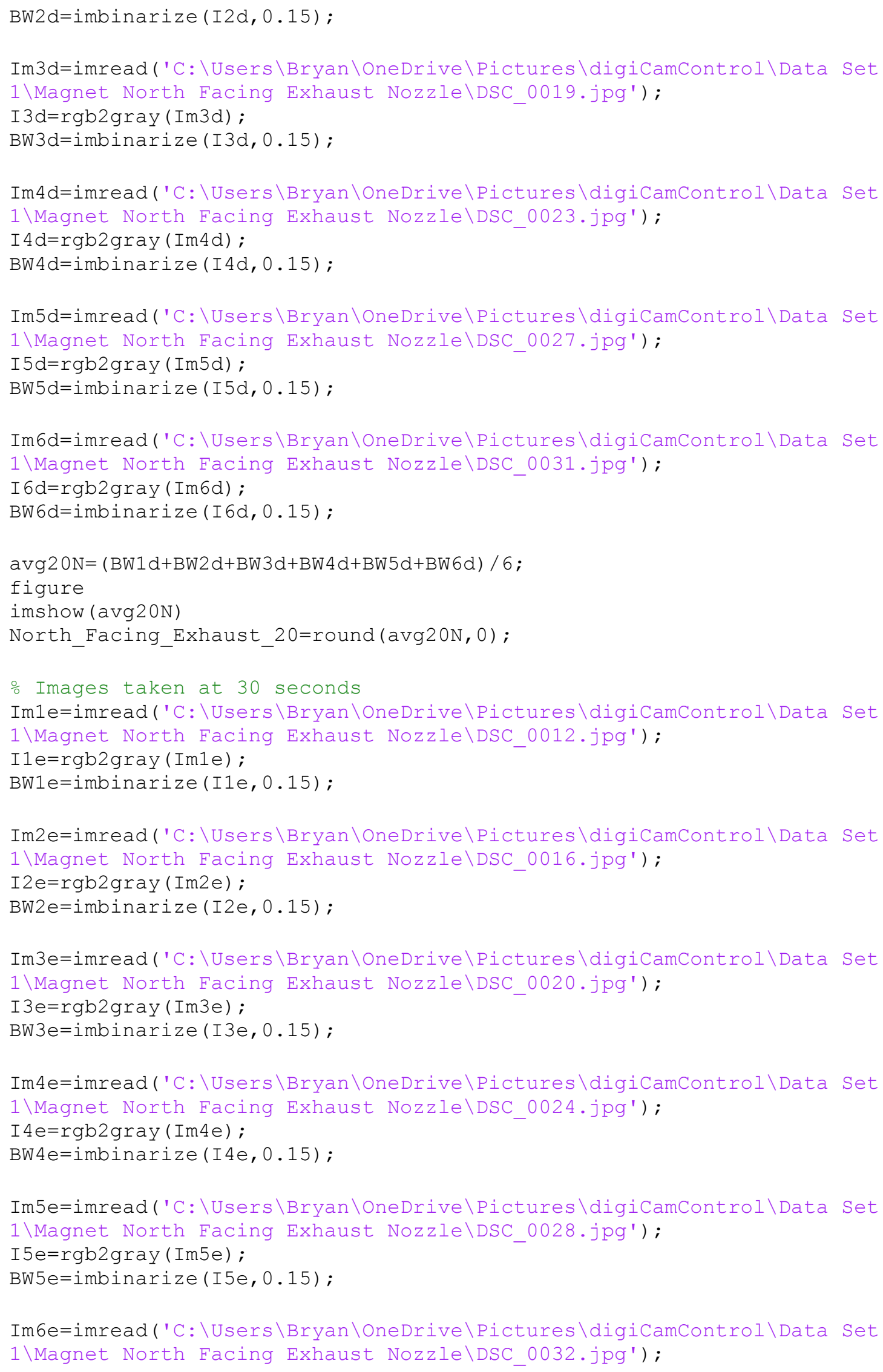




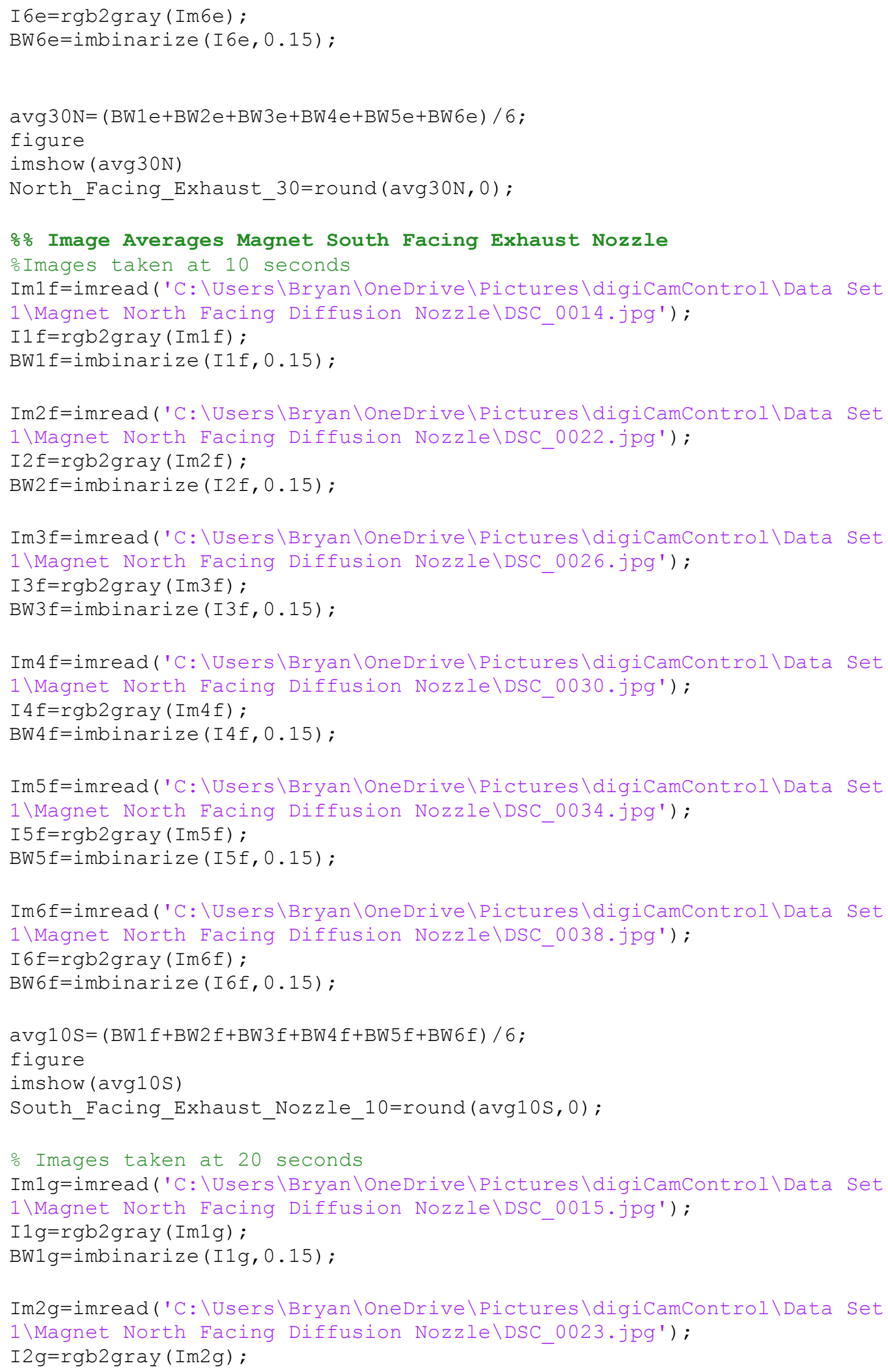




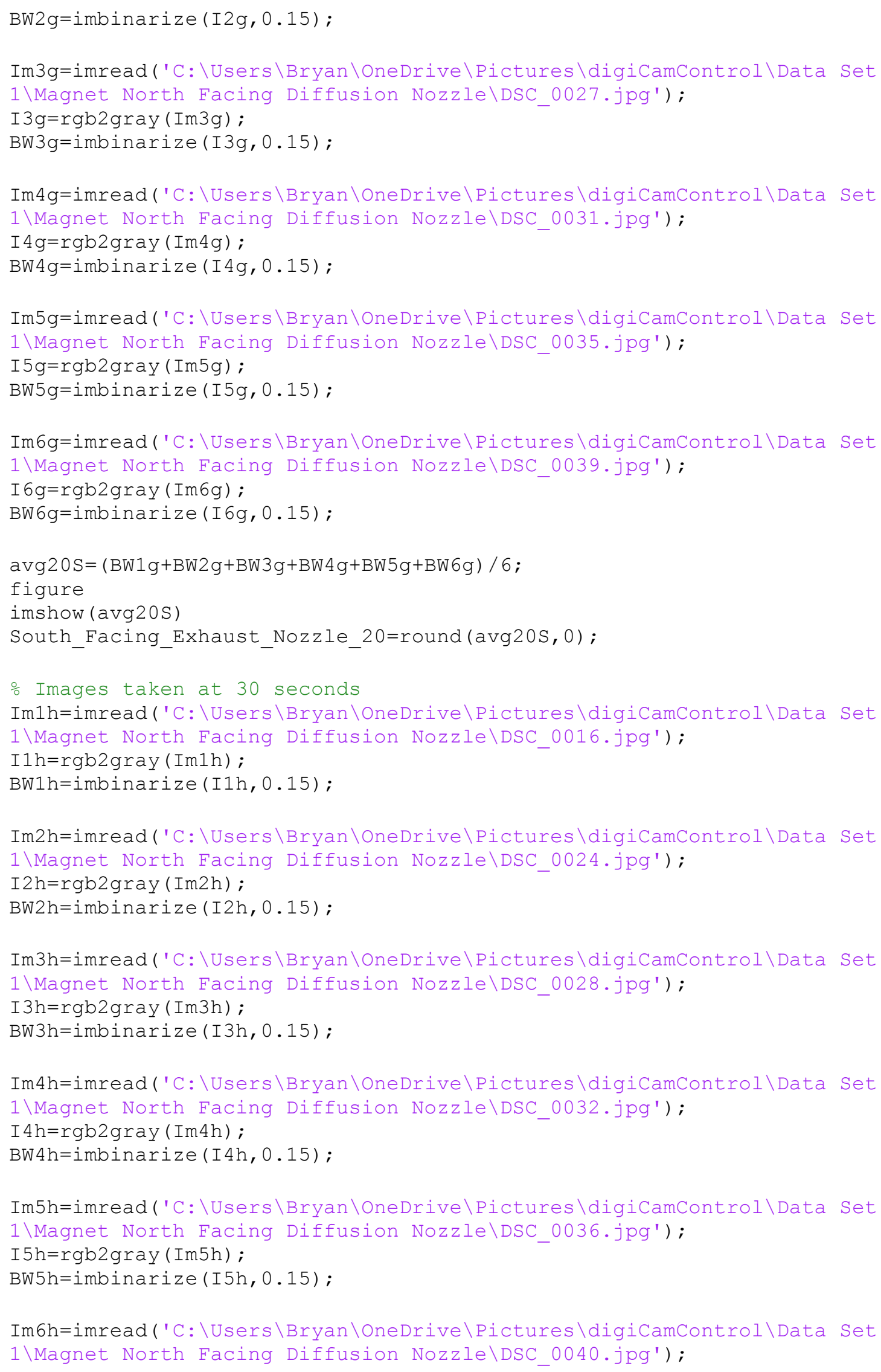




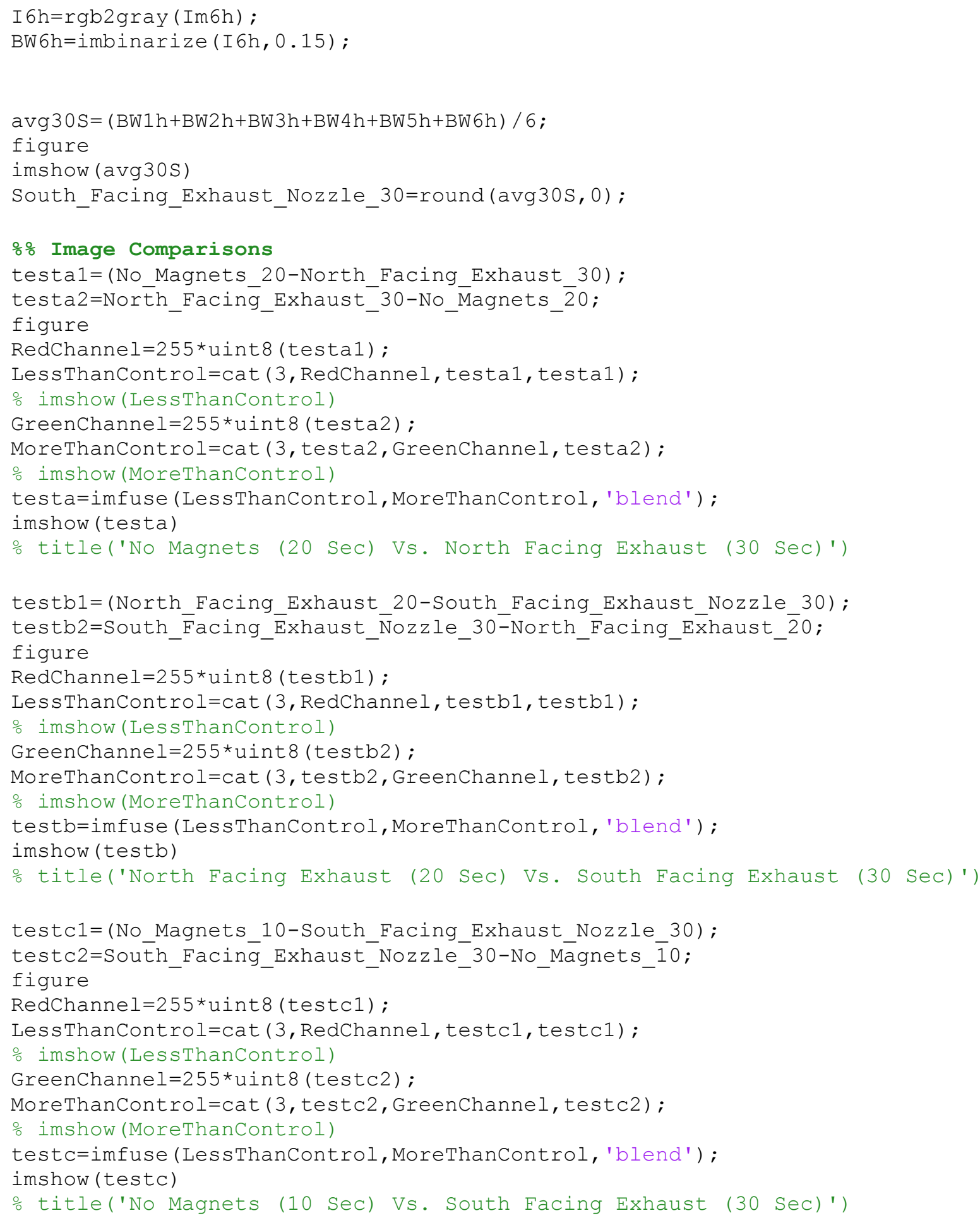




\section{Appendix D: MATLAB Code for Data Set 2 at 10 seconds}

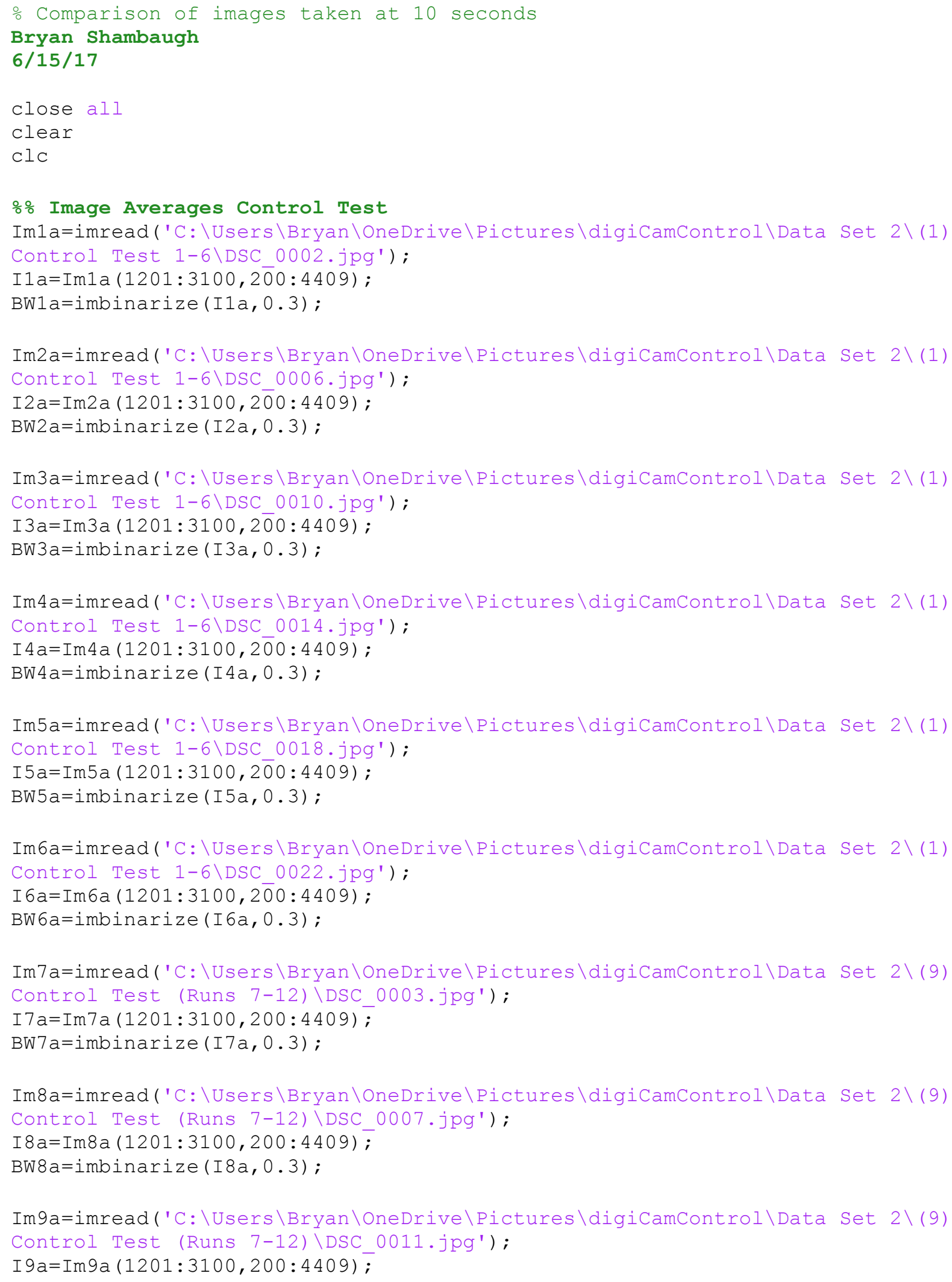




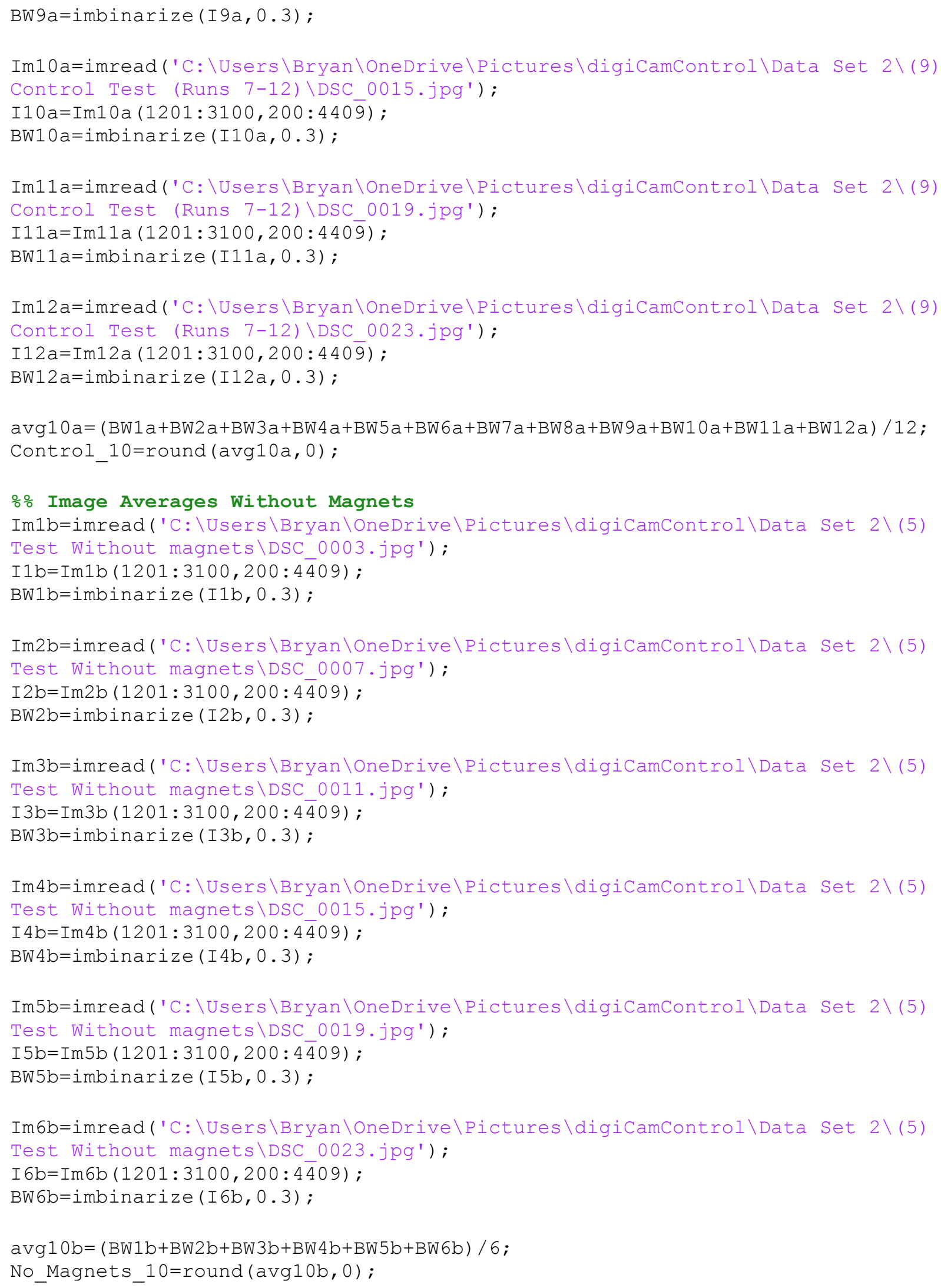




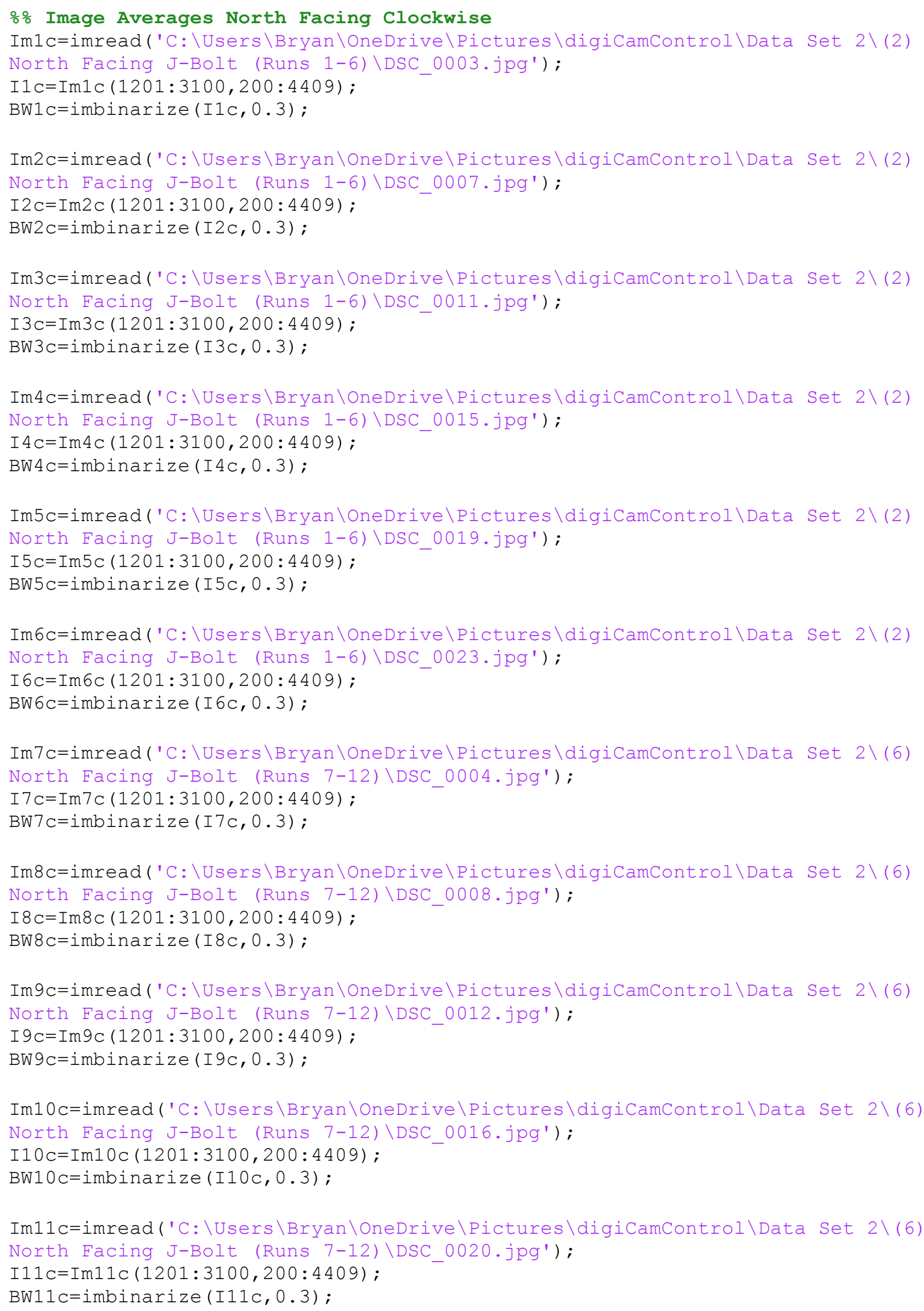




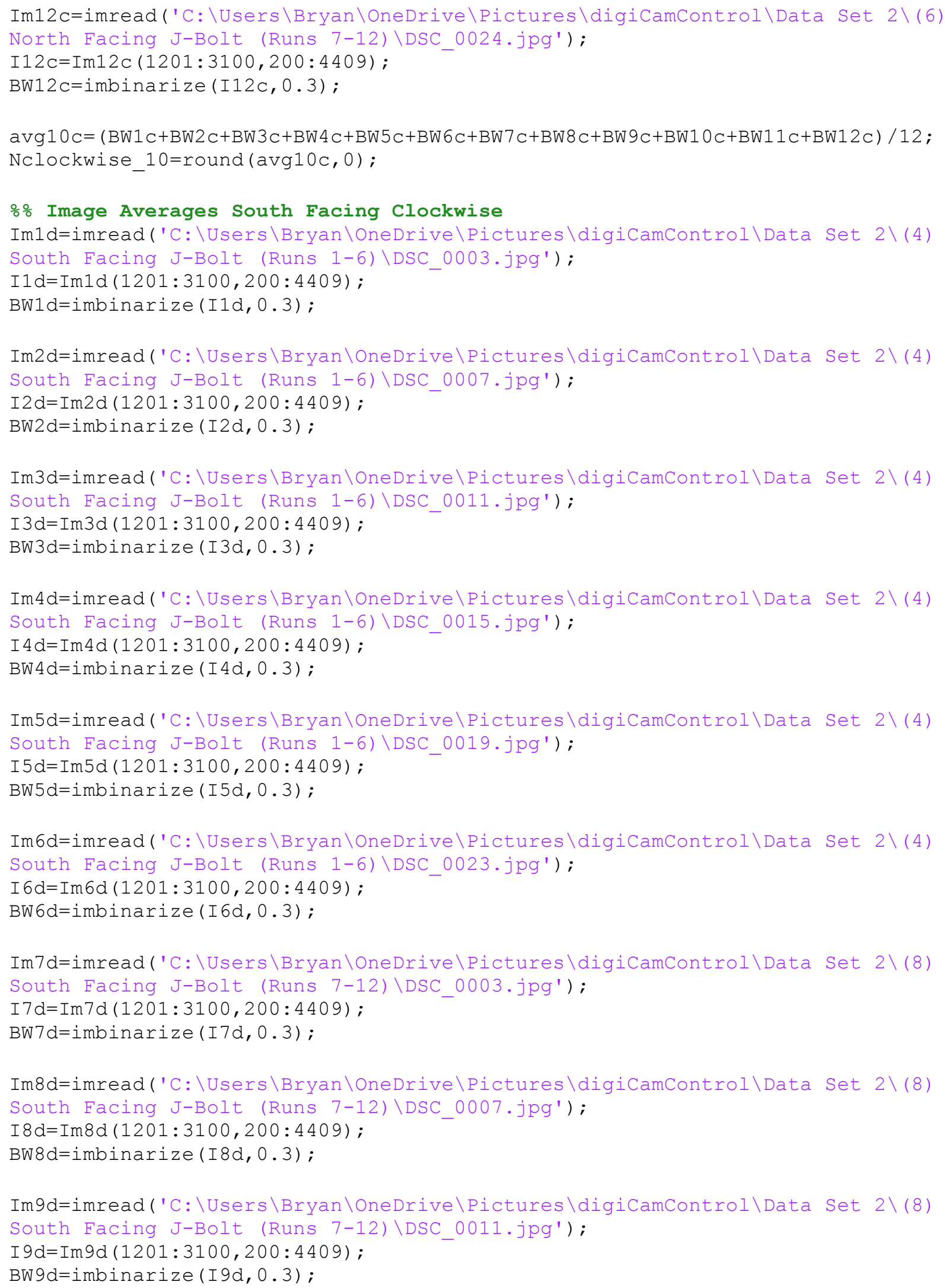




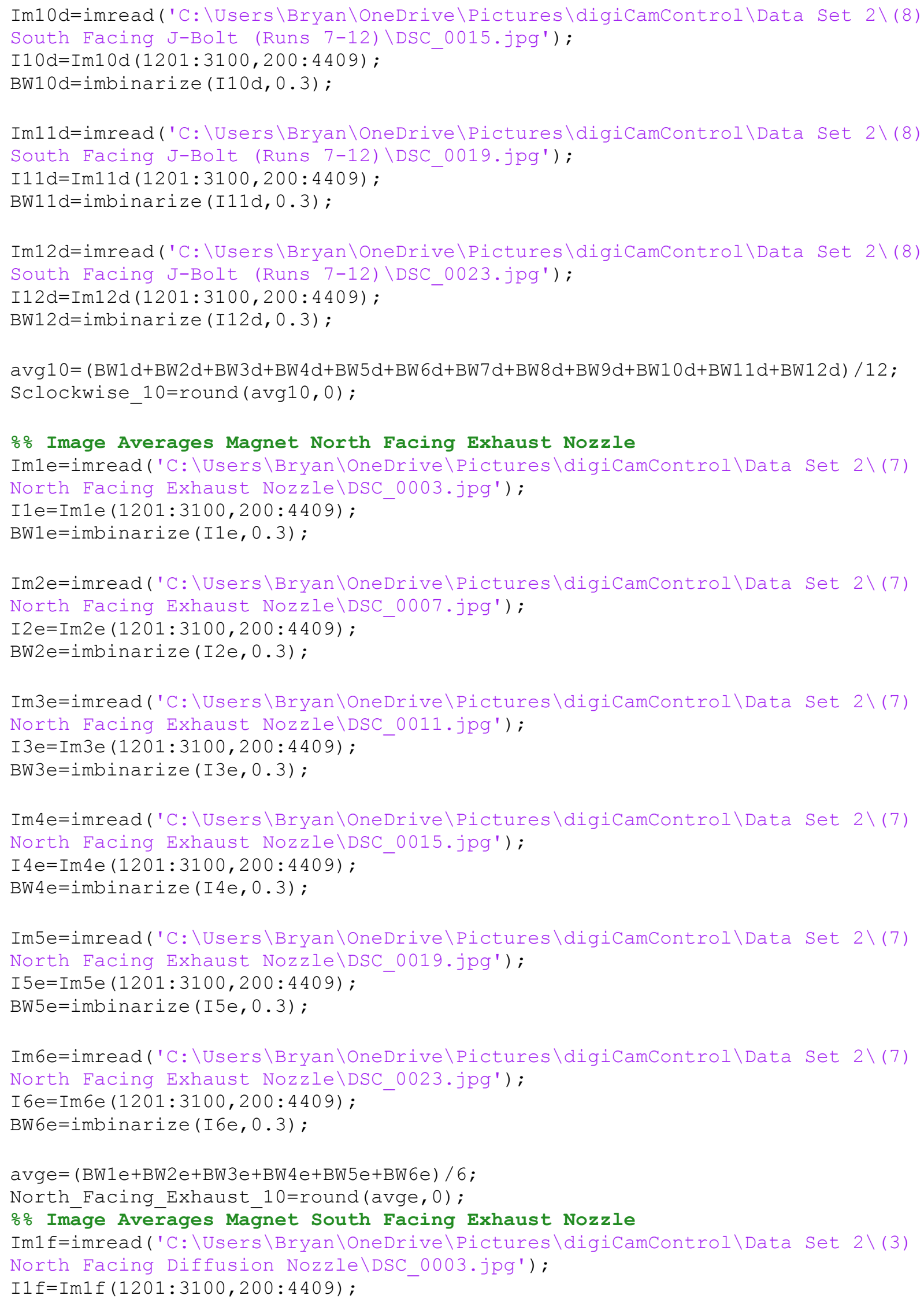




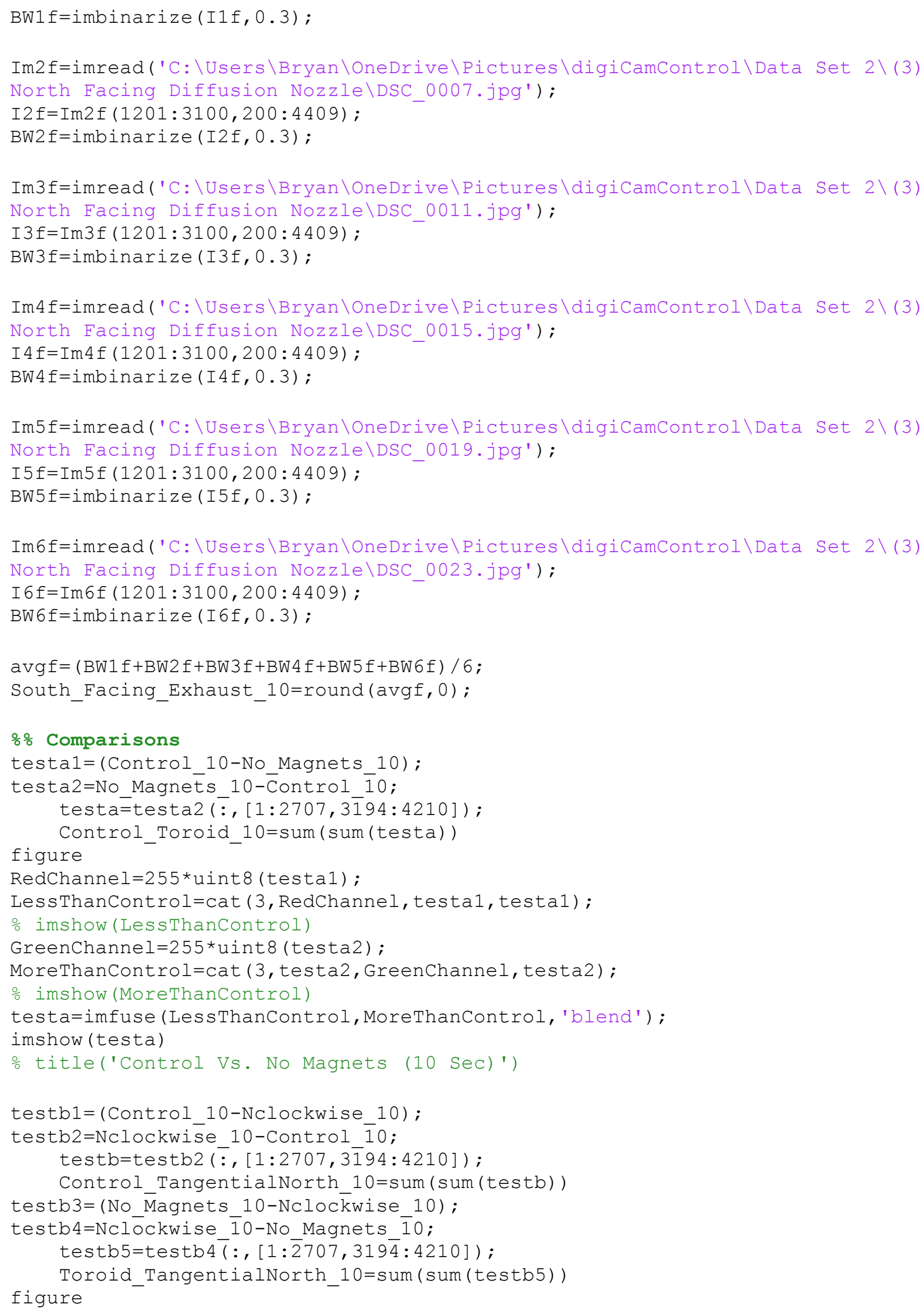




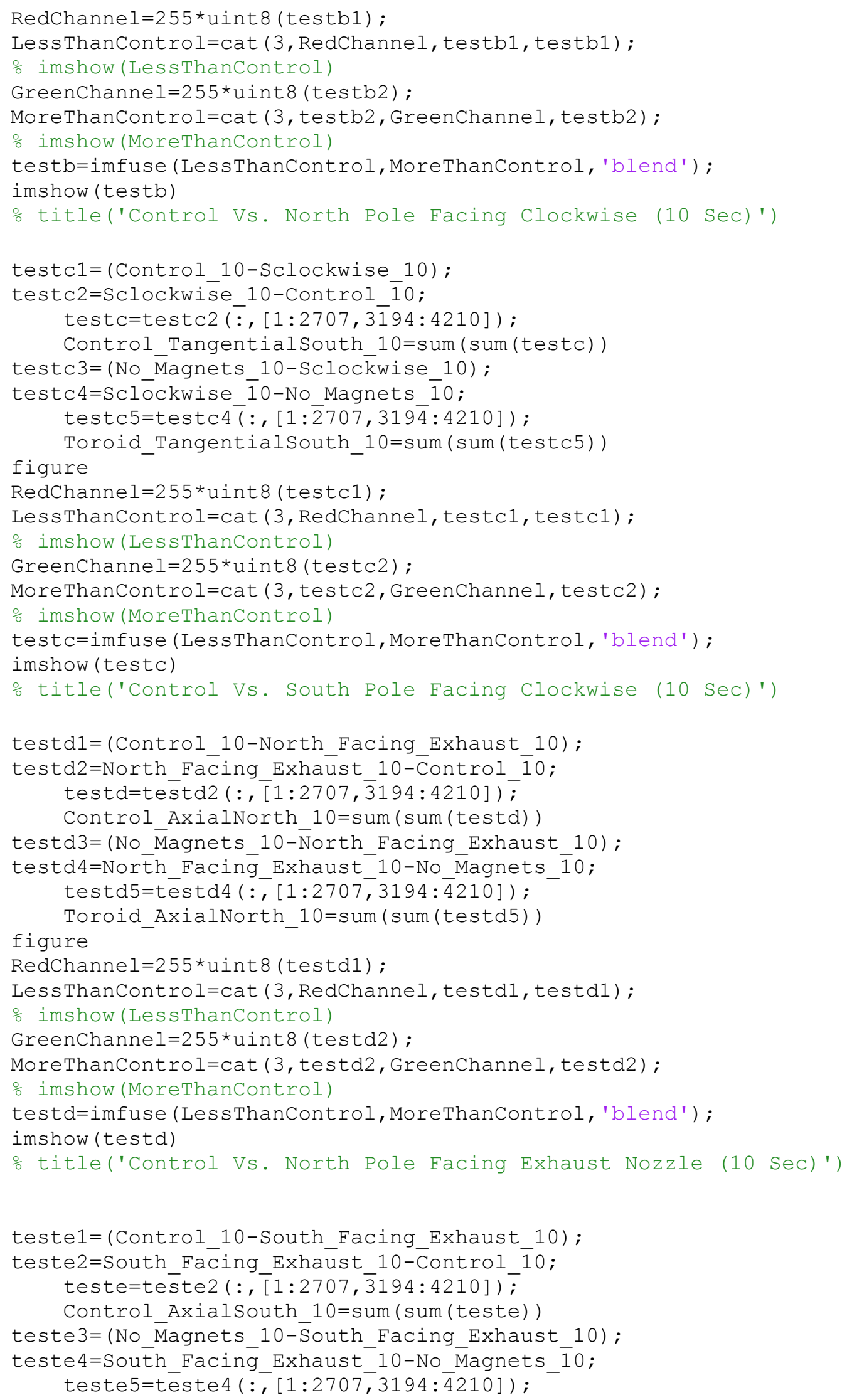


figure

Toroid_AxialSouth_10=sum (sum(teste5))

RedChannel $=255 *$ uint8 (teste1);

LessThanControl=cat (3, RedChannel, teste1, teste1) ;

\% imshow (LessThanControl)

GreenChannel $=255$ *uint 8 (teste2);

MoreThanControl=cat (3, teste2, GreenChannel, teste2) ;

\% imshow (MoreThanControl)

teste=imfuse (Less ThanControl, MoreThanControl, 'blend' ) ;

imshow (teste)

\% title('Control Vs. South Pole Facing Exhaust Nozzle (10 Sec)') 


\section{Appendix E: MATLAB Code for Data Set 2 at 20 seconds}

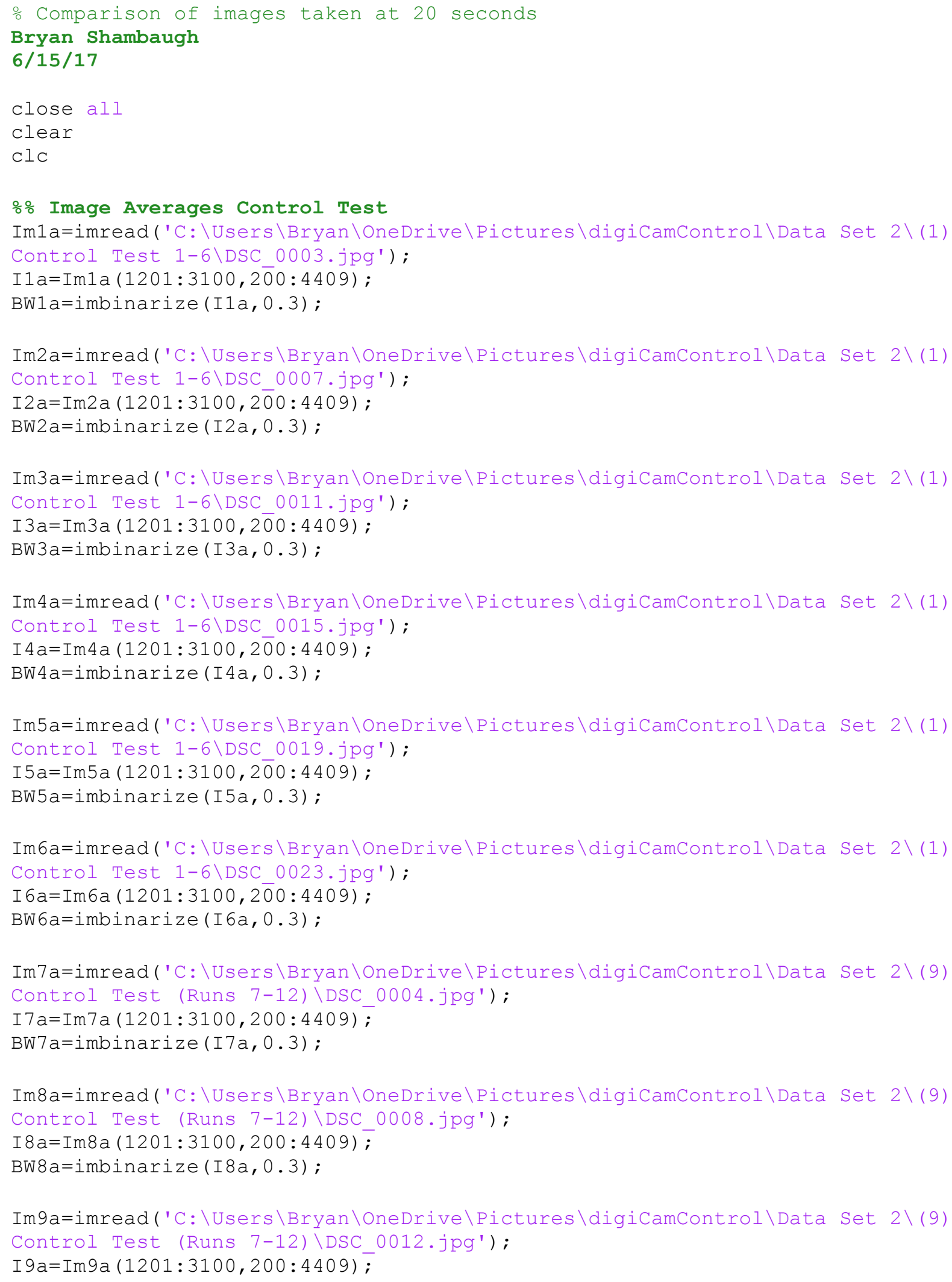




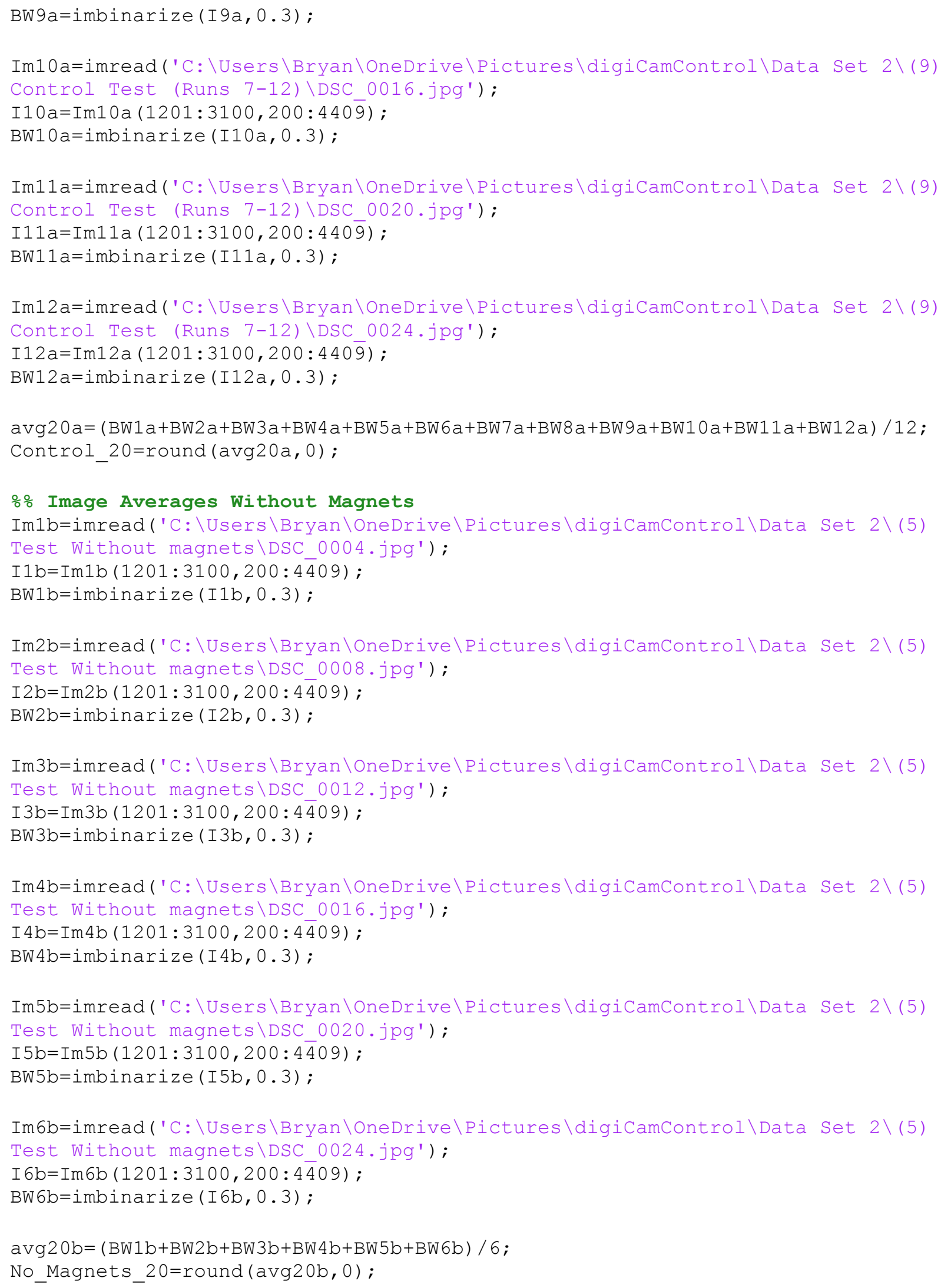




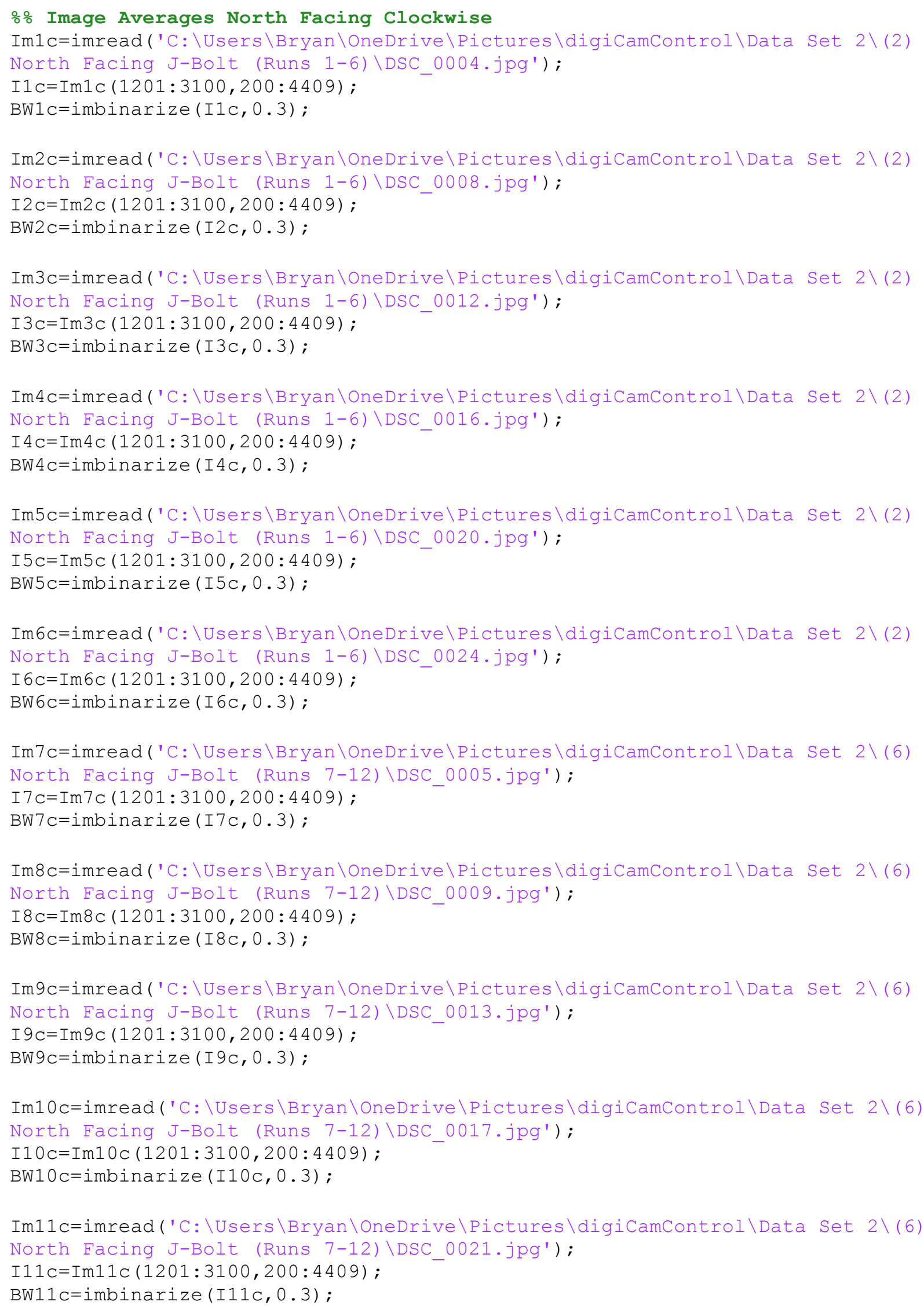




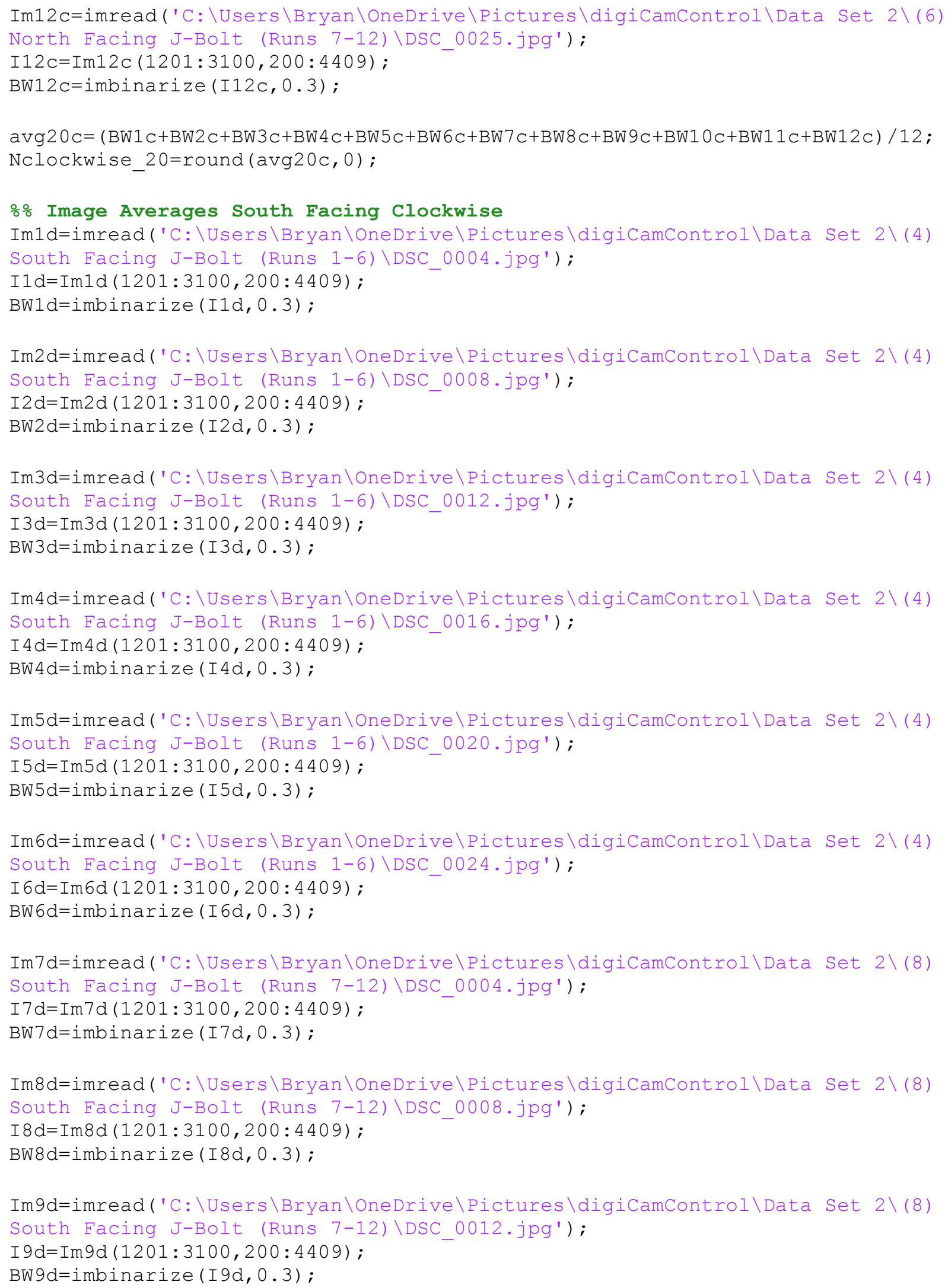




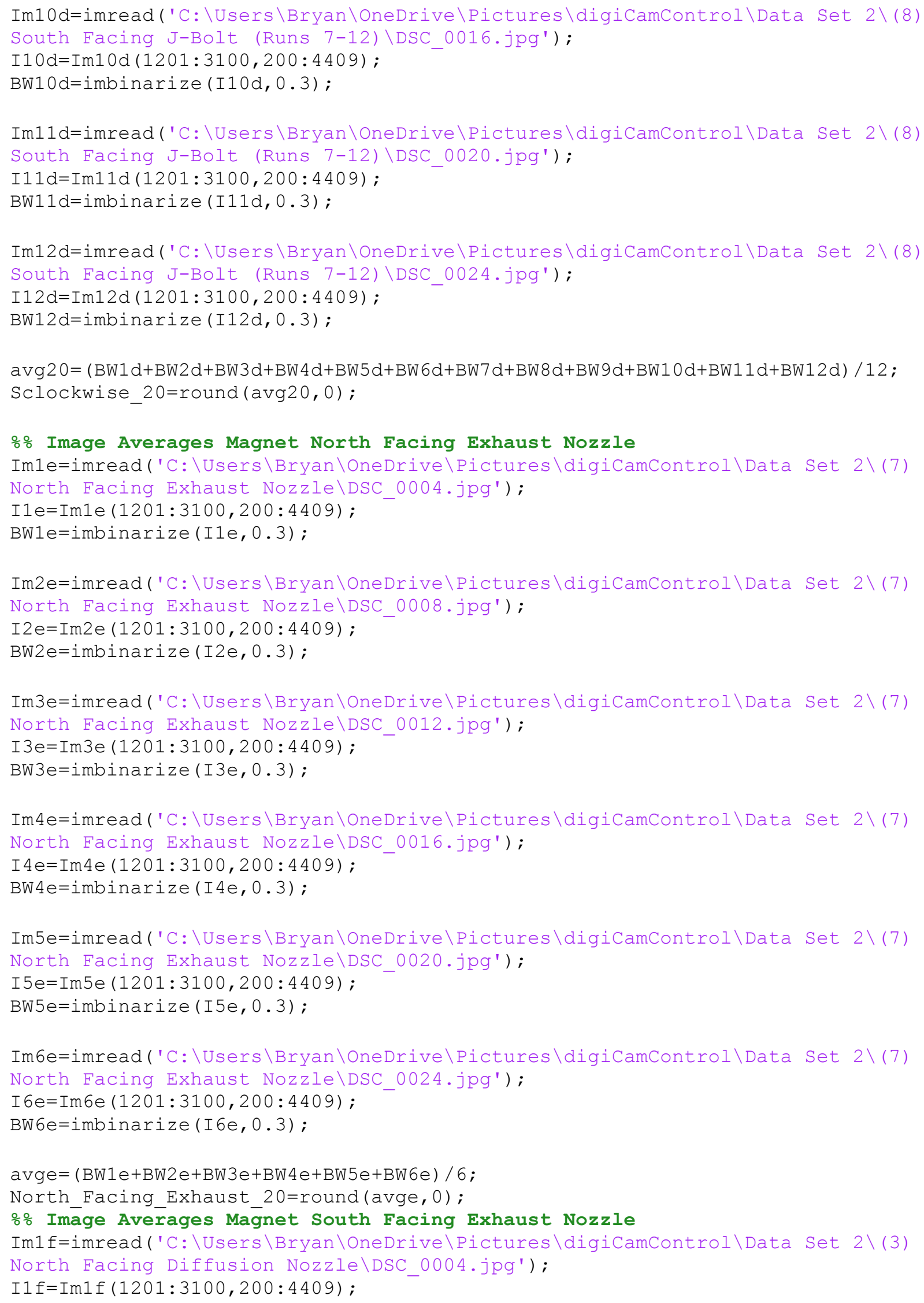




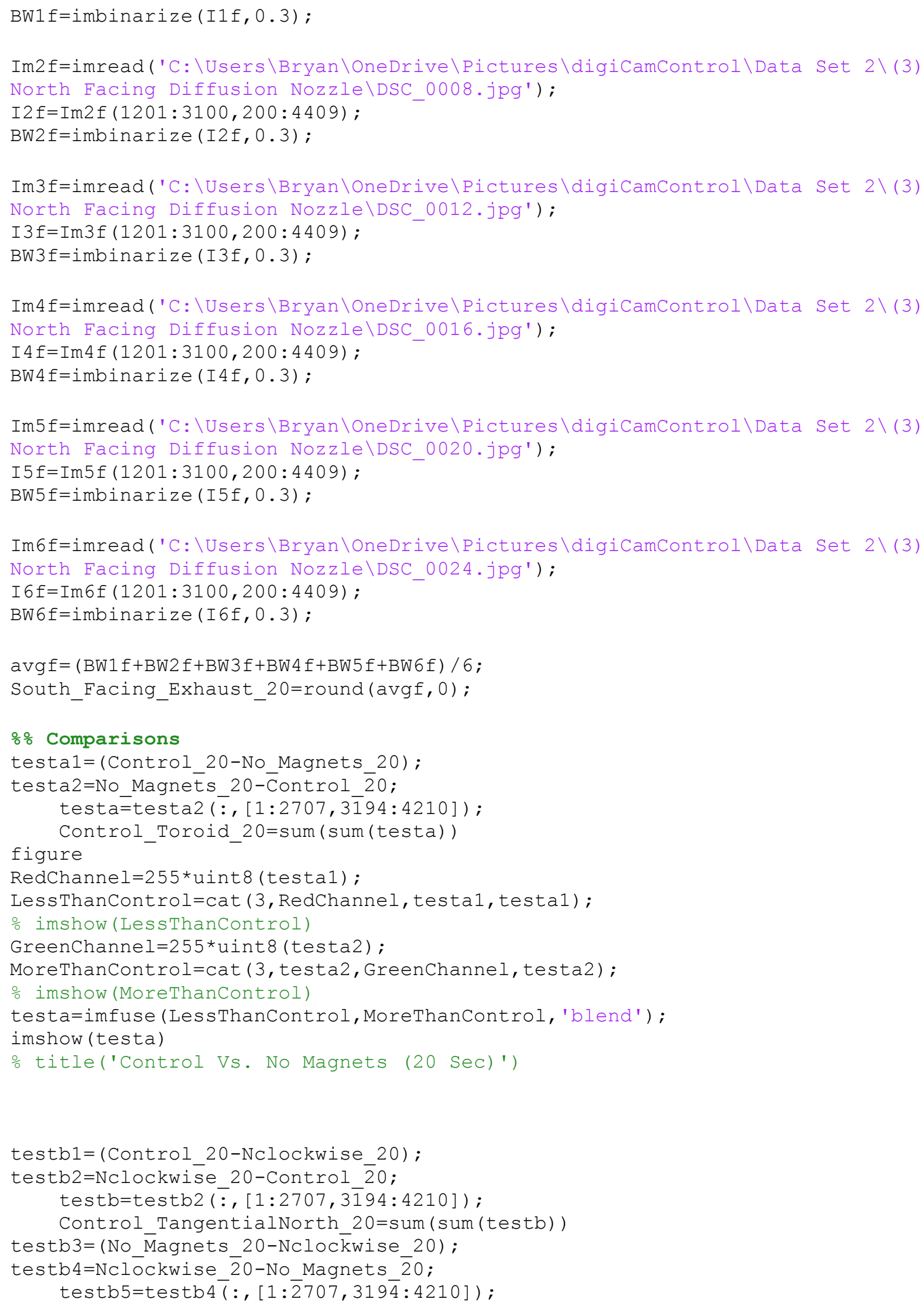




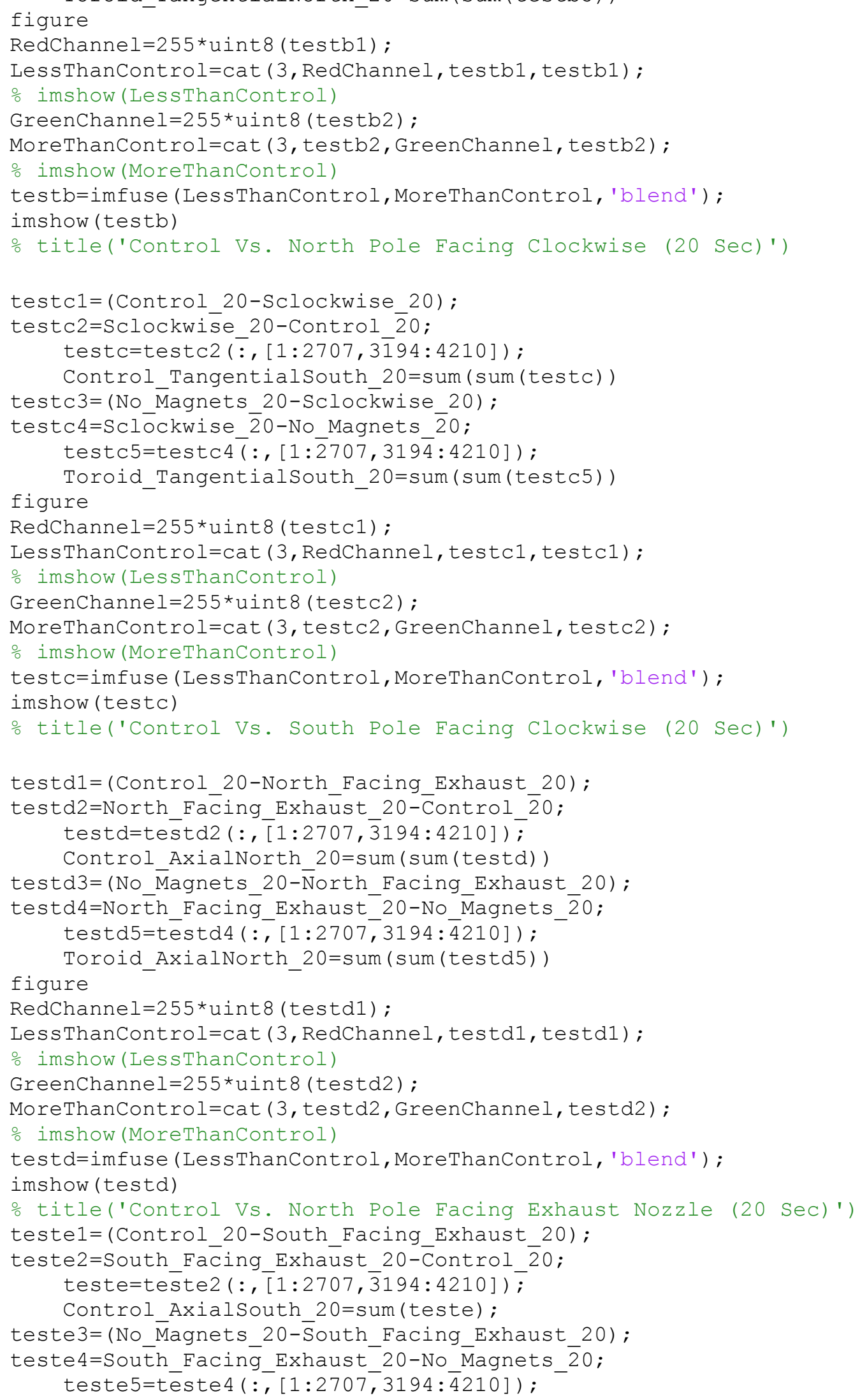


figure

Toroid_Axialsouth_20=sum (sum (teste45))

RedChannel $=255 *$ uint8 (teste1);

LessThanControl=cat (3, RedChannel, teste1, teste1) ;

\% imshow (LessThanControl)

GreenChannel $=255$ *uint 8 (teste2);

MoreThanControl=cat (3, teste2, GreenChannel, teste2) ;

\% imshow (MoreThanControl)

teste=imfuse (Less ThanControl, MoreThanControl, 'blend' ) ;

imshow (teste)

o title('Control Vs. South Pole Facing Exhaust Nozzle (20 Sec)') 


\section{Appendix F: MATLAB Code for Data Set 2 at 30 seconds}

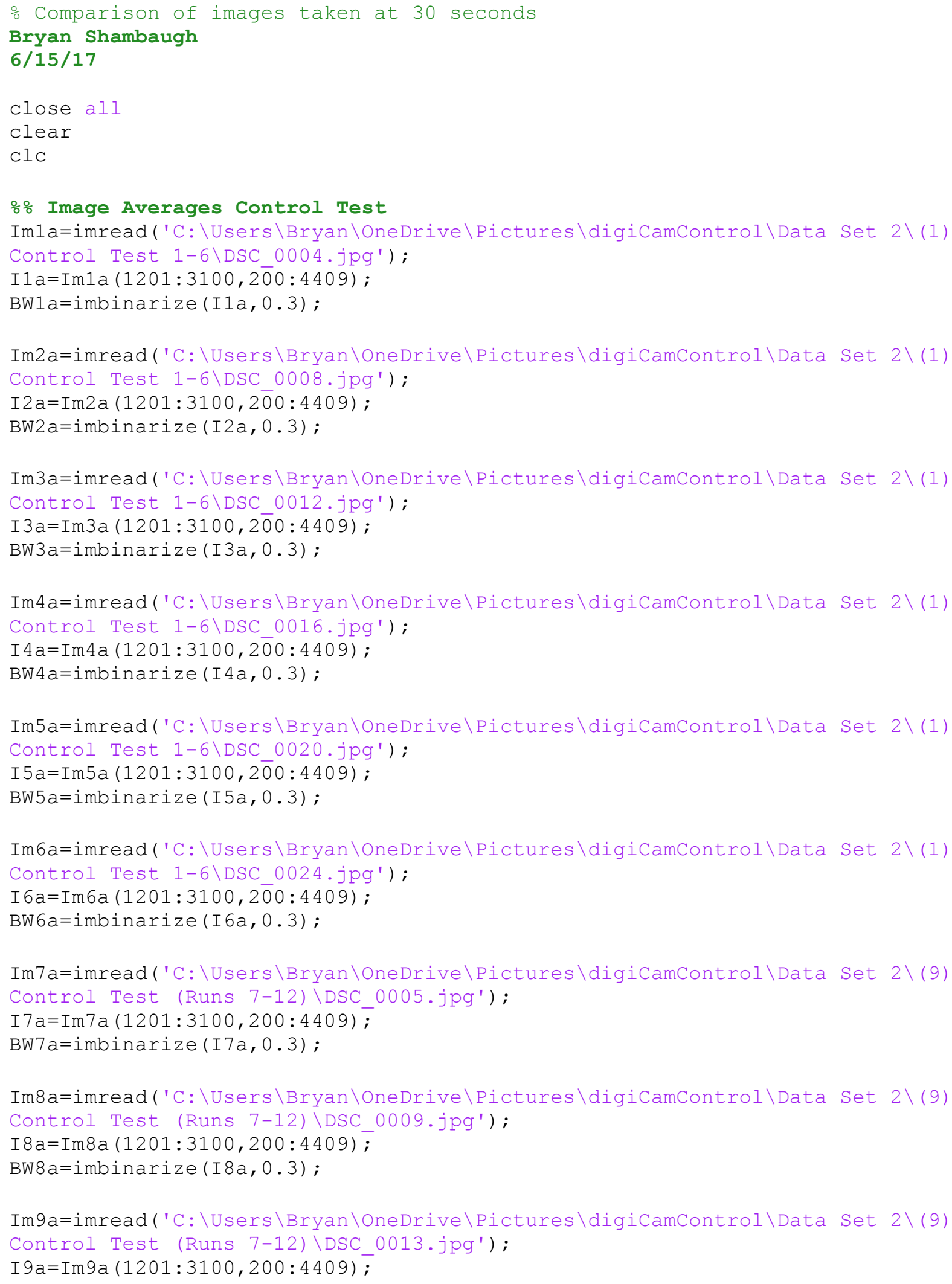




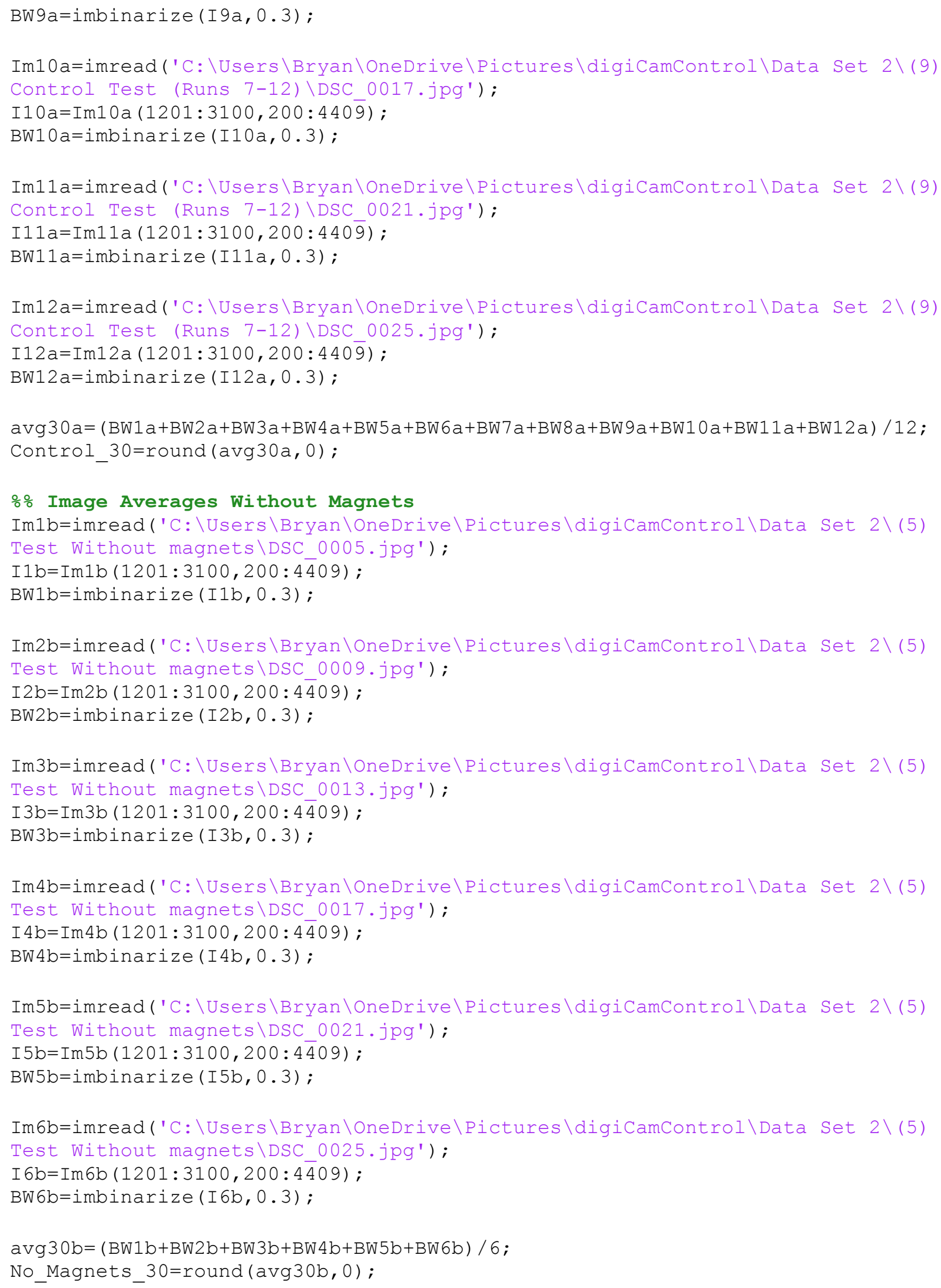




\section{응 Image Averages North Facing Clockwise}

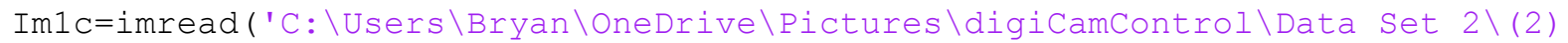
North Facing J-Bolt (Runs 1-6) \DSC_0005.jpg');

$I 1 C=\operatorname{Im} 1 \mathrm{C}(1201: 3100,200: 4409)$;

BW1 $=$ imbinarize (IIC,0.3);

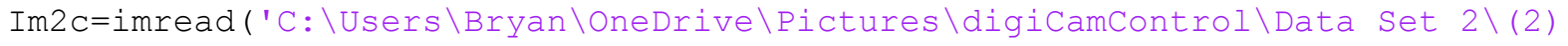
North Facing J-Bolt (Runs 1-6) \DSC_0009.jpg');

$I 2 c=\operatorname{Im} 2 c(1201: 3100,200: 4409)$;

$\mathrm{BW} 2 \mathrm{C}=$ imbinarize $(\mathrm{I} 2 \mathrm{C}, 0.3)$;

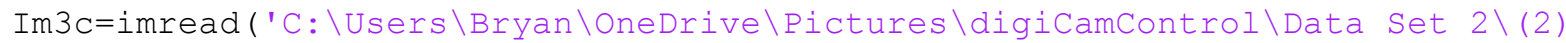
North Facing J-Bolt (Runs 1-6) \DSC_0013.jpg');

I3c $=\operatorname{Im} 3 c(1201: 3100,200: 4409)$;

$\mathrm{BW} 3 \mathrm{C}=$ imbinarize $(\mathrm{I} 3 \mathrm{C}, 0.3)$;

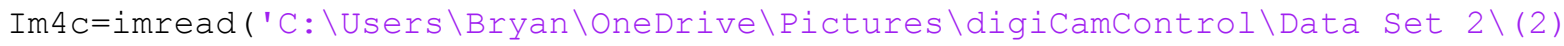
North Facing J-Bolt (Runs 1-6) \DSC_0017.jpg');

I $4 \mathrm{C}=\operatorname{Im} 4 \mathrm{C}(1201: 3100,200: 4409)$;

BW4 $=$ imbinarize ( I 4 , 0.3$)$;

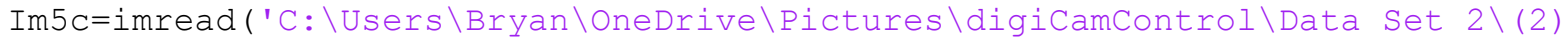
North Facing J-Bolt (Runs 1-6) \DSC_0021.jpg');

$I 5 c=\operatorname{Im} 5 c(1201: 3100,200: 4409) ;$

BW5 C=imbinarize ( I $5 \mathrm{c}, 0.3)$;

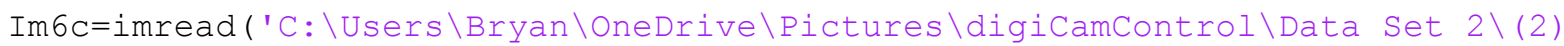
North Facing J-Bolt (Runs 1-6) \DSC 0025.jpg');

I6c $=\operatorname{Im} 6 \mathrm{c}(1201: 3100,200: 4409)$;

$\mathrm{BW} 6 \mathrm{C}=$ imbinarize $(\mathrm{I} 6 \mathrm{c}, 0.3)$;

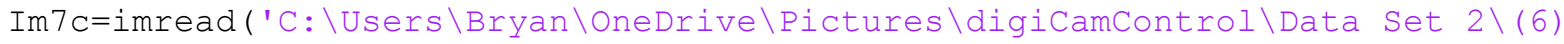
North Facing J-Bolt (Runs 7-12) \DSC_0006.jpg');

I7C $=\operatorname{Im} 7 \mathrm{C}(1201: 3100,200: 4409)$;

$\mathrm{BW} 7 \mathrm{C}=$ imbinarize $(\mathrm{I} 7 \mathrm{C}, 0.3)$;

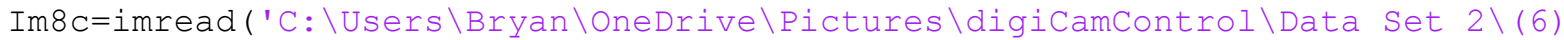
North Facing J-Bolt (Runs 7-12) \DSC 0010.jpg');

$I 8 c=\operatorname{Im} 8 \mathrm{C}(1201: 3100,200: 4409) ;$

$\mathrm{BW} 8 \mathrm{c}=$ imbinarize $(\mathrm{I} 8 \mathrm{c}, 0.3)$;

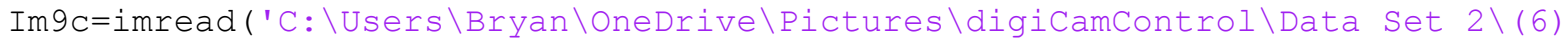
North Facing J-Bolt (Runs 7-12) \DSC_0014.jpg');

$I 9 C=\operatorname{Im} 9 c(1201: 3100,200: 4409)$;

BW9C=imbinarize (I9c,0.3);

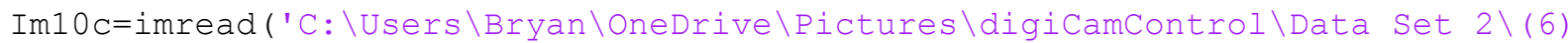
North Facing J-Bolt (Runs 7-12) \DSC_0018.jpg');

$\operatorname{I10C}=\operatorname{Im10c}(1201: 3100,200: 4409)$;

BW10c=imbinarize $(\mathrm{I} 10 \mathrm{c}, 0.3)$;

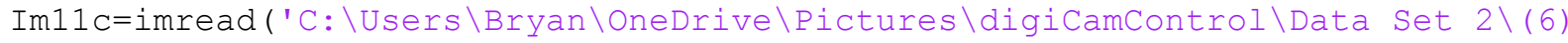
North Facing J-Bolt (Runs 7-12) \DSC_0022.jpg');

I11C $=\operatorname{Im11C}(1201: 3100,200: 4409)$;

BW11 $=$ imbinarize $(111 \mathrm{c}, 0.3)$; 


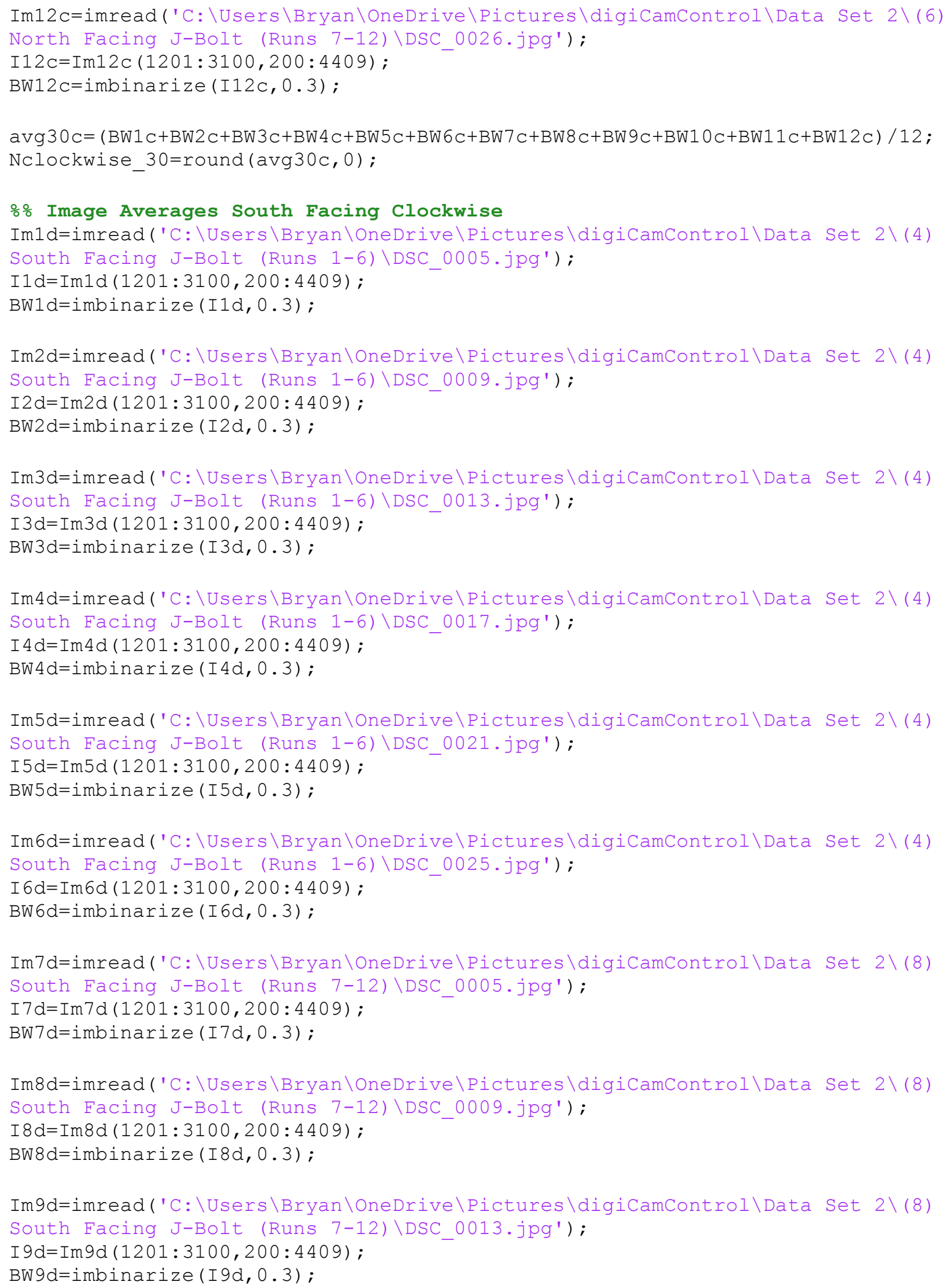




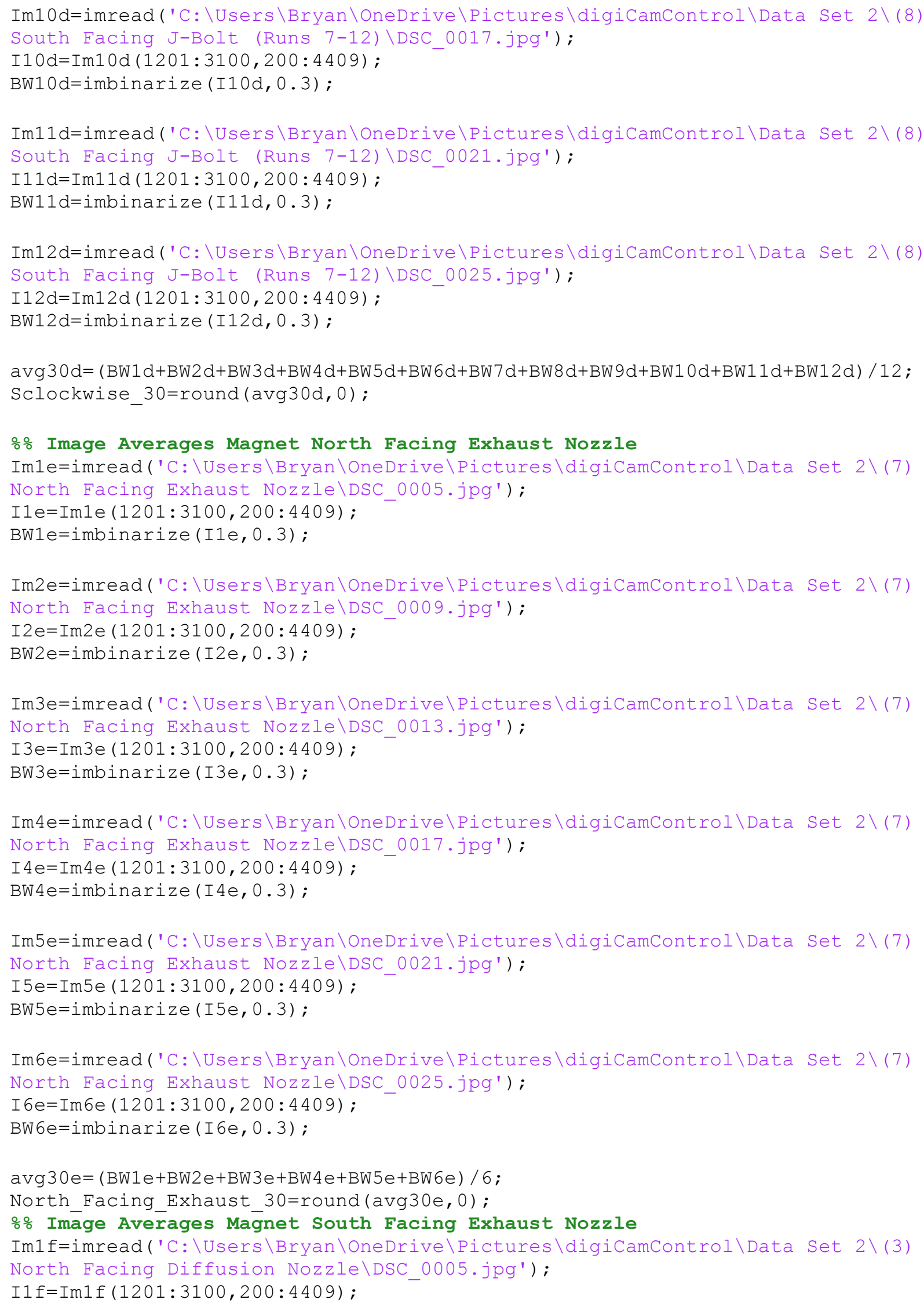




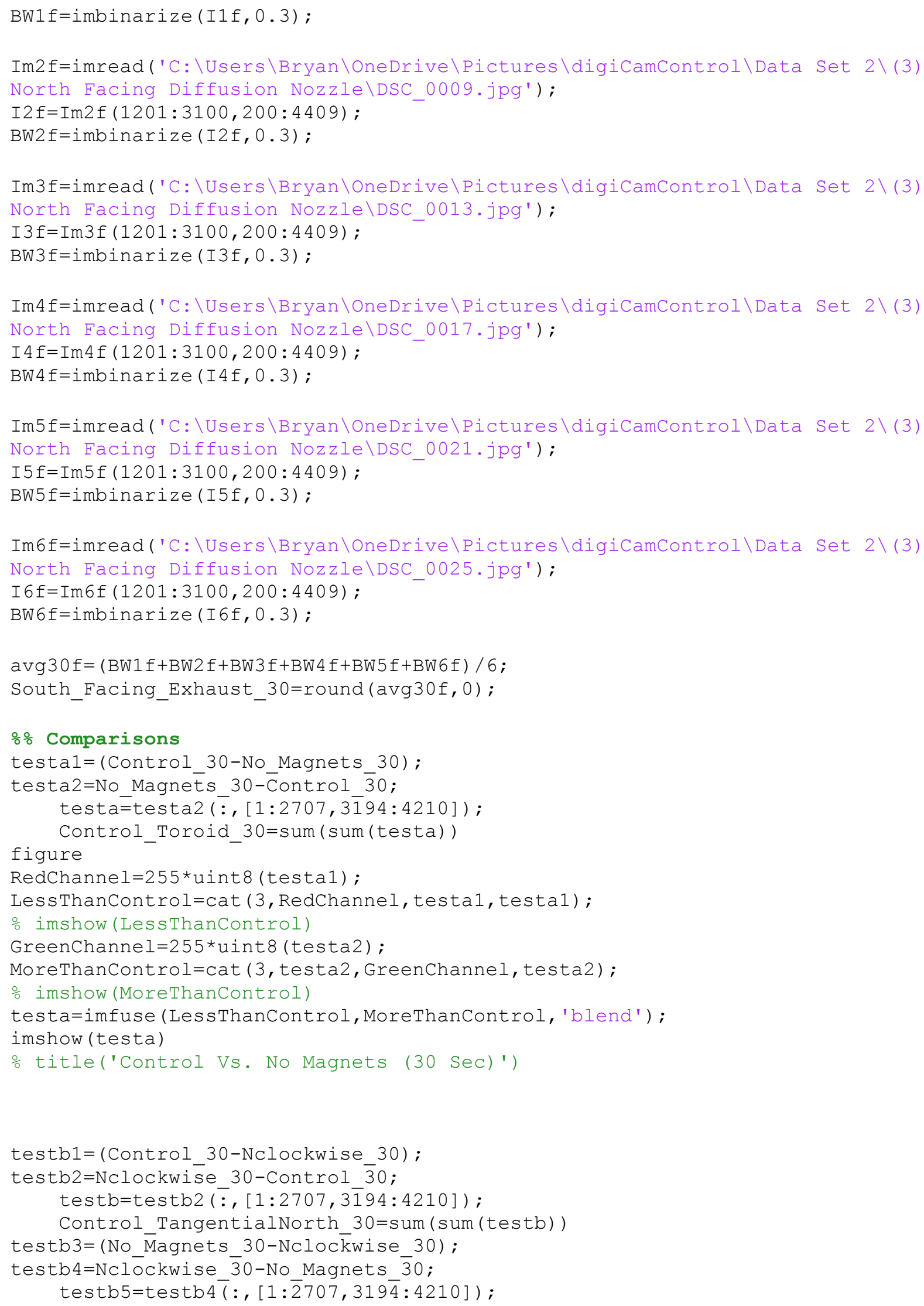




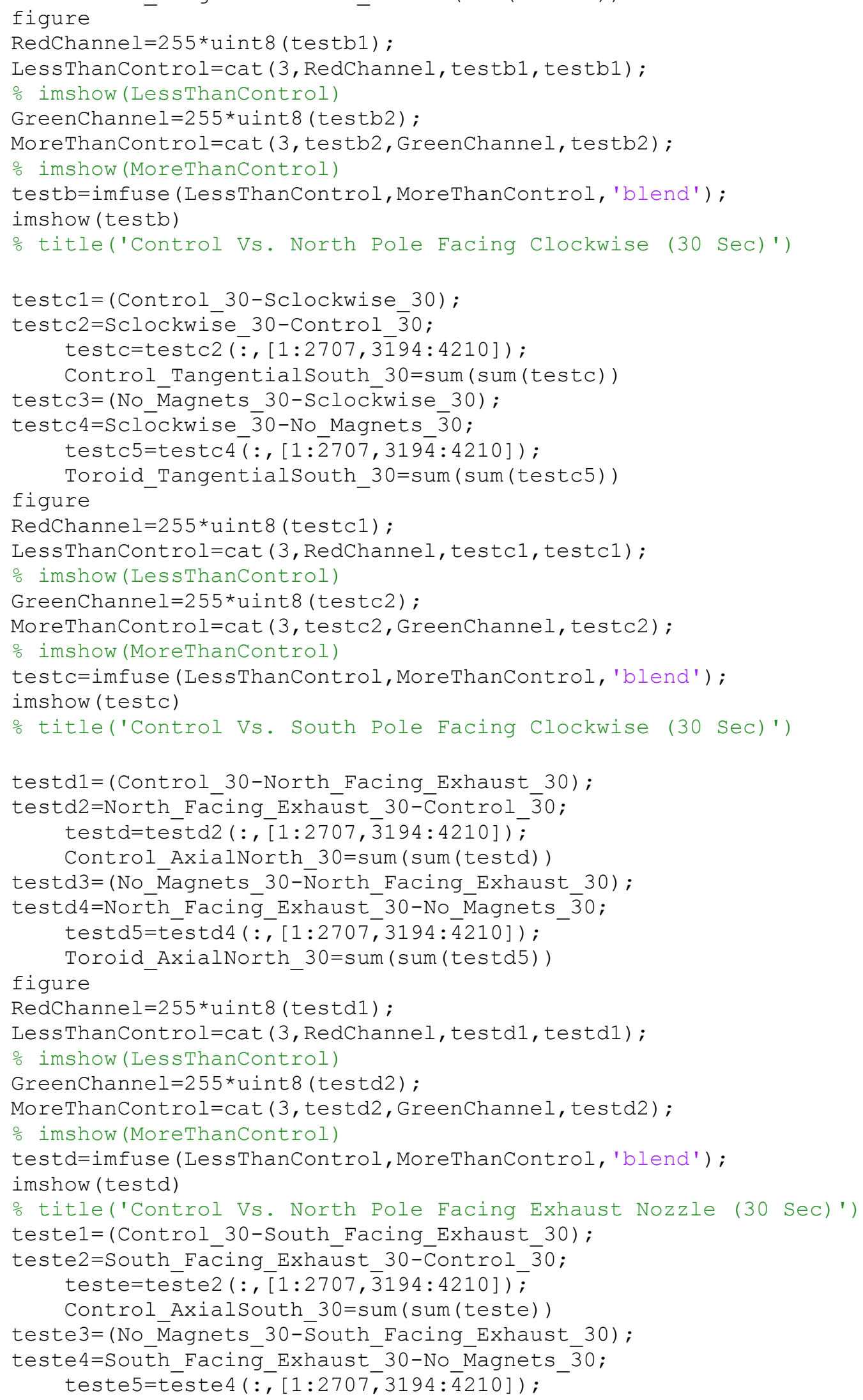


figure

Toroid_AxialSouth_30=sum (sum(teste5))

RedChannel $=255 *$ uint8 (teste1);

LessThanControl=cat (3, RedChannel, teste1, teste1) ;

\% imshow (LessThanControl)

GreenChannel $=255$ *uint 8 (teste2);

MoreThanControl=cat (3, teste2, GreenChannel, teste2) ;

\% imshow (MoreThanControl)

teste=imfuse (LessThanControl, MoreThanControl, ' blend ' ) ;

imshow (teste)

\% title('Control Vs. South Pole Facing Exhaust Nozzle (30 Sec)') 


\section{Appendix G: Data Set 2 - Comparison to Control Test}

Figure 42: Control vs. toroid test (10 sec.)

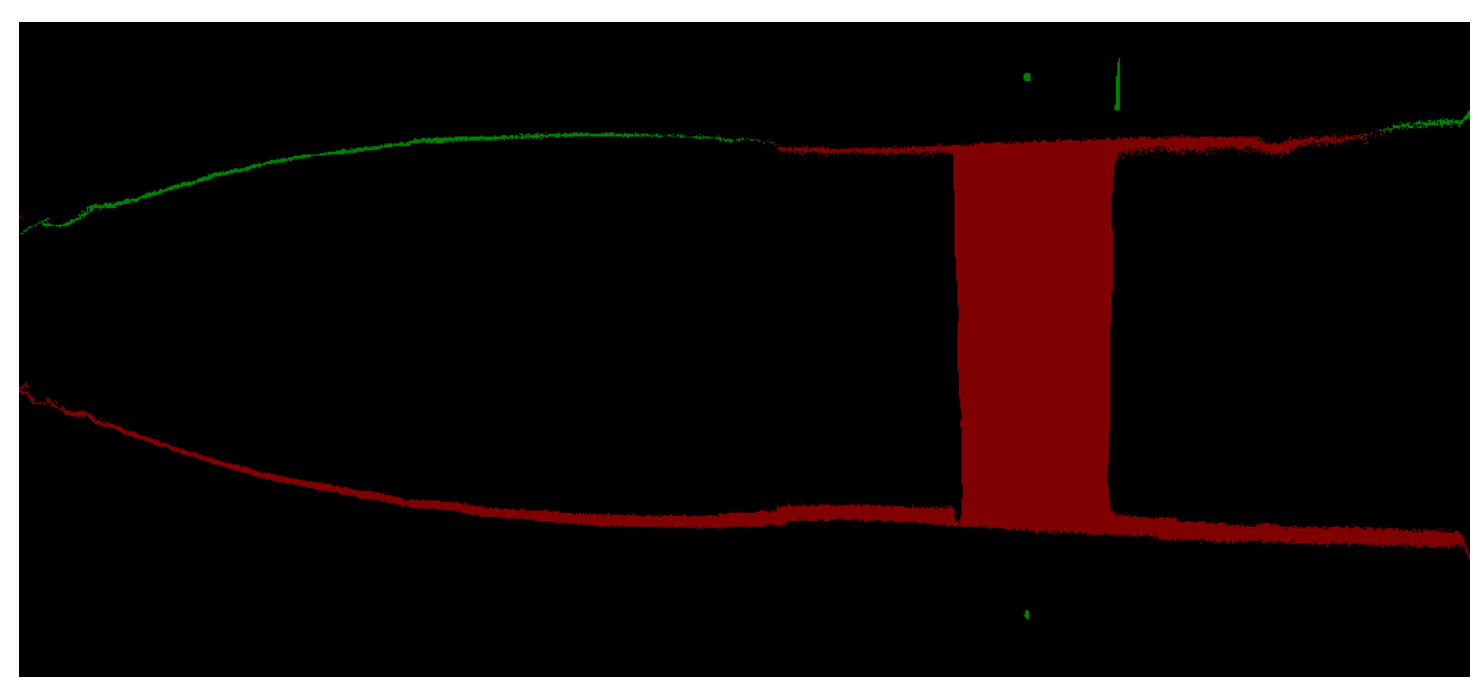

Figure 43: Control vs. toroid test (20 sec.)

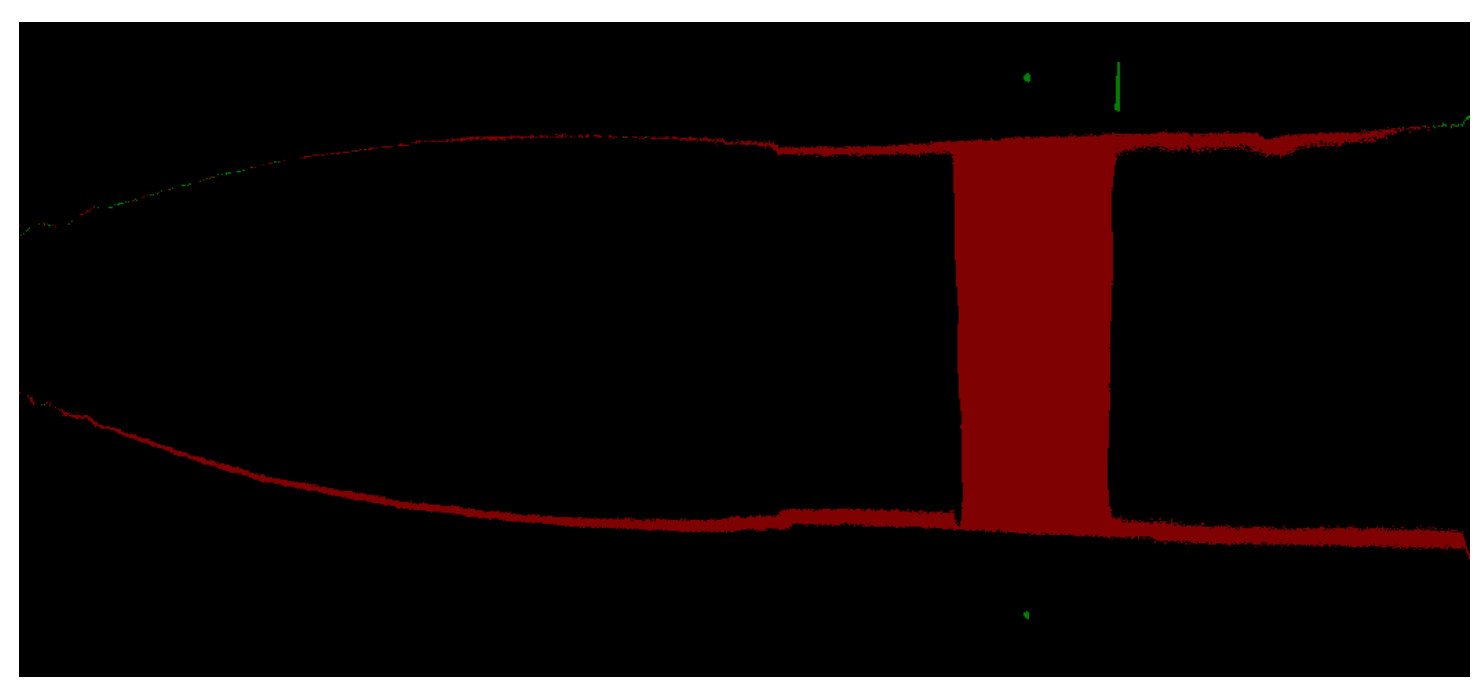

Figure 44: Control vs. toroid test (30 sec.) 


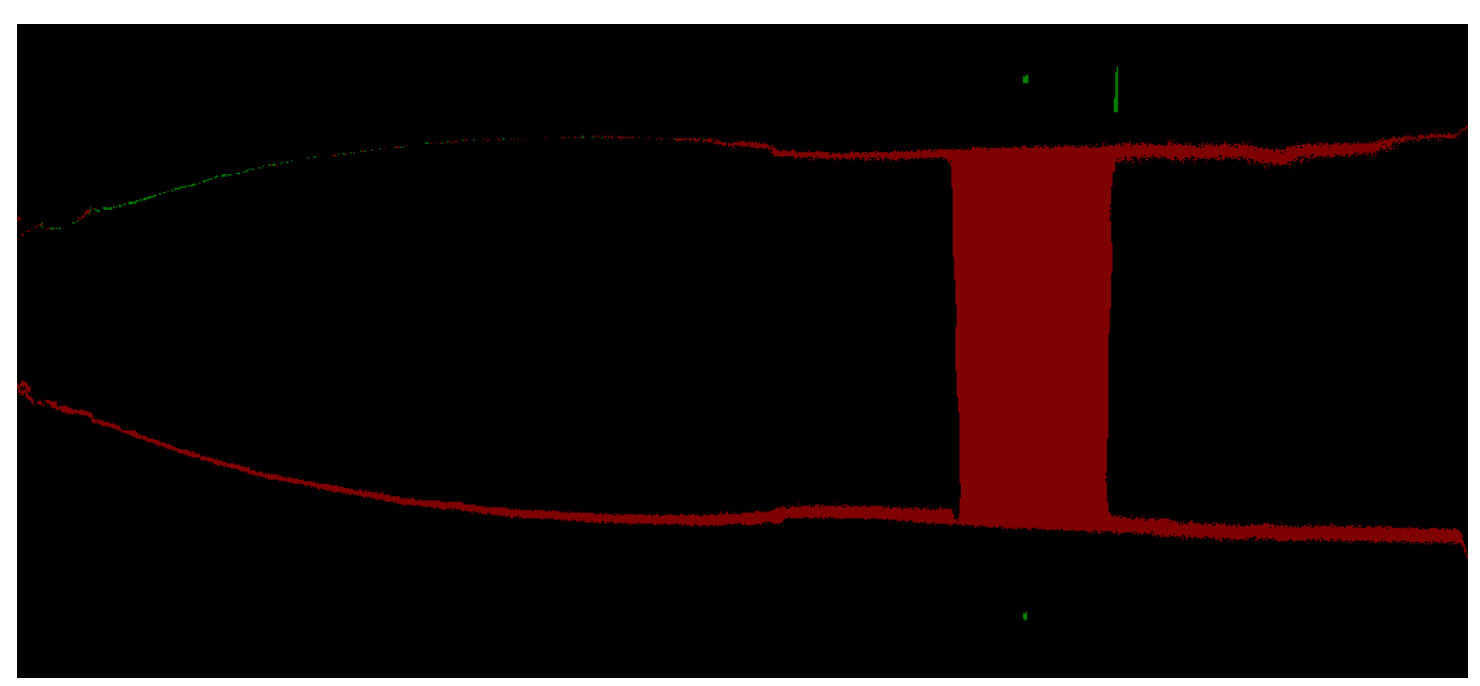

Figure 45: Control vs. tangential north test (10 sec.)

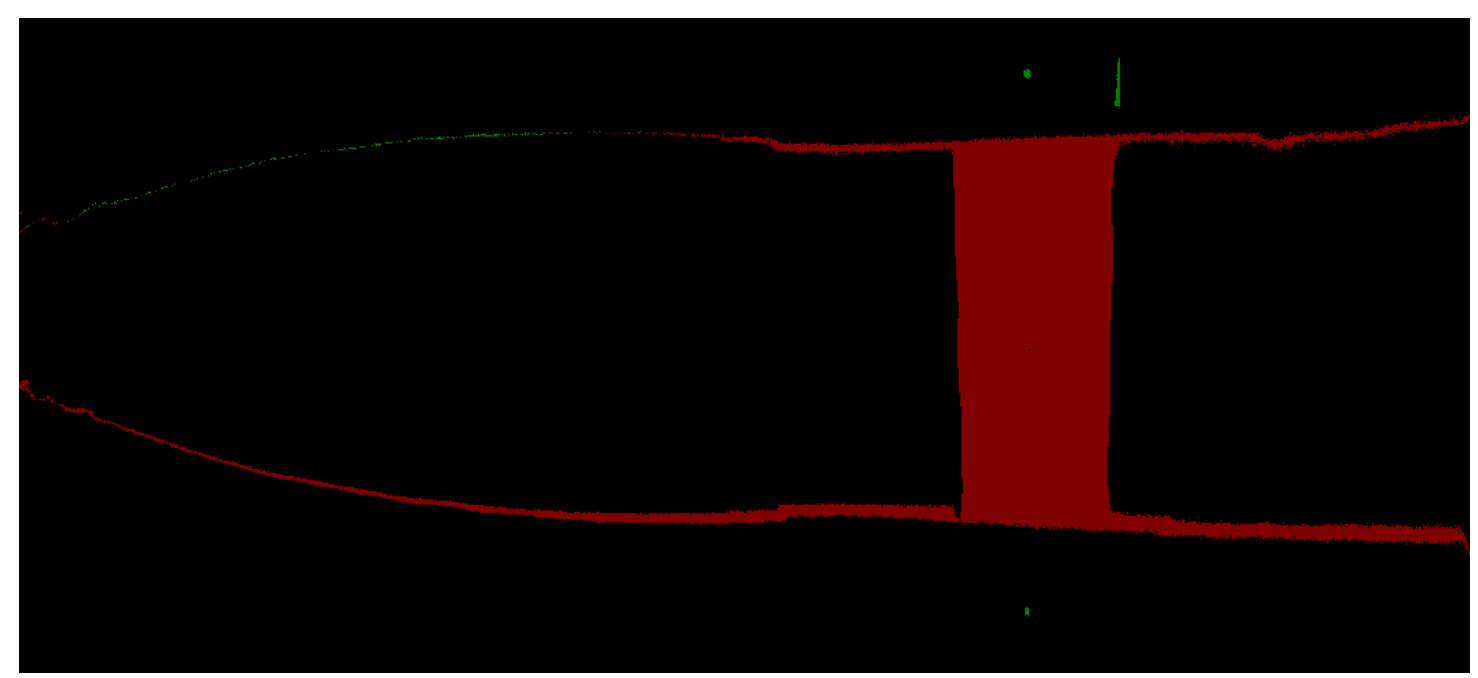

Figure 46: Control vs. tangential north test (20 sec.)

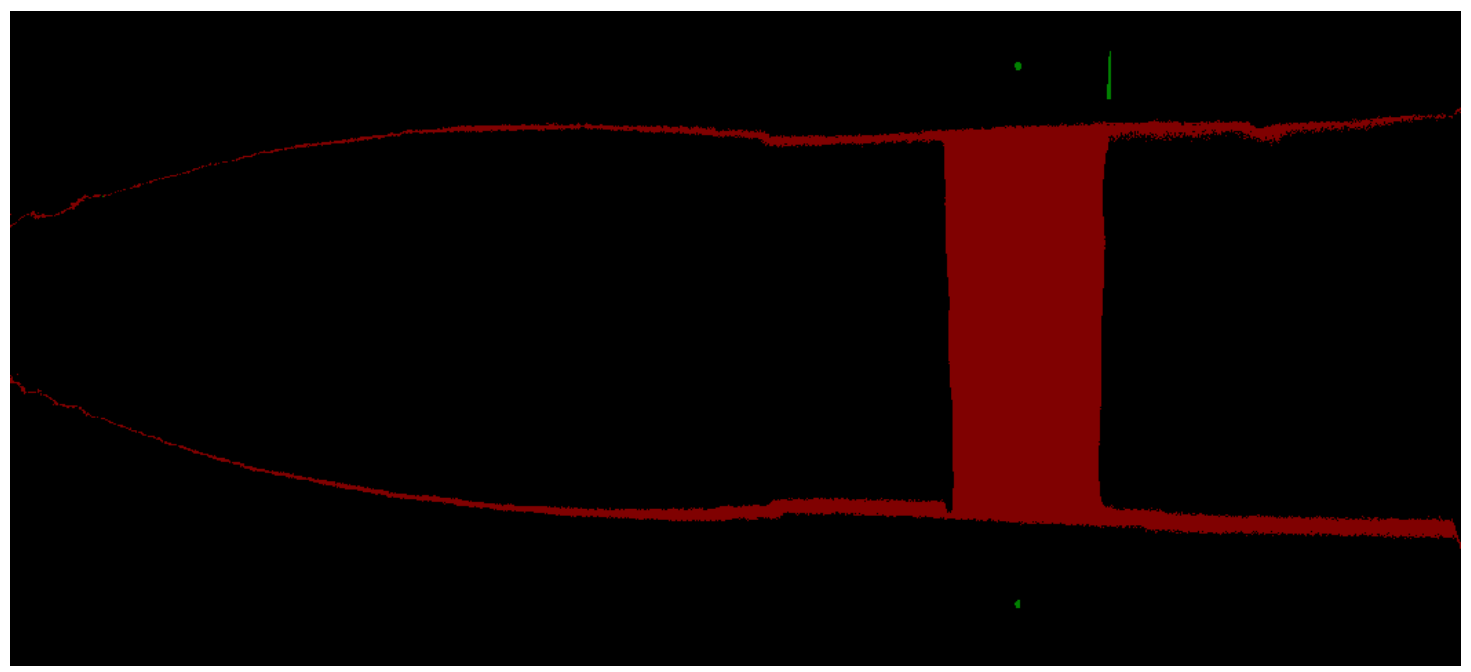

Figure 47: Control vs. tangential north test (30 sec.) 


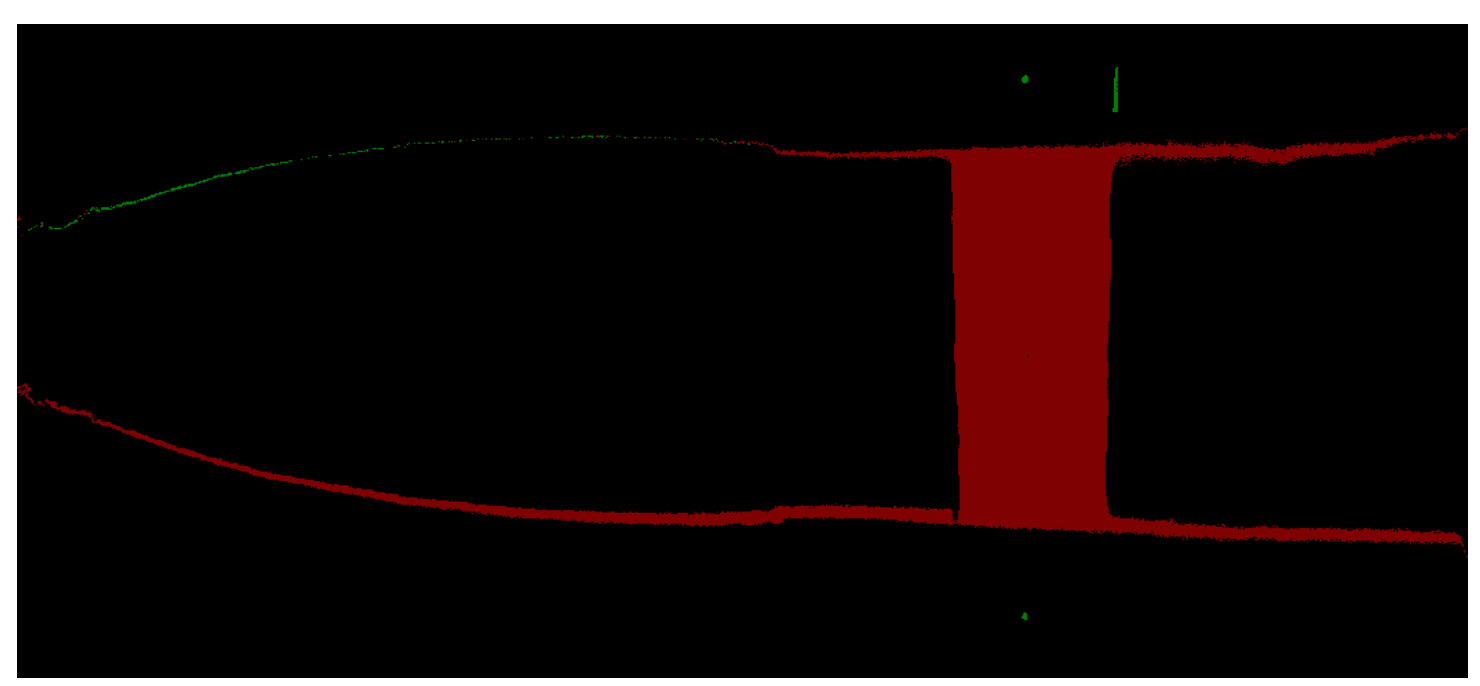

Figure 48: Control vs. tangential south test (10 sec.)

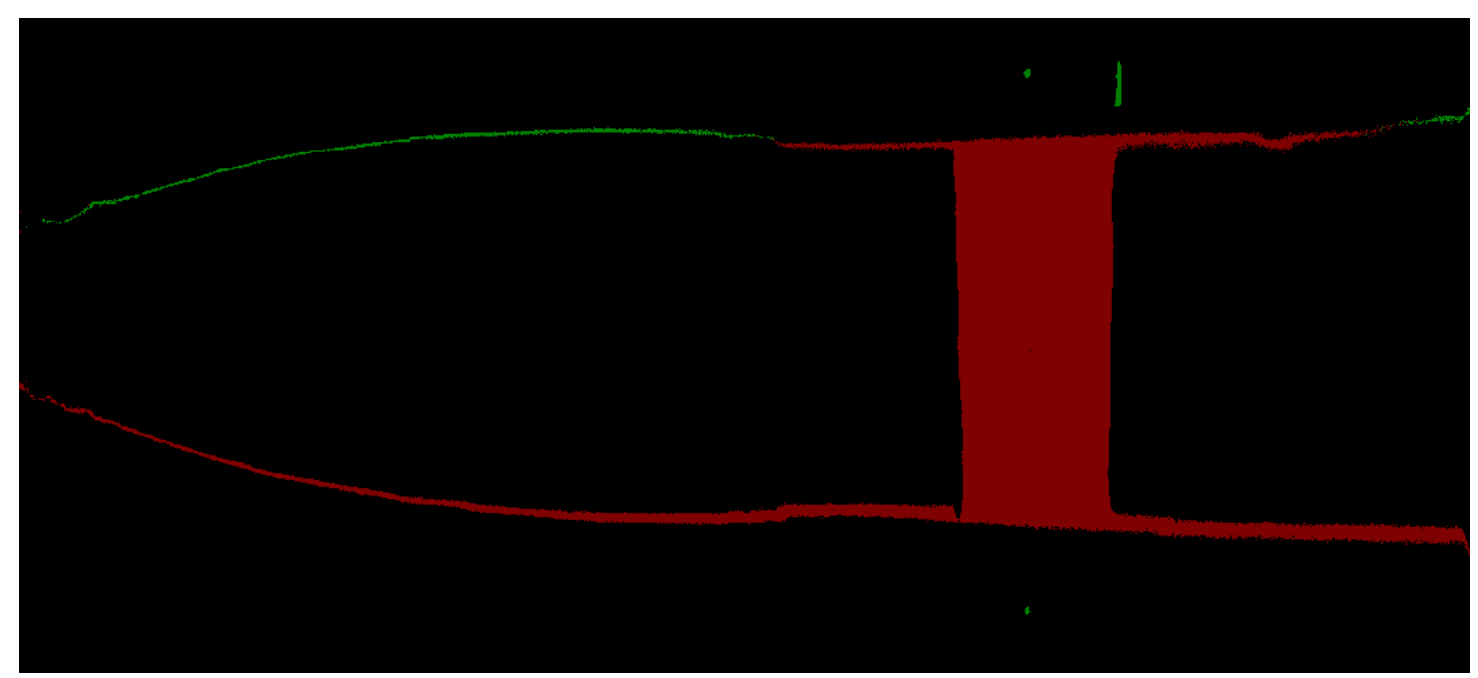

Figure 49: Control vs. tangential south test (20 sec.)

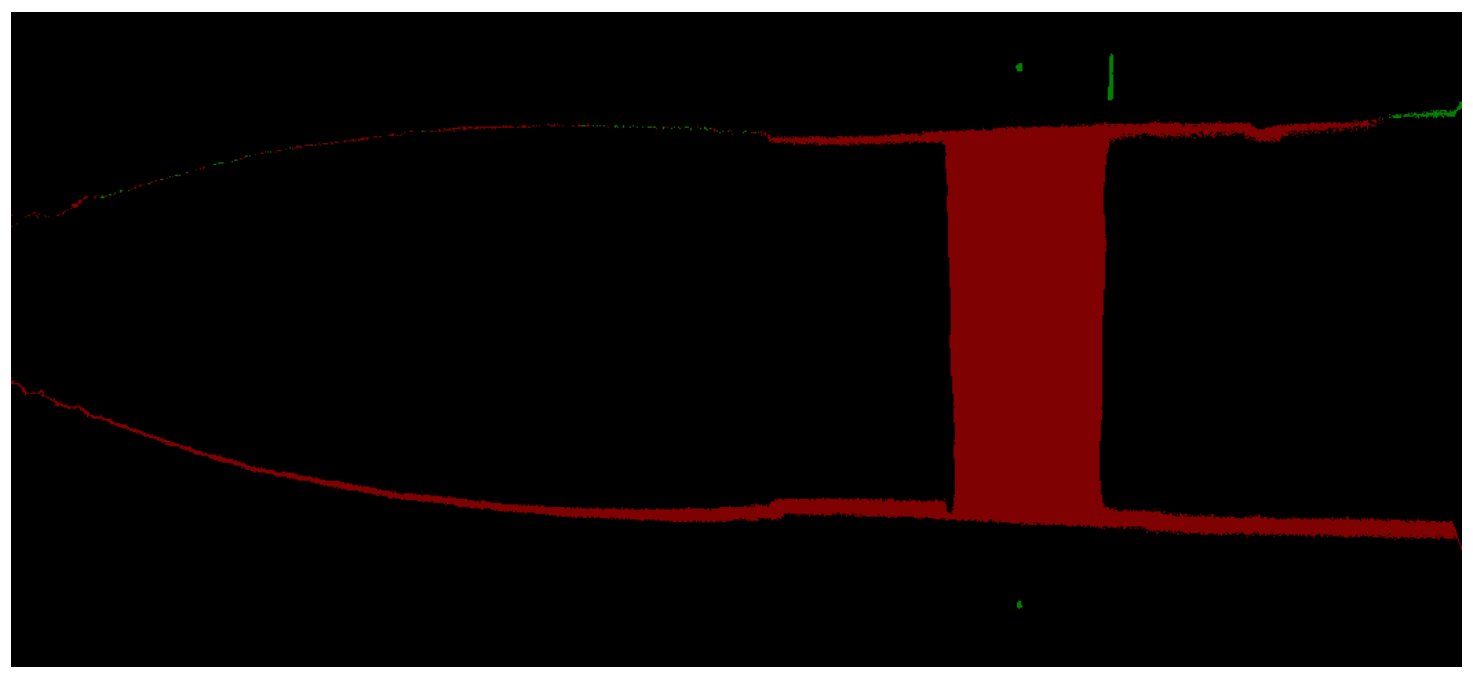

Figure 50: Control vs. tangential south test (30 sec.) 


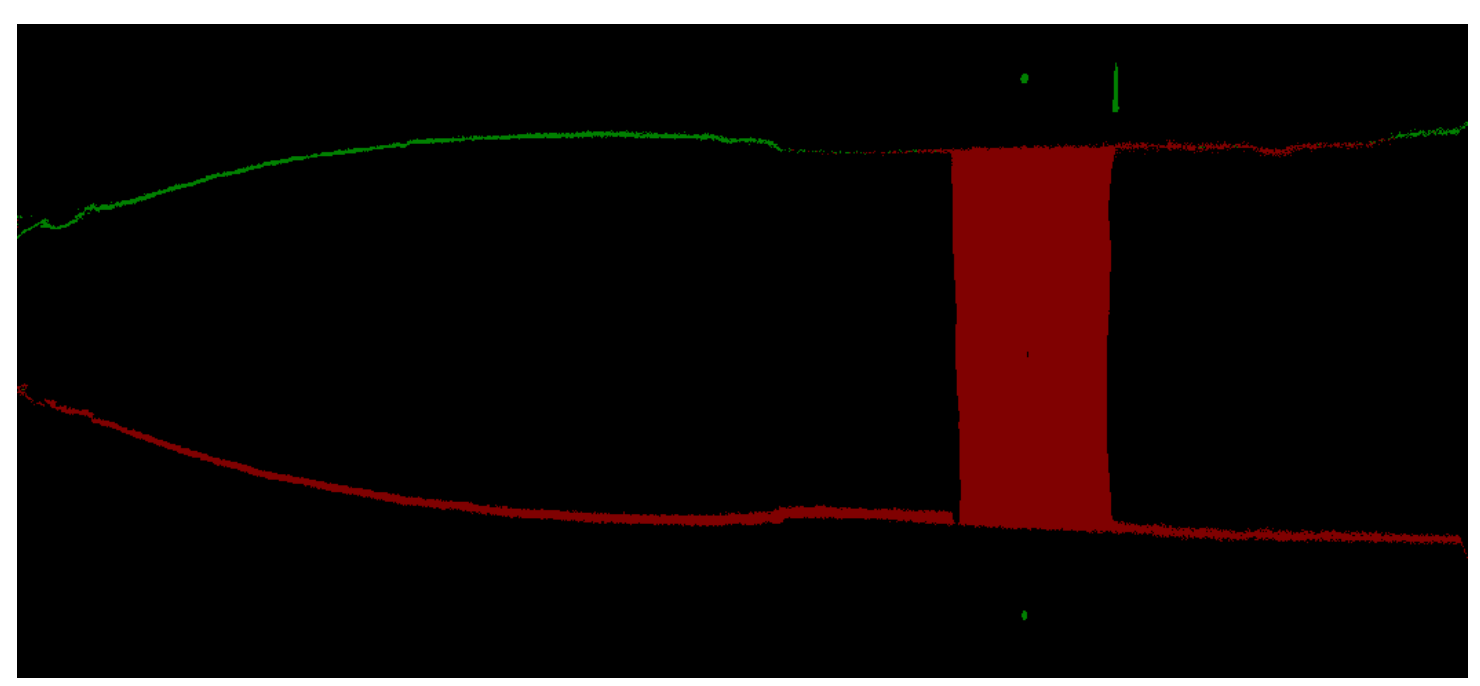

Figure 51: Control vs. axial test (10 sec.)

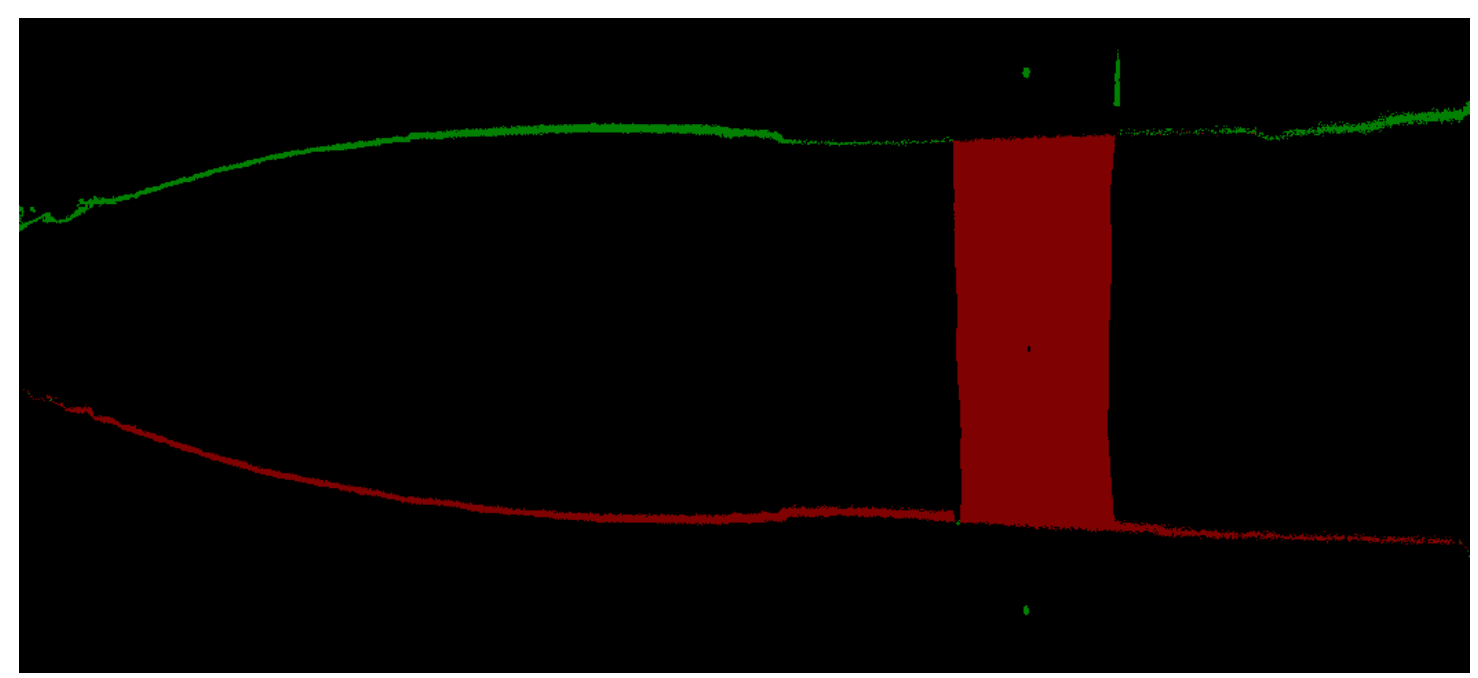

Figure 52: Control vs. axial test (20 sec.)

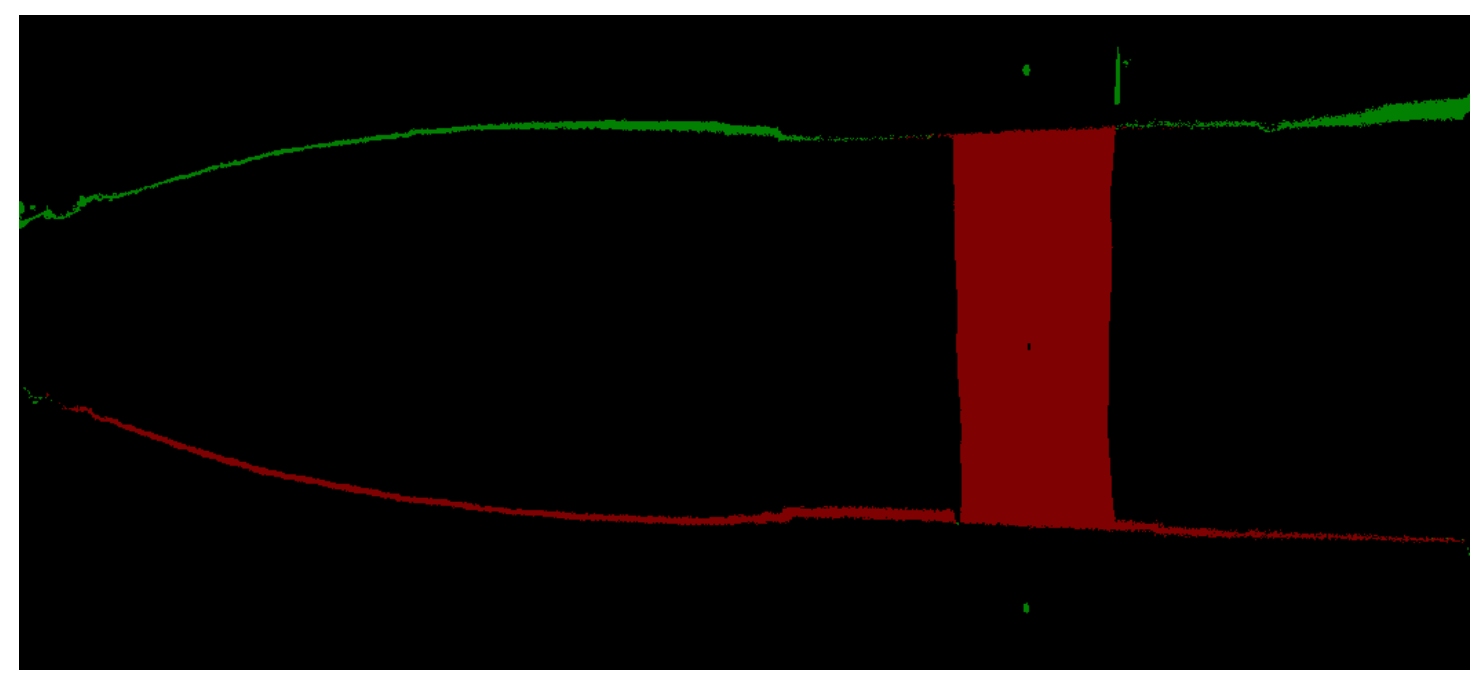

Figure 53: Control vs. axial test (30 sec.) 


\section{Appendix H: Data Set 2-Comparison to Toroid Test}

Figure 54: Toroid test vs. tangential north test (10 sec.)

Figure 55: Toroid test vs. tangential north test (20 sec.)

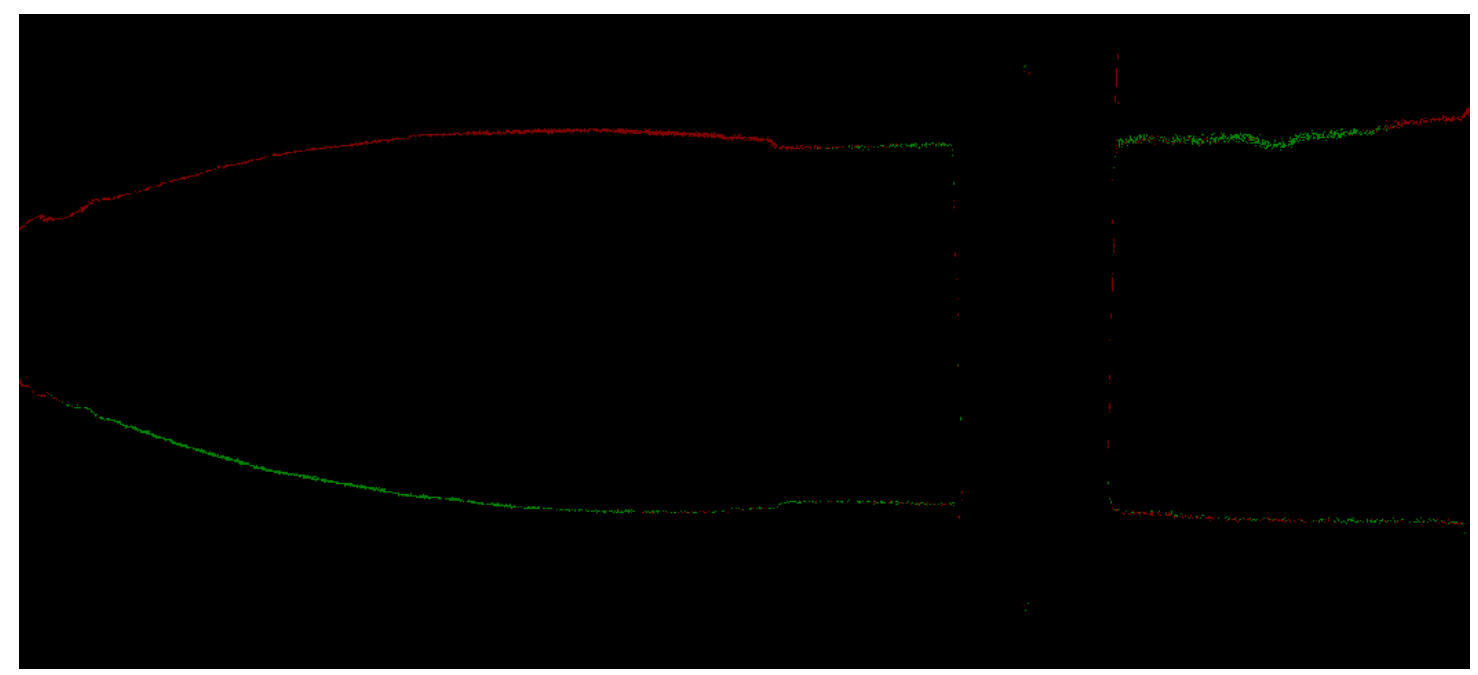

Figure 56: Toroid test vs. tangential north test (30 sec.) 


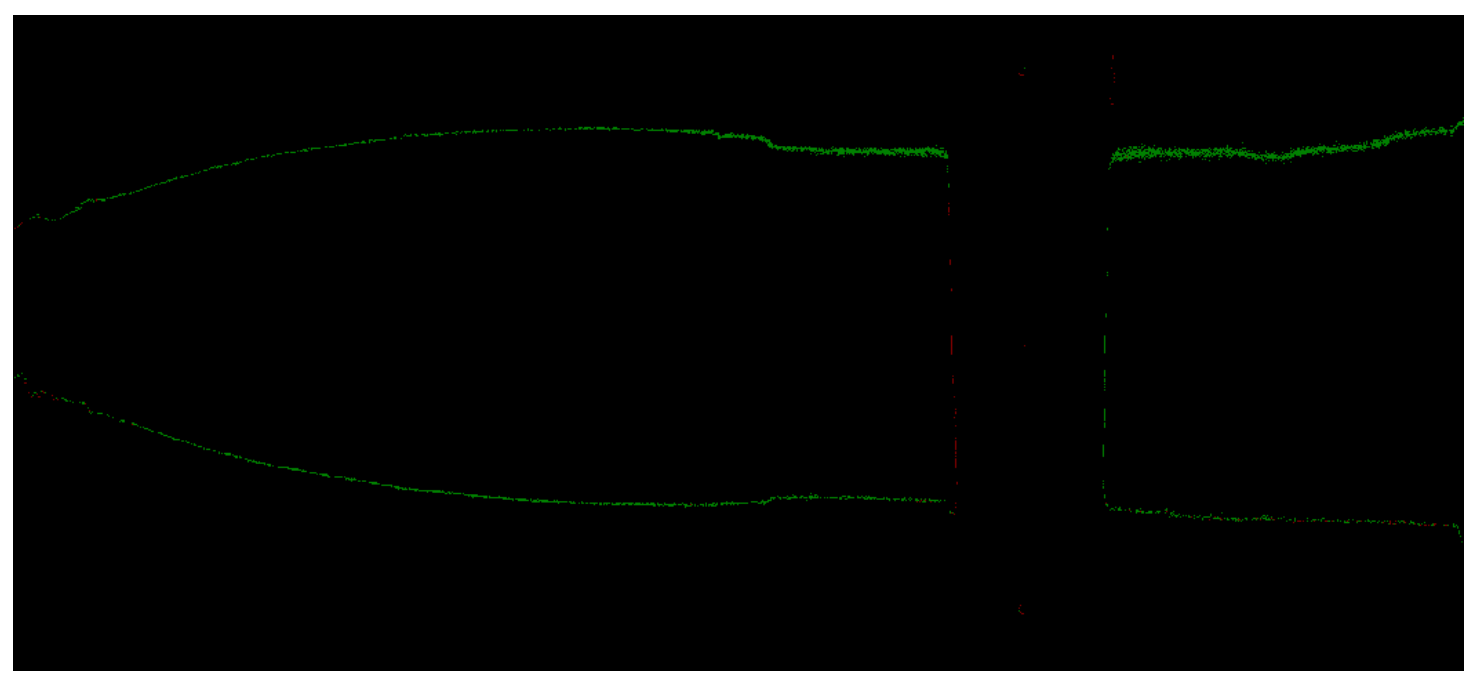

Figure 57: Toroid test vs. tangential south test (10 sec.)

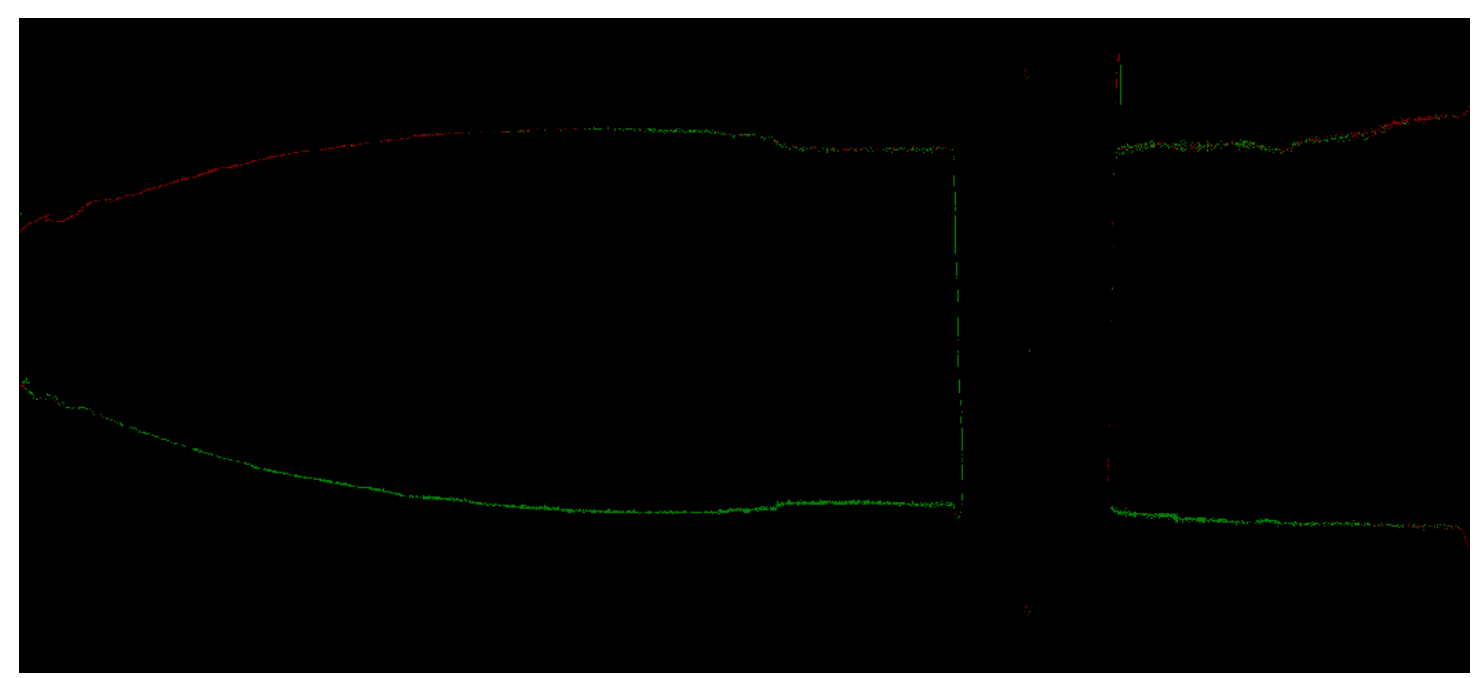

Figure 58: Toroid test vs. tangential south test (20 sec.)

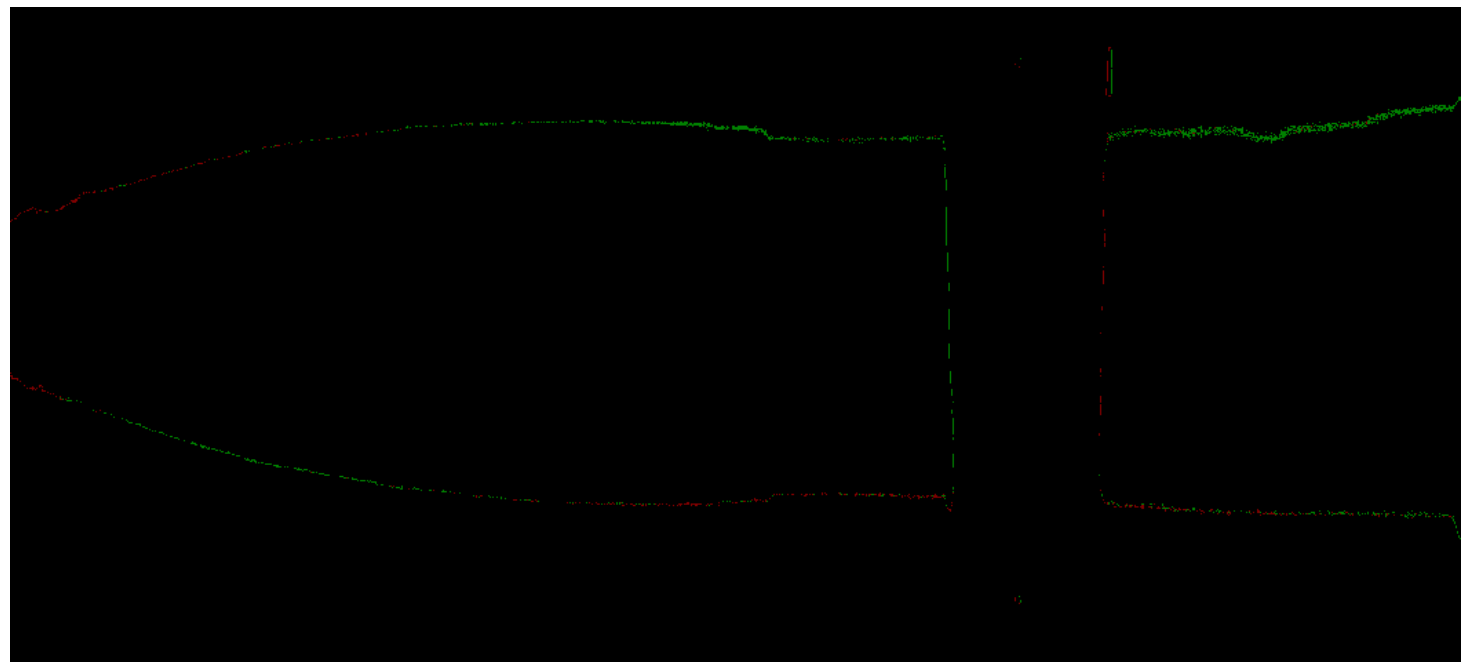

Figure 59: Toroid test vs. tangential south test ( $30 \mathrm{sec}$. 


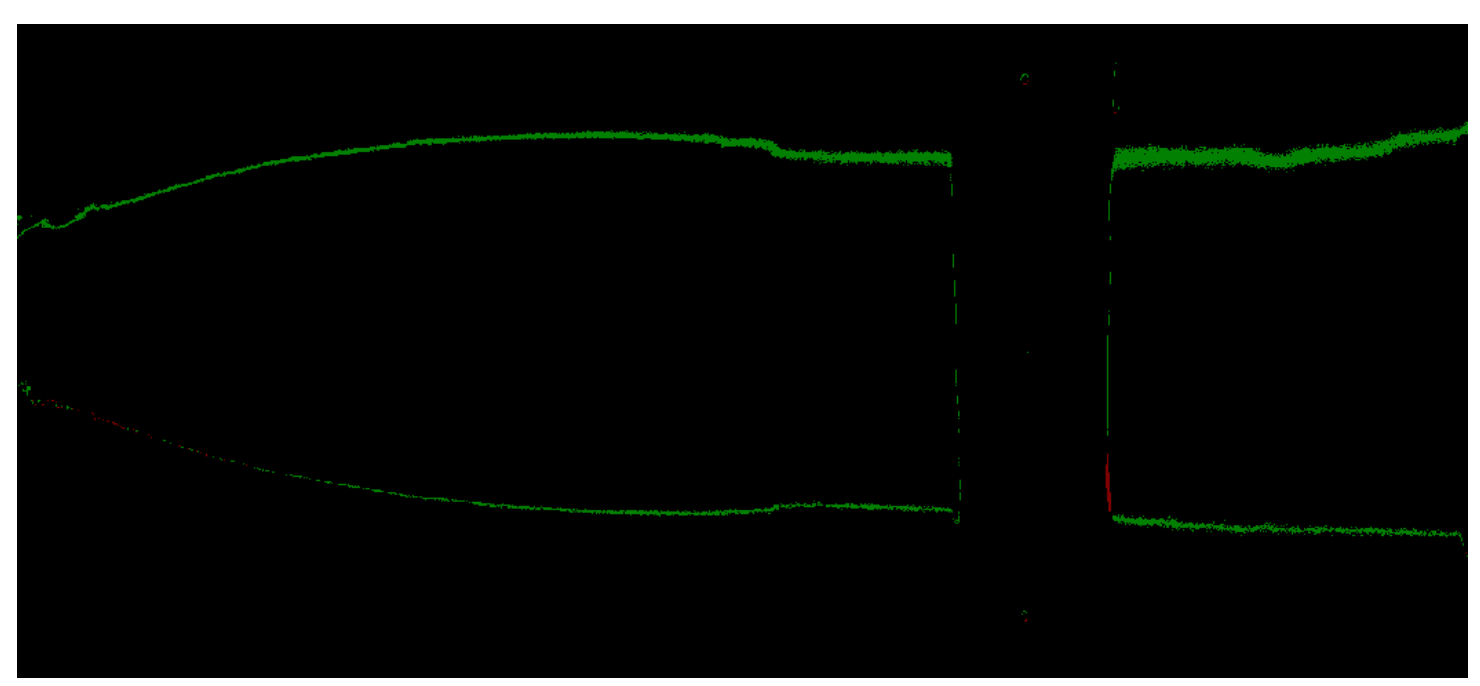

Figure 60: Toroid test vs. axial test (10 sec.)

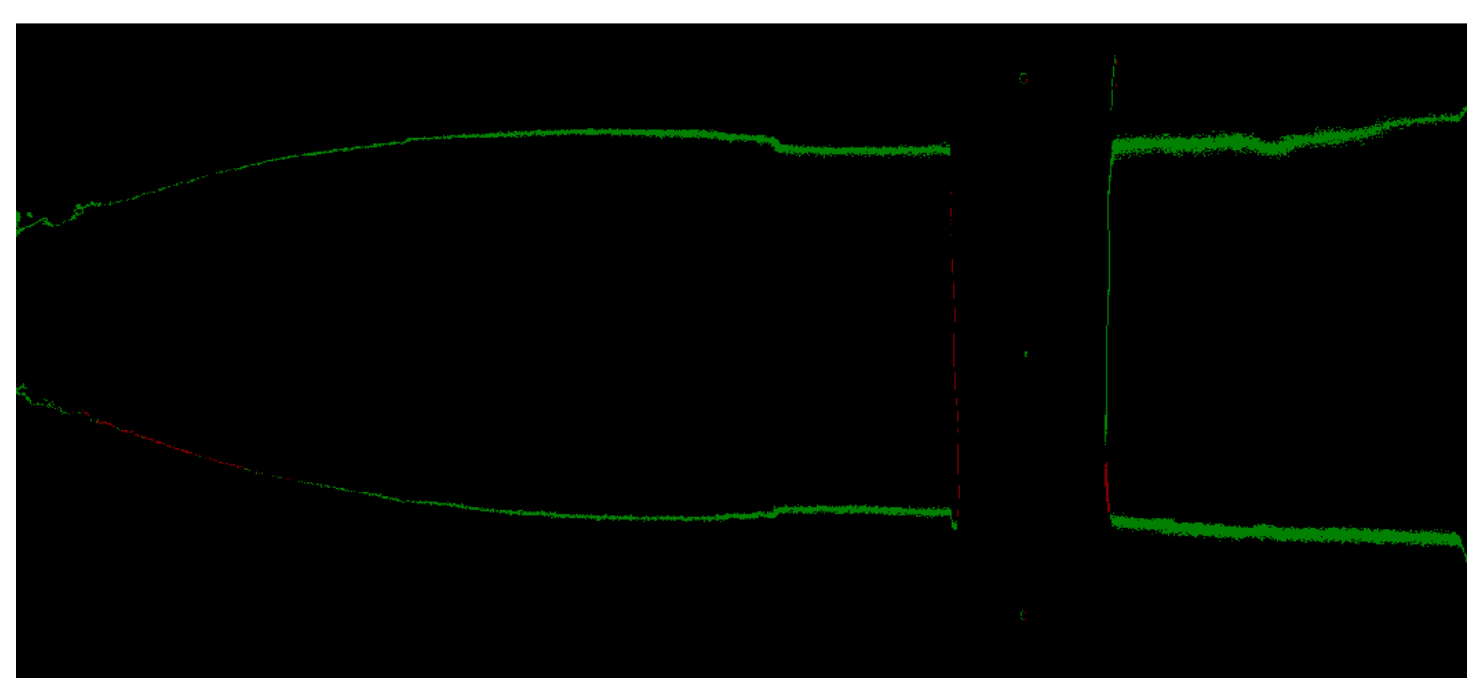

Figure 61: Toroid test vs. axial test (20 sec.)

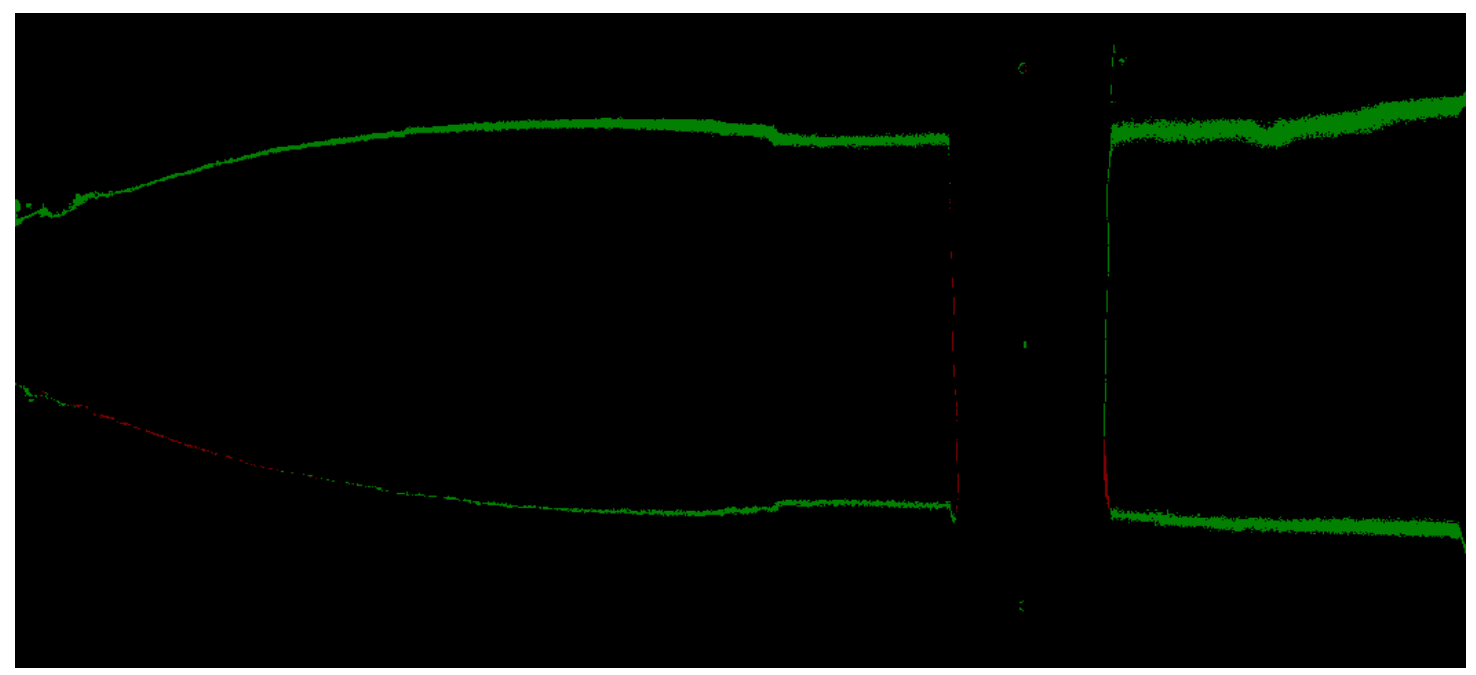

Figure 62: Toroid test vs. axial test (30 sec.) 